ITP-UH-22/14

EMPG-14-20

\title{
Sasakian quiver gauge theories and instantons on Calabi-Yau cones
}

\author{
Olaf Lechtenfeld ${ }^{1}$, Alexander D. Popov ${ }^{1}$ and Richard J. Szabo ${ }^{2}$ \\ ${ }^{1}$ Institut für Theoretische Physik and Riemann Center for Geometry and Physics \\ Leibniz Universität Hannover \\ Appelstraße 2, 30167 Hannover, Germany \\ Email: lechtenf@itp.uni-hannover.de, alexander.popov@itp.uni-hannover.de \\ ${ }^{2}$ Department of Mathematics, Heriot-Watt University \\ Colin Maclaurin Building, Riccarton, Edinburgh EH14 4AS, U.K. \\ and Maxwell Institute for Mathematical Sciences, Edinburgh, U.K. \\ and The Higgs Centre for Theoretical Physics, Edinburgh, U.K. \\ Email: R.J.Szabo@hw.ac.uk
}

\begin{abstract}
We consider SU(2)-equivariant dimensional reduction of Yang-Mills theory on manifolds of the form $M \times S^{3} / \Gamma$, where $M$ is a smooth manifold and $S^{3} / \Gamma$ is a three-dimensional Sasaki-Einstein orbifold. We obtain new quiver gauge theories on $M$ whose quiver bundles are based on the affine ADE Dynkin diagram associated to $\Gamma$. We relate them to those arising through translationallyinvariant dimensional reduction over the associated Calabi-Yau cones $C\left(S^{3} / \Gamma\right)$ which are based on McKay quivers and ADHM matrix models, and to those arising through $\mathrm{SU}(2)$-equivariant dimensional reduction over the leaf spaces of the characteristic foliations of $S^{3} / \Gamma$ which are Kähler orbifolds of $\mathbb{C} P^{1}$ whose quiver bundles are based on the unextended Dynkin diagram corresponding to $\Gamma$. We use Nahm equations to describe the vacua of $\mathrm{SU}(2)$-equivariant quiver gauge theories on the cones as moduli spaces of spherically symmetric instantons. We relate them to the Nakajima quiver varieties which can be realized as Higgs branches of the worldvolume quiver gauge theories on $\mathrm{D} p$-branes probing $\mathrm{D}(p+4)$-branes which wrap an ALE space, and to the moduli spaces of spherically symmetric solutions in putative non-abelian generalizations of two-dimensional affine Toda field theories.
\end{abstract}




\section{Contents}

1 Introduction and summary 1

2 Geometry of Sasaki-Einstein three-manifolds 3

3 Homogeneous vector bundles and quiver representations $\quad 11$

4 Equivariant dimensional reduction and quiver bundles $\quad 16$

5 Instantons on orbifolds and quiver varieties $\quad 20$

6 Instantons on cones and Nahm equations 26

\section{Introduction and summary}

Quiver gauge theories naturally arise through equivariant dimensional reduction over Kähler coset spaces $[1,2,3]$ where they find applications to Yang-Mills-Higgs theories and the construction of (non-abelian) vortices, and also as the low-energy effective field theories of D-branes at orbifold singularities $[4,5,6,7,8]$ which have important ramifications for the AdS/CFT correspondence and whose Higgs branches provide examples of singularity resolution in string geometry. In this paper we clarify the relations between these two seemingly disparate appearences of quiver gauge theories in a particular class of examples.

We consider the simplest coset $\mathbb{C} P^{1} \cong \mathrm{SU}(2) / \mathrm{U}(1)$ (and certain orbifolds thereof), whose associated quiver gauge theories are described in detail in [9] (see also [10, 11]); the underlying graph of the quiver bundles in this case is a Dynkin diagram of type $A_{k+1}$. To any Kähler manifold $Z$ one can associate a U(1)-bundle over $Z$ whose total space is a Sasaki-Einstein manifold with Calabi-Yau metric cone. In particular, when $Z=\mathbb{C} P^{1}$ any such Sasaki-Einstein manifold is isomorphic to an orbifold $S^{3} / \Gamma$ of the three-sphere for a finite subgroup $\Gamma$ of $\mathrm{SU}(2)$; when $\Gamma=\mathbb{Z}_{k+1}$ is a cyclic group then $S^{3} / \mathbb{Z}_{k+1}$ is a lens space. In this paper we describe new quiver gauge theories which can be associated with the Sasaki-Einstein manifolds $S^{3} / \Gamma$ via $\mathrm{SU}(2)$-equivariant dimensional reduction, which in certain limits reduce to those of [9]; the new quiver bundles are associated to quivers with vertex loop edges. We shall also describe quiver gauge theories associated to Calabi-Yau cones $C\left(S^{3} / \Gamma\right)$ over these spaces, which correspond to ADE orbifolds of $\mathbb{R}^{4}$. In translationally-invariant dimensional reduction such field theories are central in the description of D-branes at an orbifold singularity $\mathbb{R}^{4} / \Gamma$, and to the McKay correspondence for Calabi-Yau twofolds. The Sasaki-Einstein manifold $S^{3} / \Gamma$ interpolates between the two distinct Kähler manifolds $C\left(S^{3} / \Gamma\right)$ and $\mathbb{C} P^{1}$, and in this paper we use this bridge to clarify the dynamical relations between the McKay quiver bundles underlying these worldvolume field theories and the quiver bundles associated with the Kähler coset space $\mathbb{C} P^{1}$.

For this, we shall study the relations between vacua of these quiver gauge theories and instantons on the Calabi-Yau cones $C\left(S^{3} / \Gamma\right)$, which in translationally-invariant dimensional reduction in string theory correspond to D-branes located at points of the Calabi-Yau spaces $M_{\xi}$ which are minimal resolutions of the orbifold singularities $M_{0} \cong \mathbb{R}^{4} / \Gamma$ (where $\xi$ are Fayet-Iliopoulos terms serving as stability parameters of the resolution). In particular, the moduli spaces of translationally-invariant solutions of the Hermitian Yang-Mills equations on $\mathbb{R}^{4} / \Gamma$ coincide with the resolutions $M_{\xi}[12$, 13], which is described by a particular matrix model that can be promoted to an ADHM matrix model [14]. This correspondence forms the basis for the four-dimensional $\mathcal{N}=2$ superconformal 
quiver gauge theories on D-branes in which $M_{\xi}$ parameterize the supersymmetric vacuum states [4, $5,7]$; under the AdS/CFT correspondence, this gauge theory is dual to type IIB supergravity on an $A d S_{5} \times S^{5} / \Gamma$ background. More generally, in type II string theory the moduli space of instantons on the ALE space $M_{\xi}$ can be identified with the Higgs branch of the quiver gauge theory with eight real supercharges living on the worldvolume of $\mathrm{D} p$-branes probing a set of $\mathrm{D}(p+4)$-branes which wrap $M_{\xi}$.

On the other hand, the quiver gauge theories associated with the Calabi-Yau spaces $C\left(S^{3} / \Gamma\right)$ are technically much more involved, because instead of translational-invariance along $C\left(S^{3} / \Gamma\right)$ one imposes $\mathrm{SU}(2)$-equivariance along codimension one subspaces $S^{3} / \Gamma$ of the cones; the cones are $\mathrm{SU}(2)$-manifolds with one-dimensional orbit space parameterized by $r \in \mathbb{R}_{\geq 0}$ such that there is one singular orbit $S^{3} / \Gamma$ over $r=0$ and non-singular orbits $S^{3} / \Gamma$ for all $r>0$. The condition of $\mathrm{SU}(2)$-equivariance pulls the model back to the quiver gauge theories associated with $S^{3} / \Gamma$, but allows for a residual dependence on the radial coordinate $r$ which leads to Nahm-type equations for spherically symmetric instantons; it is important to note that these Nahm equations describe SU(2)invariant instantons on $C\left(S^{3} / \Gamma\right)$ and are not related with monopoles, a feature which somewhat obscures a direct realization in D-brane field theory. Thus instead of the ADHM-type matrix equations describing translationally-invariant vacua, imposing SU(2)-equivariance on gauge fields reduces the anti-self-duality equations on the four-dimensional cone to Nahm-type equations. This difference translates into significant differences between the well-known McKay quivers and our new "Sasakian" quivers which characterize equivariant dimensional reduction over the Sasaki-Einstein orbifolds.

Furthermore, the same moduli space $M_{\xi}$ appears as the moduli space of SU(2)-invariant instantons on the Calabi-Yau cone $\mathbb{R}^{4} \backslash\{0\}=C\left(S^{3}\right)$, in which case the anti-self-dual Yang-Mills equations reduce to the Nahm equations with suitable boundary conditions $[15,16,17]$. This is the moduli space of the spherically symmetric instanton which has the minimal fractional topological charge; these moduli spaces are further studied and extended to the cones $C\left(S^{3} / \Gamma\right)$ in [18]. Moduli spaces of solutions to Nahm equations with these boundary conditions also appear as Higgs moduli spaces of supersymmetric vacua in $\mathcal{N}=4$ supersymmetric Yang-Mills theory on the half-space $\mathbb{R}^{1,2} \times \mathbb{R}_{>0}$ with generalized Dirichlet boundary conditions [19]; these boundary conditions are realized by brane configurations in which $\mathrm{D} p$-branes end on $\mathrm{D}(p+2)$-branes at the boundary of $\mathbb{R}_{\geq 0}$. Thus the solutions of both the ADHM-type and Nahm-type equations give rise to the same moduli space $C\left(S^{3} / \Gamma\right)$, which after minimal resolution of singularities is the Calabi-Yau twofold $M_{\xi}$.

We shall show that by considering $\Gamma$-equivariant solutions to the Nahm equations, the moduli space can be described as a quiver variety via an infinite-dimensional hyper-Kähler quotient construction based on a flat hyper-Kähler Banach space factored by the action of an infinitedimensional group of gauge transformations; the resulting finite-dimensional quiver varieties are based on our Sasakian quivers and have orbifold singularities. On the other hand the ADHM construction describes a minimal resolution of the same moduli space, via a finite-dimensional hyper-Kähler quotient construction using constant matrices, as a quiver variety based on McKay quivers instead of our Sasakian quivers; the different occurences of quivers corresponds to the different constraints imposed on the matrices in the respective cases. In particular, we argue that the minimal charge instanton on $\mathbb{R}^{4} / \Gamma$ (or on its Calabi-Yau resolution) can be constructed both via the ADHM construction (reduced to the Kronheimer matrix equations) and by reduction to Nahm equations with respect to a radial coordinate on $\mathbb{R}^{4} / \Gamma$ (consistently with the construction on $\mathbb{R}^{4}$ ), wherein one obtains the same four-dimensional hyper-Kähler moduli space of vacua.

The outline of the remainder of this paper is as follows. In Sect. 2 we give a fairly self-contained account of the geometry of the Sasaki-Einstein orbifolds $S^{3} / \Gamma$ in a form that we will use in this paper. In Sect. 3 we derive the correspondence between homogeneous vector bundles on $S^{3} / \Gamma$ and representations of certain new quivers, which we call "Sasakian quivers" and which play a prominent 
role throughout the paper. In Sect. 4 we consider $\mathrm{SU}(2)$-equivariant dimensional reduction over the orbifold $S^{3} / \Gamma$, and derive the correspondence between $\mathrm{SU}(2)$-equivariant vector bundles on product manifolds $M \times S^{3} / \Gamma$ and quiver bundles ( $\Gamma$-equivariant vector bundles) on $M$ associated to the Sasakian quivers; the moduli spaces of vacua of the quiver gauge theories arising from reduction of Yang-Mills theory are described and it is shown how they reduce to the quiver gauge theories obtained via $\mathrm{SU}(2)$-equivariant dimensional reduction over $\mathbb{C} P^{1}$ (and certain orbifolds thereof). In Sect. 5 we describe the moduli spaces of translationally-invariant instantons on $\mathbb{R}^{4} / \Gamma$ in terms of Nakajima quiver varieties, and their connection to moduli spaces of instantons on an ALE space $M_{\xi}$ which are based on McKay quivers. Finally, in Sect. 6 we consider SU(2)-equivariant dimensional reduction over the cones $C\left(S^{3} / \Gamma\right)$ and study the Higgs branch of vacua of the induced quiver gauge theories as moduli spaces of solutions to Nahm equations which are based on extensions of quiver varieties to the setting of Sasakian quivers; we discuss their relations to the ADHM-type moduli spaces and to certain Nakajima quiver varieties of $A_{n}$-type, and also their realization in a variant of non-abelian affine Toda field theory which extends the duality between four-dimensional gauge theories and two-dimensional conformal field theories.

\section{Geometry of Sasaki-Einstein three-manifolds}

Sphere $S^{3}$. Let $S^{3}$ be the standard round three-sphere of radius $r$. It can be described via the embedding $S^{3} \subset \mathbb{R}^{4}$ by the equation

$$
\delta_{\mu \nu} y^{\mu} y^{\nu}=r^{2},
$$

where $y^{\mu} \in \mathbb{R}$ and $\mu, \nu, \ldots=1,2,3,4$. On $S^{3}$ one can introduce a basis of left $\mathrm{SU}(2)$-invariant one-forms $\left\{e^{a}\right\}$ as

$$
e^{a}:=-\frac{1}{r^{2}} \bar{\eta}_{\mu \nu}^{a} y^{\mu} \mathrm{d} y^{\nu}
$$

where $\bar{\eta}_{\mu \nu}^{a}$ are the anti-self-dual 't Hooft tensors

$$
\bar{\eta}_{b c}^{a}=\varepsilon_{b c}^{a} \quad \text { and } \quad \bar{\eta}_{b 4}^{a}=-\bar{\eta}_{4 b}^{a}=-\delta_{b}^{a},
$$

with $\varepsilon_{23}^{1}=1$ and $a, b, \ldots=1,2,3$. These one-forms satisfy the Maurer-Cartan equations

$$
\mathrm{d} e^{a}+\varepsilon_{b c}^{a} e^{b} \wedge e^{c}=0,
$$

and the round metric on $S^{3}$ is given by

$$
\mathrm{d} s_{S^{3}}^{2}=r^{2} \delta_{a b} e^{a} \otimes e^{b} .
$$

Sasaki-Einstein orbifolds $\boldsymbol{S}^{\mathbf{3}} / \boldsymbol{\Gamma}$. Sasakian manifolds are the natural odd-dimensional counterparts of Kähler manifolds; a Riemannian manifold is Sasakian if its associated metric cone is Kähler. A Sasakian manifold is Sasaki-Einstein if its Riemannian metric is an Einstein metric, or equivalently if its metric cone is a Calabi-Yau space. Thus Sasaki-Einstein spaces are the natural odd-dimensional versions of Kähler-Einstein spaces. For background on Sasaki-Einstein manifolds, see e.g. [20].

Three-dimensional Sasaki-Einstein spaces are completely classified. They correspond to the homogeneous spherical space forms in three dimensions, which are the smooth orbifolds $S^{3} / \Gamma$ where $\Gamma$ is a finite subgroup of $\mathrm{SU}(2)$ commuting with $\mathrm{U}(1) \subset \mathrm{SU}(2)$ which acts freely and isometrically by left translations on the group manifold $\mathrm{SU}(2) \cong S^{3}$; by the McKay correspondence they have an ADE classification and we run through the complete list below. The orbifold $S^{3} / \Gamma$ has a description as a Seifert fibration, i.e. as a U(1) V-bundle

$$
\pi_{\Gamma}: S^{3} / \Gamma \longrightarrow \mathbb{C} P^{1} / \Gamma_{0},
$$


which is called the characteristic foliation of the Sasaki-Einstein space. The space of leaves $\mathbb{C} P^{1} / \Gamma_{0}$ is a one-dimensional complex orbifold with a Kähler-Einstein metric; the ADE group $\Gamma$ is a central extension (by rotations of the regular fibres) of the group $\Gamma_{0} \subset \mathrm{SO}(3)$ which acts isometrically on a local coordinate $z \in \mathbb{C}$ of the Riemann sphere $\mathbb{C} P^{1} \cong S^{2}$ by $\mathrm{SU}(2)$ Möbius transformations. Concretely, the orbifold base $\mathbb{C} P^{1} / \Gamma_{0}$ can be regarded as a Riemann sphere together with $m$ arbitrary marked points whose coordinate charts are modelled on $\mathbb{C} / \mathbb{Z}_{k_{j}}$ for some ramification indices $k_{j} \geq 0$ with $j=1, \ldots, m$. Let $\mathcal{L}_{\Gamma} \rightarrow \mathbb{C} P^{1} / \Gamma_{0}$ be the line $\mathrm{V}$-bundle associated to the Seifert fibration (2.6); it is defined by the identifications

$$
(z, w) \sim\left(\zeta_{k_{j}} z, \zeta_{k_{j}}^{l_{j}} w\right) \quad \text { with } \quad \zeta_{k_{j}}=\exp \left(2 \pi \mathrm{i} / k_{j}\right)
$$

of the local coordinates $(z, w) \in \mathbb{C}^{2}$ of the base and fibre around the $j$-th orbifold point, for some integer isotropy weights $0 \leq l_{j}<k_{j}$. Note that $\operatorname{deg}\left(\mathcal{L}_{\Gamma}\right)=c_{1}\left(\left|\mathcal{L}_{\Gamma}\right|\right)=d \in \mathbb{Z}$, where $\left|\mathcal{L}_{\Gamma}\right| \rightarrow \mathbb{C} P^{1}$ is the associated smooth line bundle obtained by smoothing the orbifold points and eliminating the monodromies $l_{j}=0$; on the other hand, the orbifold Chern class of $\mathcal{L}_{\Gamma}$ is given by

$$
c_{1}\left(\mathcal{L}_{\Gamma}\right)=d+\sum_{j=1}^{m} \frac{l_{j}}{k_{j}} \in \mathbb{Q}_{>0} .
$$

The locally free $\mathrm{U}(1)$-action on $S^{3} / \Gamma$ arises from rotations in the fibres over $\mathbb{C} P^{1} / \Gamma_{0}$, with the fibre over the $j$-th ramification point of $\mathbb{C} P^{1} / \Gamma_{0}$ given by $S^{1} / \mathbb{Z}_{k_{j}}$ due to the orbifold identification (2.7). Since $S^{3}$ is simply connected and $\Gamma$ acts freely, the fundamental group of $S^{3} / \Gamma$ is the finite group $\Gamma$ itself. Using the standard presentation of $\pi_{1}\left(S^{3} / \Gamma\right)$ in terms of generators and relations associated to the Seifert invariants, this gives a geometric presentation of the orbifold group $\Gamma$ in terms of the central generator $h$ along the generic $S^{1}$ fibre over $\mathbb{C} P^{1} / \Gamma_{0}$ together with one-cycles $\xi_{j}$ for $j=1, \ldots, m$ encircling the orbifold points on $\mathbb{C} P^{1} / \Gamma_{0}$, with the relations

$$
\xi_{j}^{k_{j}}=h^{-l_{j}} \quad \text { and } \quad \xi_{1} \cdots \xi_{m}=h^{d} .
$$

In particular, by setting $h=1$ this yields a presentation of the orbifold fundamental group $\Gamma_{0}=$ $\pi_{1}\left(\mathbb{C} P^{1} / \Gamma_{0}\right)$ in terms of generators $\xi_{j}$ with relations.

By working on the uniformizing system of local charts of the orbifold $\mathbb{C} P^{1} / \Gamma_{0}$, we can define a local basis of one-forms for $S^{3} / \Gamma$ as follows. For this, we use the Seifert description of $S^{3}$ as the total space of the circle bundle of the line bundle $\mathcal{L} \rightarrow \mathbb{C} P^{1}$ of degree one corresponding to the Hopf fibration $S^{3} \rightarrow S^{2}$. Consider the $(k+1)$-tensor power $\mathcal{L}^{k+1}:=(\mathcal{L})^{\otimes(k+1)}$ of $\mathcal{L}$, which is the Hermitian line bundle

$$
\mathcal{L}^{k+1} \longrightarrow \mathbb{C} P^{1}
$$

with the unique $\mathrm{SU}(2)$-invariant connection $a_{k+1}$ having in local coordinates the form

$$
a_{k+1}=(k+1) a_{1}=\frac{k+1}{2(1+z \bar{z})}(\bar{z} \mathrm{~d} z-z \mathrm{~d} \bar{z}) .
$$

Let

$$
\beta=\frac{\mathrm{d} z}{1+z \bar{z}} \quad \text { and } \quad \bar{\beta}=\frac{\mathrm{d} \bar{z}}{1+z \bar{z}}
$$

be the unique $\mathrm{SU}(2)$-invariant forms of types $(1,0)$ and $(0,1)$ on $\mathbb{C} P^{1}$; they form a basis of covariantly constant sections of the canonical line bundles $K=\mathcal{L}^{2}$ and $K^{-1}=\mathcal{L}^{-2}$ obeying

$$
\mathrm{d} \beta-a_{2} \wedge \beta=0 \quad \text { and } \quad \mathrm{d} \bar{\beta}-a_{-2} \wedge \bar{\beta}=0 .
$$


The SU(2)-invariant Kähler (1,1)-form on $\mathbb{C} P^{1}$ is $\omega=\frac{\mathrm{i}}{2} r^{2} \beta \wedge \bar{\beta}$. Then basis one-forms on $S^{3} / \Gamma$ can be introduced via

$$
e^{1}+\mathrm{i} e^{2}=\pi_{\Gamma}^{*} \beta \quad \text { and } \quad e^{3}=\frac{1}{d}\left(\mathrm{~d} \varphi-\mathrm{i} c_{1}\left(\mathcal{L}_{\Gamma}\right) \pi_{\Gamma}^{*} a_{1}\right),
$$

where $0 \leq \varphi<2 \pi$ is a local coordinate on the $S^{1}$ fibre of the Seifert bundle (2.6). The frame element $e^{3}$ is a connection one-form on the Seifert fibration with curvature

$$
\mathrm{d} e^{3}=\frac{2}{d r^{2}} c_{1}\left(\mathcal{L}_{\Gamma}\right) \pi_{\Gamma}^{*} \omega
$$

From (2.13) and (2.15) we obtain similar Maurer-Cartan equations (2.4) for $e^{a}$ as on $S^{3}$. In particular, the Sasaki-Einstein metric on $S^{3} / \Gamma$ is given by

$$
\mathrm{d} s_{S^{3} / \Gamma}^{2}=\pi_{\Gamma}^{*} \mathrm{~d} s_{\mathbb{C} P^{1}}^{2}+r^{2} e^{3} \otimes e^{3},
$$

where $\mathrm{d} s_{\mathbb{C} P^{1}}^{2}=r^{2} \beta \otimes \bar{\beta}$ is the Kähler-Einstein metric on $\mathbb{C} P^{1}$.

Calabi-Yau cones $C\left(S^{3} / \Gamma\right)$. Our Sasaki-Einstein orbifolds can be defined as the bases of affine Calabi-Yau cones described by polynomial equations in $\mathbb{C}^{3}$. The equation (2.1) defines the embedding of $S^{3}$ into the space $\mathbb{R}^{4} \cong \mathbb{C}^{2}$ with the complex coordinates

$$
z^{1}=y^{1}+\mathrm{i} y^{2} \quad \text { and } \quad z^{2}=y^{3}+\mathrm{i} y^{4} .
$$

In fact, $\mathbb{R}^{4}$ can be considered as a cone $C\left(S^{3}\right)$ over $S^{3}$,

$$
\mathbb{R}^{4} \backslash\{0\}=C\left(S^{3}\right)
$$

with the metric

$$
\mathrm{d} s_{C\left(S^{3}\right)}^{2}=\mathrm{d} r^{2}+r^{2} \delta_{a b} e^{a} \otimes e^{b}
$$

where $r^{2}=\delta_{\mu \nu} y^{\mu} y^{\nu}$ and $e^{a}$ are the one-forms (2.2) on $S^{3}$. We can identify $\mathbb{R}^{4} \cong \mathbb{C}^{2}$ with the affine subvariety cut out by the linear relation $f(x, y, z)=x+y+z=0$ in $\mathbb{C}^{3}$; by rescaling the affine coordinates $(x, y, z) \in \mathbb{C}^{3}$ by $\lambda \in \mathbb{C}^{*}$, the polynomial $f(x, y, z)$ may also be regarded as cutting out a copy of the Riemann sphere $S^{2} \cong \mathbb{C} P^{1} \subset \mathbb{C} P^{2}$, and in this way the Calabi-Yau cone describes the standard Hopf fibration $S^{3} \rightarrow S^{2}$. In this case the spheres $S^{3}$ of varying radii are the natural invariant submanifolds for the action of the Lorentz group $\mathrm{SO}(4) \cong \mathrm{SU}(2) \times \mathrm{SU}(2)$ on $\mathbb{R}^{4}$, in the sense that the orbits of the free left action of $\mathrm{SU}(2)$ on $\left(z^{1}, z^{2}\right)$ parameterize families of three-spheres via the cone relation (2.18).

Consider the action of $\Gamma$ on $\mathbb{C}^{2}$ given by

$$
\left(z^{1}, z^{2}\right) \longmapsto\left(g_{\alpha}^{1} z^{\alpha}, g_{\alpha}^{2} z^{\alpha}\right)
$$

for $\alpha, \beta, \ldots=1,2$, where $g^{\alpha}{ }_{\beta}$ are the matrix elements of $g \in \Gamma$ in the fundamental two-dimensional representation of $\Gamma \subset \mathrm{SU}(2)$ on $\mathbb{C}^{2}$. It has a single isolated fixed point at the origin $\left(z^{1}, z^{2}\right)=(0,0)$. The orbifold $\mathbb{C}^{2} / \Gamma$ is defined as the set of equivalence classes with respect to the equivalence relation

$$
\left(g^{1}{ }_{\alpha} z^{\alpha}, g^{2}{ }_{\alpha} z^{\alpha}\right) \equiv\left(z^{1}, z^{2}\right)
$$

for all $g \in \Gamma$. It has a singularity at the origin and is a cone over $S^{3} / \Gamma$,

$$
\left(\mathbb{C}^{2} \backslash\{0\}\right) / \Gamma=C\left(S^{3} / \Gamma\right),
$$

with the metric (2.19) for $e^{a}$ given in (2.14). 
A natural description of the cone (2.22) is as the complex surface in $\mathbb{C}^{3}$ which is invariant under the $\mathbb{C}^{*}$-action given by $(x, y, z) \mapsto\left(\lambda^{w_{x}} x, \lambda^{w_{y}} y, \lambda^{w_{z}} z\right)$; due to the scaling symmetry with respect to $\lambda \in \mathbb{C}^{*}=\mathbb{R}_{>0} \times S^{1}$ these spaces are cones, and because of the $\mathbb{R}_{>0}$-action the Calabi-Yau metric can be written in the form (2.19). Explicitly, it can be defined as the subvariety cut out by a polynomial equation

$$
f_{\Gamma}(x, y, z)=0
$$

where $f_{\Gamma}(x, y, z)$ is a weighted homogeneous polynomial, i.e. $f_{\Gamma}\left(\lambda^{w_{x}} x, \lambda^{w_{y}} y, \lambda^{w_{z}} z\right)=\lambda^{n} f_{\Gamma}(x, y, z)$ for all $\lambda \in \mathbb{C}^{*}$. The degree $n>0$ coincides with the dual Coxeter number of the corresponding ADE Lie group, while the pairwise coprime weights $w_{x}, w_{y}, w_{z} \in \mathbb{Z}_{\geq 0}$ are divisors of $n$ with $|\Gamma|=2 w_{x} w_{y}$ and $w_{z}=\frac{n}{2}=w_{x}+w_{y}-1$. These integers can be expressed in terms of representation theory data of the orbifold group $\Gamma$ : If $n_{\ell}$ for $\ell=0,1, \ldots, r_{\Gamma}$ is the dimension of the $\ell$-th irreducible representation $V_{\ell}$ of $\Gamma$, where $r_{\Gamma}$ is the rank of the corresponding ADE Lie group and $\ell=0$ labels the trivial representation with $n_{0}=1$, then

$$
n=\sum_{\ell=0}^{r_{\Gamma}} n_{\ell} \quad \text { and } \quad|\Gamma|=\sum_{\ell=0}^{r_{\Gamma}} n_{\ell}^{2} .
$$

The base of the cone is the intersection of $\mathbb{C}^{2} / \Gamma$ with the sphere $S^{5} \subset \mathbb{C}^{3}$ of radius $r$, which is just $S^{3} / \Gamma$. The characteristic foliation (2.6) is then generated by the remaining U(1)-action on $S^{3} / \Gamma$ inherited from the original $\mathbb{C}^{*}$-action on the cone. In fact, this construction provides an alternative realization of the leaf space $\mathbb{C} P^{1} / \Gamma_{0}$ as a quasi-smooth weighted projective curve cut out by the same polynomial equation $(2.23)$, but now regarded in the weighted projective plane $\mathbb{C} P^{2}\left(w_{x}, w_{y}, w_{z}\right)$. This yields a natural description of $\mathbb{C} P^{1} / \Gamma_{0}=\left(S^{3} / \Gamma\right) / S^{1}$ as the complex orbifold

$$
\mathbb{C} P^{1} / \Gamma_{0}=C\left(S^{3} / \Gamma\right) / \mathbb{C}^{*}=\left(\left(\mathbb{C}^{2} \backslash\{0\}\right) / \Gamma\right) / \mathbb{C}^{*} .
$$

$\Gamma$-action on one-forms. Consider a one-form

$$
Y=Y_{\mu} \mathrm{d} y^{\mu}=Y_{z^{1}} \mathrm{~d} z^{1}+Y_{z^{2}} \mathrm{~d} z^{2}+Y_{\bar{z}^{\overline{1}}} \mathrm{~d} \bar{z}^{\overline{1}}+Y_{\bar{z}^{2}} \mathrm{~d} \bar{z}^{\overline{2}}
$$

on $\mathbb{R}^{4} \cong \mathbb{C}^{2}$ which is invariant under the action of $\Gamma \subset \mathrm{SU}(2) \subset \mathrm{SO}(4)$ defined by (2.20). Then for the components

$$
Y_{z^{1}}=\frac{1}{2}\left(Y_{1}-\mathrm{i} Y_{2}\right) \quad \text { and } \quad Y_{z^{2}}=\frac{1}{2}\left(Y_{3}-\mathrm{i} Y_{4}\right)
$$

we have

$$
Y_{z^{1}} \longmapsto\left(g^{-1}\right)_{1}^{\alpha} Y_{z^{\alpha}} \quad \text { and } \quad Y_{z^{2}} \longmapsto\left(g^{-1}\right)_{2}{ }^{\alpha} Y_{z^{\alpha}}
$$

for all $g \in \Gamma$. On the other hand, for the components $\left(X_{a}, X_{r}\right)$ in spherical coordinates, defined as

$$
\begin{aligned}
Y=Y_{\mu} \mathrm{d} y^{\mu}=: & X_{a} e^{a}+X_{r} \mathrm{~d} r \\
& =\frac{1}{2}\left(X_{1}-\mathrm{i} X_{2}\right)\left(e^{1}+\mathrm{i} e^{2}\right)+\frac{1}{2}\left(X_{3}-\mathrm{i} r X_{r}\right)\left(e^{3}+\mathrm{i} \frac{\mathrm{d} r}{r}\right)+\text { h.c. },
\end{aligned}
$$

the $\Gamma$-action on $Y_{\mu}$ implies

$$
X_{1}+\mathrm{i} X_{2} \longmapsto \pi(g)\left(X_{1}+\mathrm{i} X_{2}\right) \quad \text { and } \quad X_{3}+\mathrm{i} r X_{r} \longmapsto \pi(g)\left(X_{3}\right)+\mathrm{i} r X_{r},
$$

which defines the representation $\pi: \Gamma \rightarrow \operatorname{End}_{C^{\infty}\left(S^{3}\right)}\left(\Omega_{S^{3}}^{1}\right)$ of the orbifold group on one-forms on $S^{3}$. Once the transformations (2.20) are given explicitly, the transformations (2.30) can be worked out from the formulas (2.2) which in the coordinates (2.17) have the form

$$
e^{1}+\mathrm{i} e^{2}=\frac{\mathrm{i}}{r^{2}}\left(z^{1} \mathrm{~d} \bar{z}^{\overline{2}}-\bar{z}^{\overline{2}} \mathrm{~d} z^{1}\right) \quad \text { and } \quad e^{3}=\frac{\mathrm{i}}{2 r^{2}}\left(\bar{z}^{\overline{1}} \mathrm{~d} z^{1}+z^{2} \mathrm{~d} \bar{z}^{\overline{2}}-z^{1} \mathrm{~d} \bar{z}^{\overline{1}}-\bar{z}^{\overline{2}} \mathrm{~d} z^{2}\right) .
$$


Note that $X_{a}$ and $X_{\tau}:=r X_{r}$ for $\tau=\log r$ can be considered as components of one-forms on the cylinder $\mathbb{R} \times S^{3} / \Gamma$ with the metric

$$
\mathrm{d} s_{\mathbb{R} \times S^{3} / \Gamma}^{2}=\mathrm{d} \tau^{2}+\delta_{a b} e^{a} \otimes e^{b}=\frac{\mathrm{d} r^{2}}{r^{2}}+\delta_{a b} e^{a} \otimes e^{b}=\frac{1}{r^{2}} \mathrm{~d} s_{C\left(S^{3} / \Gamma\right)}^{2}
$$

where $\mathrm{d} r=\mathrm{d}\left(z^{1} \bar{z}^{\overline{1}}+z^{2} \bar{z}^{2}\right)^{1 / 2}$, which is conformally equivalent to the metric (2.19) on the cone (2.22) for $e^{a}$ given in (2.14).

$\boldsymbol{A}_{\boldsymbol{k}}$. In this case $\Gamma=\mathbb{Z}_{k+1}$ for $k \geq 1$ is the cyclic group of order $k+1$, generated by an element $h$ with $h^{k+1}=1$, and the quotient space $S^{3} / \mathbb{Z}_{k+1}$ is the lens space $L(k+1,1)$; in this instance $\Gamma_{0}$ is the trivial group. It can be identified with the total space of the $\mathrm{U}(1)$-bundle associated to the line bundle (2.10), regarded as an $S^{1} / \mathbb{Z}_{k+1}$-fibration over $\mathbb{C} P^{1}$. Basis one-forms on this space can be constructed explicitly by using a local section of the circle bundle of the bundle (2.10) given by the matrix

$$
g(z, \bar{z})=\frac{1}{(1+z \bar{z})^{1 / 2}}\left(\begin{array}{cc}
1 & -\bar{z} \\
z & 1
\end{array}\right)\left(\begin{array}{cc}
\exp \left(\frac{\mathrm{i} \varphi}{k+1}\right) & 0 \\
0 & \exp \left(-\frac{\mathrm{i} \varphi}{k+1}\right)
\end{array}\right) \in \mathrm{SU}(2)
$$

where $0 \leq \varphi<2 \pi$ is a local coordinate on $S^{1}$ and $\exp \left(\frac{i \varphi}{k+1}\right) \in S^{1} / \mathbb{Z}_{k+1}$. On the trivial rank two complex vector bundle $S^{3} / \mathbb{Z}_{k+1} \times \mathbb{C}^{2}$ over $S^{3} / \mathbb{Z}_{k+1}$ we introduce the flat connection

$$
B:=g^{-1} \mathrm{~d} g=\left(\begin{array}{cc}
\frac{\mathrm{i}}{k+1} \mathrm{~d} \varphi+a_{1} & -\exp \left(-\frac{2 \mathrm{i} \varphi}{k+1}\right) \bar{\beta} \\
\exp \left(\frac{2 \mathrm{i} \varphi}{k+1}\right) \beta & -\frac{\mathrm{i}}{k+1} \mathrm{~d} \varphi-a_{1}
\end{array}\right)=:\left(\begin{array}{cc}
\mathrm{i} e^{3} & -e^{1}+\mathrm{i} e^{2} \\
e^{1}+\mathrm{i} e^{2} & -\mathrm{i} e^{3}
\end{array}\right),
$$

which is an $\mathfrak{s u}(2)$-valued one-form on $S^{3} / \mathbb{Z}_{k+1}$. From flatness of the connection (2.34), $\mathrm{d} B+B \wedge B=$ 0 , we obtain the same Maurer-Cartan equations (2.4) for $e^{a}$ as on $S^{3}$, i.e. for $k=0$.

The relevant group theory data is encoded in the extended simply laced Dynkin diagram $A_{k}$ of the affine $\widehat{A}_{k}$ Lie algebra given by

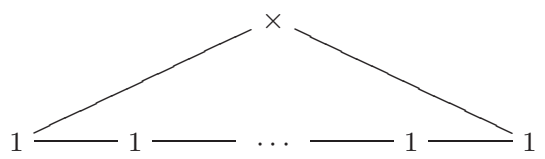

with $r_{\Gamma}+1=k+1$ nodes designating the irreducible representations of $\Gamma$; here and in the following the integers at the nodes are the Dynkin indices $n_{\ell}$ of the affine roots, and the node marked $\times$ corresponds to the trivial representation with $n_{0}=1$. Any unitary representation of $\mathbb{Z}_{k+1}$ is given by a sum of one-dimensional irreducible representations $V_{\ell}$ with $\ell=0,1, \ldots, k(\bmod (k+1))$ on which the generator $h$ acts as multiplication by

$$
\zeta_{k+1}^{\ell}:=\exp \left(\frac{2 \pi \mathrm{i} \ell}{k+1}\right)
$$

As a subgroup of $\mathrm{SU}(2)$, the generating element of $\mathbb{Z}_{k+1}$ is given by

$$
\left(h^{\alpha}{ }_{\beta}\right)=\left(\begin{array}{cc}
\zeta_{k+1} & 0 \\
0 & \zeta_{k+1}^{-1}
\end{array}\right)
$$

and the transformation (2.30) in this case reads as

$$
\pi(h)\left(X_{1}+\mathrm{i} X_{2}\right)=\zeta_{k+1}^{2}\left(X_{1}+\mathrm{i} X_{2}\right) \quad \text { and } \quad \pi(h)\left(X_{3}\right)=X_{3} .
$$


Alternatively, the transformation (2.38) can be derived immediately from the definition (2.34) by considering the fibre monodromy $\varphi \mapsto \varphi+2 \pi$ which generates

$$
e^{1} \pm \mathrm{i} e^{2} \longmapsto \zeta_{k+1}^{ \pm 2}\left(e^{1} \pm \mathrm{i} e^{2}\right) \quad \text { and } \quad e^{3} \longmapsto e^{3} .
$$

The pertinent weighted homogeneous polynomial is

$$
f_{\mathbb{Z}_{k+1}}(x, y, z)=x^{k+1}+y^{2}+z^{2},
$$

with the weights

$$
w_{x}=1 \quad \text { and } \quad w_{y}=w_{z}=q+1 \quad \text { for } \quad k=2 q+1
$$

and

$$
w_{x}=2 \quad \text { and } \quad w_{y}=w_{z}=2 q+1 \quad \text { for } \quad k=2 q .
$$

Note that in the special case $k=1$ where $S^{3} / \mathbb{Z}_{2}=\mathrm{SU}(2) / \mathbb{Z}_{2}=\mathrm{SO}(3)=\mathbb{R} P^{3}$, this provides a realization of the complex projective line $\mathbb{C} P^{1}$ as the smooth Stenzel curve $x^{2}+y^{2}+z^{2}=0$ in $\mathbb{C} P^{2}$.

$\boldsymbol{D}_{\boldsymbol{k}}$. In this case $\Gamma=\mathbb{D}_{k-2}^{*}$ for $k \geq 4$ is the binary extension of the dihedral group $\Gamma_{0}=\mathbb{D}_{k-2}$ in $\mathrm{SU}(2)$ of order $4(k-2)$, and $S^{3} / \mathbb{D}_{k-2}^{*}$ is a prism manifold; the orbifold group $\Gamma$ in this case is the pullback of $\Gamma_{0}$ under the covering homomorphism $S^{3} \rightarrow \mathrm{SO}(3)=\mathrm{SU}(2) / \mathbb{Z}_{2}$ of degree two. It has a presentation as a non-abelian group generated by elements $\xi_{1}, \xi_{2}, \xi_{3}$ with the relations

$$
\xi_{1}^{2}=\xi_{2}^{2}=\xi_{3}^{k-2}=\xi_{1} \xi_{2} \xi_{3} .
$$

Note that $\xi_{3}$ generates a cyclic subgroup $\mathbb{Z}_{2(k-2)}$, and $\xi_{1}=\xi_{2} \xi_{3}$. The center of this group has order two and is generated by the element $h=\xi_{1} \xi_{2} \xi_{3}$; whence the generic fibre of the Seifert manifold $S^{3} / \mathbb{D}_{k-2}^{*}$ is $S^{1} / \mathbb{Z}_{2}$, and the geometry is analogous to that of the lens space $S^{3} / \mathbb{Z}_{2}$. The branching indices $\left(k_{1}, k_{2}, k_{3}\right)$ on $\mathbb{C} P^{1} / \mathbb{D}_{k-2}$ are given by the elliptic triple $(2,2, k-2)$, and so there are three exceptional fibres $S^{1} / \mathbb{Z}_{2}, S^{1} / \mathbb{Z}_{2}$, and $S^{1} / \mathbb{Z}_{k-2}$, respectively, each with isotropy one; in particular the base orbifold group is a $(2,2, k-2)$ triangle group. Moreover, the degree of the line V-bundle $\mathcal{L}_{\mathbb{D}_{k-2}^{*}} \rightarrow \mathbb{C} P^{1} / \mathbb{D}_{k-2}$ is $d=1$; whence the underlying smooth line bundle is $\left|\mathcal{L}_{\mathbb{D}_{k-2}^{*}}\right|=\mathcal{L}$ and the orbifold Chern class is given by

$$
c_{1}\left(\mathcal{L}_{\mathbb{D}_{k-2}^{*}}\right)=2+\frac{1}{k-2} .
$$

The extended simply laced Dynkin diagram $\mathrm{D}_{k}$ for the affine $\widehat{D}_{k}$ Lie algebra is

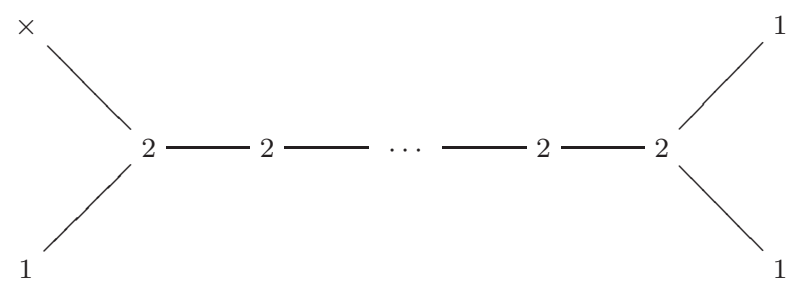

with $r_{\Gamma}+1=k+1$ nodes. As elements of $\mathrm{SU}(2)$ the generators of $\mathbb{D}_{k-2}^{*}$ are given by

$$
\left(\xi_{2}{ }^{\alpha} \beta\right)=\left(\begin{array}{cc}
0 & 1 \\
-1 & 0
\end{array}\right) \quad \text { and } \quad\left(\xi_{3}{ }^{\alpha} \beta\right)=\left(\begin{array}{cc}
\zeta_{2(k-2)} & 0 \\
0 & \zeta_{2(k-2)}^{-1}
\end{array}\right)
$$

together with $\xi_{1}=\xi_{2} \xi_{3}$; in this representation the central element is $h=-\mathbf{1}_{\mathbb{C}^{2}}$. The transformations (2.30) under the generator $\xi_{3}$ are analogous to those of (2.38), while under $\xi_{2}$ one has

$$
\pi\left(\xi_{2}\right)\left(X_{1}\right)=-X_{1}, \quad \pi\left(\xi_{2}\right)\left(X_{2}\right)=X_{2} \quad \text { and } \quad \pi\left(\xi_{2}\right)\left(X_{3}\right)=-X_{3} .
$$


The prism manifold $S^{3} / \mathbb{D}_{k-2}^{*}$ can also be described as the weighted projective curve

$$
f_{\mathbb{D}_{k-2}^{*}}(x, y, z)=x^{k-1}+x y^{2}+z^{2}=0 \quad \text { in } \quad \mathbb{C} P^{2}(2, k-2, k-1) .
$$

$\boldsymbol{E}_{6}$. In this case $\Gamma=\mathbb{T}^{*} \cong \mathrm{SL}\left(2, \mathbb{Z}_{3}\right)$ is the binary tetrahedral group of order 24 which is a central extension of the tetrahedral group $\Gamma_{0}=\mathbb{T} \cong A_{4}$ by a cyclic group $\mathbb{Z}_{2}$. It has a presentation in terms of generators $\xi_{1}, \xi_{2}, \xi_{3}$ with relations

$$
\xi_{1}^{2}=\xi_{2}^{3}=\xi_{3}^{3}=\xi_{1} \xi_{2} \xi_{3}
$$

Again the order two center of $\mathbb{T}^{*}$ is generated by $h=\xi_{1} \xi_{2} \xi_{3}$, and the branching indices on $\mathbb{C} P^{1} / \mathbb{T}$ are given by the Platonic triple $(2,3,3)$; whence $\Gamma_{0}=\mathbb{T}$ is a triangle group, and there are three exceptional fibres $S^{1} / \mathbb{Z}_{2}, S^{1} / \mathbb{Z}_{3}$, and $S^{1} / \mathbb{Z}_{3}$ with monodromy one. The line V-bundle $\mathcal{L}_{\mathbb{T}^{*}} \rightarrow$ $\mathbb{C} P^{1} / \mathbb{T}$ with $\left|\mathcal{L}_{\mathbb{T}^{*}}\right|=\mathcal{L}$ has orbifold Chern class

$$
c_{1}\left(\mathcal{L}_{\mathbb{T}^{*}}\right)=\frac{13}{6} .
$$

The extended simply laced Dynkin diagram $\mathrm{E}_{6}$ for the affine $\widehat{E}_{6}$ Lie algebra is

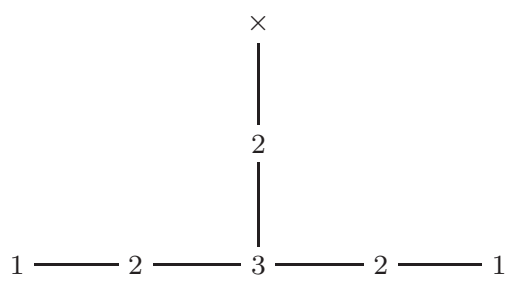

It is straightforward to write down elements of $\mathrm{SU}(2)$ for the generators $\xi_{j}$ of $\mathbb{T}^{*}$. However, for most practical calculations it is more convenient to note that the group $\mathbb{T}^{*} \subset \mathrm{SU}(2)$ is generated by the order eight dicyclic group $\mathbb{D}_{2}^{*}$ and the additional generator

$$
\left(g^{\alpha}{ }_{\beta}\right)=\frac{1}{\sqrt{2}}\left(\begin{array}{cc}
\zeta_{8}^{-1} & \zeta_{8}^{-1} \\
-\zeta_{8} & \zeta_{8}
\end{array}\right)=\frac{1}{1-\mathrm{i}}\left(\begin{array}{cc}
1 & 1 \\
-\mathrm{i} & \mathrm{i}
\end{array}\right)
$$

satisfying $g^{3}=-\mathbf{1}_{\mathbb{C}^{2}}$. The additional transformations by $g$ in $(2.30)$ read as

$$
\pi(g)\left(X_{1}\right)=X_{2}, \quad \pi(g)\left(X_{2}\right)=-X_{3} \quad \text { and } \quad \pi(g)\left(X_{3}\right)=-X_{1} .
$$

The Sasaki-Einstein manifold $S^{3} / \mathbb{T}^{*}$ also has a presentation as the weighted projective curve

$$
f_{\mathbb{T}^{*}}(x, y, z)=x^{4}+y^{3}+z^{2}=0 \quad \text { in } \quad \mathbb{C} P^{2}(3,4,6) .
$$

$\boldsymbol{E}_{\mathbf{7}}$. In this case $\Gamma=\mathbb{O}^{*}$ is the binary octahedral group of order 48 which is a central extension of the octahedral group $\Gamma_{0}=\mathbb{O} \cong S_{4}$ by a cyclic group $\mathbb{Z}_{2}$. It has a presentation in terms of generators $\xi_{1}, \xi_{2}, \xi_{3}$ with relations

$$
\xi_{1}^{2}=\xi_{2}^{3}=\xi_{3}^{4}=\xi_{1} \xi_{2} \xi_{3}
$$

The order two center of $\mathbb{O}^{*}$ is generated by $h=\xi_{1} \xi_{2} \xi_{3}$, and the branching indices on $\mathbb{C} P^{1} / \mathbb{O}$ are given by the Platonic triple $(2,3,4)$; whence there are three exceptional fibres $S^{1} / \mathbb{Z}_{2}, S^{1} / \mathbb{Z}_{3}$, and 
$S^{1} / \mathbb{Z}_{4}$ each of isotropy one. The line V-bundle $\mathcal{L}_{\mathbb{O}^{*}} \rightarrow \mathbb{C} P^{1} / \mathbb{O}$ with $\left|\mathcal{L}_{\mathbb{O}^{*}}\right|=\mathcal{L}$ has orbifold Chern class

$$
c_{1}\left(\mathcal{L}_{\mathbb{O}^{*}}\right)=\frac{25}{12} .
$$

The extended simply laced Dynkin diagram $E_{7}$ for the affine exceptional $\widehat{E}_{7}$ Lie algebra is

$$
\times-2-3-4-3-2-1
$$

The representation of $\mathbb{O}^{*}$ in $\mathrm{SU}(2)$ can be obtained similarly to that of $\mathbb{T}^{*}$ by extending the order 16 dicyclic group $\mathbb{D}_{4}^{*}$ by the same generator $(2.52)$. The spherical three-manifold $S^{3} / \mathbb{O}^{*}$ also has a presentation as the weighted projective curve

$$
f_{\mathbb{O}^{*}}(x, y, z)=x^{3} y+y^{3}+z^{2}=0 \quad \text { in } \quad \mathbb{C} P^{2}(4,6,9) .
$$

$\boldsymbol{E}_{\mathbf{8}}$. In this final case $\Gamma=\mathbb{I}^{*}$ is the binary icosahedral group which is a double cover of the simple icosahedral group $\Gamma_{0}=\mathbb{I} \cong A_{5}$ of order 60 , and $S^{3} / \mathbb{I}^{*}$ is the Poincaré homology sphere $L(5,3,2)$. It has a presentation in terms of generators $\xi_{1}, \xi_{2}, \xi_{3}$ with relations

$$
\xi_{1}^{2}=\xi_{2}^{3}=\xi_{3}^{5}=\xi_{1} \xi_{2} \xi_{3} .
$$

Once more the order two center of $\mathbb{I}^{*}$ is generated by $h=\xi_{1} \xi_{2} \xi_{3}$, and the branching indices on $\mathbb{C} P^{1} / \mathbb{I}$ are given by the Platonic triple $(2,3,5)$; whence there are three exceptional fibres $S^{1} / \mathbb{Z}_{2}$, $S^{1} / \mathbb{Z}_{3}$, and $S^{1} / \mathbb{Z}_{5}$ each of isotropy one. The line V-bundle $\mathcal{L}_{\mathbb{I}^{*}} \rightarrow \mathbb{C} P^{1} / \mathbb{I}$ with $\left|\mathcal{L}_{\mathbb{I}^{*}}\right|=\mathcal{L}$ has orbifold Chern class

$$
c_{1}\left(\mathcal{L}_{\mathbb{T}^{*}}\right)=\frac{61}{30} .
$$

Note that the orbifold Chern class is always of the form $c_{1}\left(\mathcal{L}_{\Gamma}\right)=1+\frac{2}{\mid \Gamma_{0}}$.

The extended simply laced Dynkin diagram $E_{8}$ for the affine exceptional $\widehat{E}_{8}$ Lie algebra is

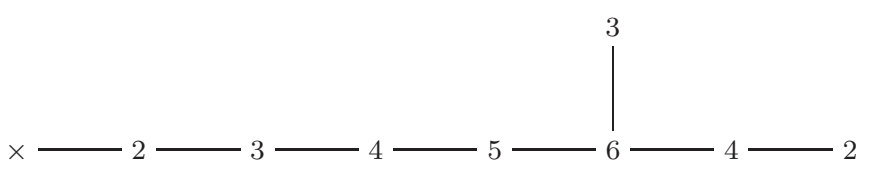

Similarly to the $E_{6}$ and $E_{7}$ cases, the finite subgroup $\mathbb{I}^{*} \subset \mathrm{SU}(2)$ is generated by $\mathbb{D}_{5}^{*}$ and an additional element of $\mathrm{SU}(2)$. However, it is more convenient to notice that it is also generated by

$$
\left(\sigma_{\beta}^{\alpha}\right)=-\left(\begin{array}{cc}
\zeta_{5}^{-2} & 0 \\
0 & \zeta_{5}^{2}
\end{array}\right) \quad \text { and } \quad\left(\widetilde{\sigma}_{\beta}^{\alpha}\right)=\frac{1}{\zeta_{5}^{2}-\zeta_{5}^{-2}}\left(\begin{array}{cc}
\zeta_{5}+\zeta_{5}^{-1} & 1 \\
1 & -\zeta_{5}-\zeta_{5}^{-1}
\end{array}\right) \text {. }
$$

The additional transformations by $\widetilde{\sigma}$ in $(2.30)$ are given by

$$
\begin{gathered}
\pi(\widetilde{\sigma})\left(X_{1}\right)=\frac{1}{5}\left(\zeta_{5}^{2}-\zeta_{5}^{-2}\right)\left(\zeta_{5}-\zeta_{5}^{-1}\right)\left(X_{1}-2 X_{3}\right), \quad \pi(\widetilde{\sigma})\left(X_{2}\right)=-\frac{\left(\zeta_{5}^{2}-\zeta_{5}^{-2}\right)^{2}}{3+\zeta_{5}^{2}+\zeta_{5}^{-2}} X_{2} \\
\quad \text { and } \quad \pi(\widetilde{\sigma})\left(X_{3}\right)=-\frac{1}{5}\left(\zeta_{5}^{2}-\zeta_{5}^{-2}\right)\left(\zeta_{5}-\zeta_{5}^{-1}\right)\left(2 X_{1}+X_{3}\right) .
\end{gathered}
$$

Note that, in contrast to the $A_{k}$ and $D_{k}$ cases, for the $E$-series the $\Gamma$-action generally mixes horizontal and vertical components of one-forms on the $S^{1}$-bundle (2.6). The Poincaré three-sphere $S^{3} / \mathbb{I}^{*}$ also has a presentation as the weighted projective curve

$$
f_{\mathbb{I}^{*}}(x, y, z)=x^{5}+y^{3}+z^{2}=0 \quad \text { in } \quad \mathbb{C} P^{2}(6,10,15) .
$$




\section{Homogeneous vector bundles and quiver representations}

$\Gamma$-modules. As previously, denote by $V_{\ell} \cong \mathbb{C}^{n_{\ell}}$ for $\ell=0,1, \ldots, r_{\Gamma}$ the irreducible unitary representations of the ADE orbifold group $\Gamma$, and introduce the vector space

$$
\widehat{V}=\bigoplus_{\ell=0}^{r_{\Gamma}} V_{\ell}
$$

which is the multiplicity space for the regular representation of $\Gamma$ of dimension $|\Gamma|$, i.e. the finitedimensional vector space of functions on $\Gamma$. The action of a group element $g \in \Gamma$ on $\widehat{V}$ is represented by $n_{\ell} \times n_{\ell}$ unitary matrices $V_{\ell}(g)$ on $V_{\ell}$ for each $\ell=0,1, \ldots, r_{\Gamma}$. Let us fix the vector space

$$
V_{R}=\bigoplus_{\ell=0}^{r_{\Gamma}} R_{\ell} \otimes V_{\ell} \cong \mathbb{C}^{N} \quad \text { with } \quad R_{\ell} \cong \mathbb{C}^{N_{\ell}} \quad \text { and } \quad N:=\sum_{\ell=0}^{r_{\Gamma}} n_{\ell} N_{\ell} .
$$

Every $\Gamma$-module is of this form: The action of $\Gamma$ on (3.2) is defined by a group homomorphism $\gamma: \Gamma \rightarrow \mathrm{U}(N)$ with

$$
w^{\ell} \otimes v_{\ell} \longmapsto \gamma(g)\left(w^{\ell} \otimes v_{\ell}\right)=w^{\ell} \otimes\left(V_{\ell}(g)\left(v_{\ell}\right)\right)
$$

for all $g \in \Gamma, w^{\ell} \in R_{\ell}$ and $v_{\ell} \in V_{\ell}$.

$\Gamma$-projection. For any irreducible representation $V_{\ell}$ of $\Gamma$, the fibred product

$$
\mathcal{V}_{\ell}:=\mathrm{SU}(2) \times_{\Gamma} V_{\ell}
$$

is a homogeneous complex vector bundle of rank $n_{\ell}$ over the Sasaki-Einstein orbifold $S^{3} / \Gamma$. Using (3.4), one can introduce an $\mathrm{SU}(2)$-equivariant complex vector bundle of rank $N$ over $S^{3} / \Gamma$ as the Whitney sum

$$
\bigoplus_{\ell=0}^{r_{\Gamma}} R_{\ell} \otimes \mathcal{V}_{\ell} \quad \text { with } \quad R_{\ell} \cong \mathbb{C}^{N_{\ell}} \quad \text { and } \quad \sum_{\ell=0}^{r_{\Gamma}} n_{\ell} N_{\ell}=N .
$$

Since $\Gamma \subset \mathrm{SU}(2)$, the bundle (3.5) is $\Gamma$-equivariant. The action of $\Gamma$ on the components of any anti-Hermitian connection $X=X_{a} e^{a}$ on the bundle (3.5) is given by a combination of the action (2.30) and the adjoint action generated by (3.3) as

$$
X_{1}+\mathrm{i} X_{2} \longmapsto \gamma(g) \pi(g)\left(X_{1}+\mathrm{i} X_{2}\right) \gamma(g)^{-1} \quad \text { and } \quad X_{3} \longmapsto \gamma(g) \pi(g)\left(X_{3}\right) \gamma(g)^{-1} .
$$

If we consider a connection on a bundle over the Calabi-Yau cone (2.22) then one additionally has the $\Gamma$-action

$$
X_{r} \longmapsto \gamma(g) X_{r} \gamma(g)^{-1}
$$

The $\Gamma$-action on sections of the bundle (3.5) is given by (3.3), while on $\bigoplus_{\ell=0}^{r_{\Gamma}} \mathfrak{u}\left(N_{\ell}\right)$-valued sections $A$ of the corresponding adjoint bundle it is given by

$$
A \longmapsto \gamma(g) A \gamma(g)^{-1} \text {. }
$$

Alternatively, one may start from a complex vector bundle over $S^{3}$ of rank $N$ with gauge group $\mathrm{U}(N)$ broken to the subgroup

$$
\mathcal{G}(R)=\prod_{\ell=0}^{r_{\Gamma}} \mathrm{U}\left(N_{\ell}\right)
$$

commuting with the $\Gamma$-action (3.3), after imposing $\Gamma$-symmetry which reduces the initial bundle to the vector bundle (3.5) over $S^{3} / \Gamma$. 
$\Gamma$-equivariance. After imposing $\Gamma$-symmetry, any homogeneous rank $N$ Hermitian vector bundle over $S^{3}$ decomposes into isotopical components as the bundle (3.5) with the gauge group (3.9) under the action of the orbifold group $\Gamma$. The requirement of $\Gamma$-equivariance of an $\mathrm{SU}(2)$ equivariant connection $X=X_{a} e^{a}$ states that it defines a covariant representation of $\Gamma$, in the sense that its components satisfy the equations

$$
\gamma(g) X_{a} \gamma(g)^{-1}=\pi(g)^{-1}\left(X_{a}\right)
$$

for all $g \in \Gamma$, where $\gamma(g)$ is given in (3.3). This condition decomposes the connection components fibrewise as

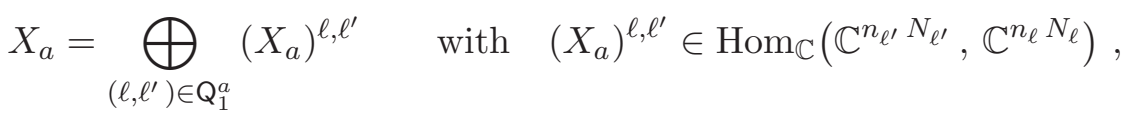

where $\mathrm{Q}_{1}^{a}$ for $a=1,2,3$ is the set of non-zero blocks; given $\ell=0,1, \ldots, r_{\Gamma}$, the corresponding pairs $\left(\ell, \ell^{\prime}\right) \in \mathrm{Q}_{1}^{a}$ are found by comparing (3.3) with the covariance conditions

$$
\gamma(g)\left(X_{a}\left(w^{\ell^{\prime}} \otimes v_{\ell^{\prime}}\right)\right)=\pi(g)^{-1}\left(X_{a}\right)\left(w^{\ell^{\prime}} \otimes\left(V_{\ell}(g)\left(v_{\ell^{\prime}}\right)\right)\right)
$$

for $g \in \Gamma, w^{\ell^{\prime}} \in R_{\ell^{\prime}}$ and $v_{\ell^{\prime}} \in V_{\ell^{\prime}}$.

The requirement of $\Gamma$-equivariance in this way naturally defines a representation of a finite quiver $\mathrm{Q}=\mathrm{Q}_{\Gamma}=\left(\mathrm{Q}_{0}, \mathrm{Q}_{1}, \mathrm{~s}, \mathrm{t}\right)$ associated with the Sasaki-Einstein orbifold $S^{3} / \Gamma$, i.e. an oriented graph given by a finite set of vertices $Q_{0}$, a finite set of arrows $Q_{1} \subset Q_{0} \times Q_{0}$, and two projection maps s, t: $\mathrm{Q}_{1} \rightrightarrows \mathrm{Q}_{0}$ taking each arrow to its source vertex and its target vertex respectively; in the present case the vertices are just the nodes of the affine ADE Dynkin diagram corresponding to $\Gamma$, while the arrows are determined by the non-zero blocks of the horizontal and vertical connection components $X_{1}+\mathrm{i} X_{2}$ and $X_{3}$. A (linear) representation of the quiver $\mathrm{Q}$ is a $\mathrm{Q}_{0}$-graded vector space $R=\bigoplus_{\ell=0}^{r_{\Gamma}} R_{\ell}, R_{\ell} \cong \mathbb{C}^{N_{\ell}}$ together with a collection of linear transformations $B=\left(B_{e}: R_{\mathrm{s}(e)} \rightarrow\right.$ $\left.R_{\mathrm{t}(e)}\right)_{e \in \mathrm{Q}_{1}}$. Given a $\mathrm{Q}_{0}$-graded vector space $R$, the representation space

$$
\operatorname{Rep}_{\mathbf{Q}}(R):=\bigoplus_{e \in Q_{1}} \operatorname{Hom}_{\mathbb{C}}\left(R_{\mathrm{s}(e)}, R_{\mathrm{t}(e)}\right)
$$

is the affine variety parameterizing representations of the quiver $\mathrm{Q}$ into $R$. Note that $R$ is the multiplicity space of the $\Gamma$-module (3.2), and hence there is a one-to-one correspondence between representations of the discrete group $\Gamma \subset \mathrm{SU}(2)$ and the representation varieties (3.13). The gauge group (3.9) acts naturally on (3.13) as

$$
B_{e} \longmapsto g_{\mathrm{t}(e)} B_{e} g_{\mathbf{s}(e)}^{-1}
$$

where $g_{\ell} \in \mathrm{U}\left(N_{\ell}\right)$; note that the diagonal $\mathrm{U}(1)$ subgroup of scalars in (3.9) acts trivially, so we will often factor it out and work instead with the projective gauge group $\mathrm{PG}(R):=\mathcal{G}(R) / \mathrm{U}(1)$. If the quiver is equiped with a set of relations $\mathrm{R}$, i.e. formal $\mathbb{C}$-linear combinations of arrow compositions of the quiver, then we denote by $\operatorname{Rep}_{\mathrm{Q}, \mathrm{R}}(R)$ the subvariety of (3.13) consisting of representations of $\mathrm{Q}$ into $R$ which satisfy the relations $\mathrm{R}$. For background on quivers and their representations in the context of this paper, see e.g. [21, Sect. 5] and [9].

$\boldsymbol{A}_{\boldsymbol{k}}$. In this case $n_{\ell}=1$ for all $\ell=0,1, \ldots, k(\bmod (k+1))$ and the generator $h$ of the cyclic group $\mathbb{Z}_{k+1}$ acts on $V_{\ell} \cong \mathbb{C}$ as $V_{\ell}(h)\left(v_{\ell}\right)=\zeta_{k+1}^{\ell} v_{\ell}$. On the vector space (3.1) the generating element acts as the diagonal matrix

$$
\operatorname{diag}\left(1, \zeta_{k+1}, \ldots, \zeta_{k+1}^{k}\right)
$$


In this case we will also often consider the vector spaces

$$
V_{1}^{\oplus n} \oplus V_{-n} \cong \mathbb{C}^{n+1} \ni\left(z^{1}, \ldots, z^{n}, z^{n+1}\right)
$$

for $n \geq 1$, on which the generator $h$ acts as the map

$$
\left(z^{1}, \ldots, z^{n}, z^{n+1}\right) \longmapsto\left(\zeta_{k+1} z^{1}, \ldots, \zeta_{k+1} z^{n}, \zeta_{k+1}^{-n} z^{n+1}\right)
$$

which defines a homomorphism of the cyclic group $\mathbb{Z}_{k+1}$ into the Lie group $\mathrm{SU}(n+1)$.

The covariant representations of $\mathbb{Z}_{k+1}$ are characterized by the equations

$$
\gamma(h)\left(X_{1}+\mathrm{i} X_{2}\right) \gamma(h)^{-1}=\zeta_{k+1}^{-2}\left(X_{1}+\mathrm{i} X_{2}\right) \quad \text { and } \quad \gamma(h) X_{3} \gamma(h)^{-1}=X_{3},
$$

and for $k \geq 2$ it is easy to see that the non-zero blocks are given fibrewise by the matrix elements

$$
\begin{aligned}
\left(X_{1}+\mathrm{i} X_{2}\right)^{\ell, \ell+2} & =: \quad \varphi_{\ell} \in \operatorname{Hom}_{\mathbb{C}}\left(\mathbb{C}^{N_{\ell+2}}, \mathbb{C}^{N_{\ell}}\right), \\
\left(X_{1}-\mathrm{i} X_{2}\right)^{\ell+2, \ell} & =: \quad-\varphi_{\ell}^{\dagger} \in \operatorname{Hom}_{\mathbb{C}}\left(\mathbb{C}^{N_{\ell}}, \mathbb{C}^{N_{\ell+2}}\right), \\
\left(X_{3}\right)^{\ell, \ell} & =: \quad \chi_{\ell} \in \operatorname{End}_{\mathbb{C}}\left(\mathbb{C}^{N_{\ell}}\right)
\end{aligned}
$$

for $\ell=0,1, \ldots, k$, where we used the relation

$$
X_{1}-\mathrm{i} X_{2}=-\left(X_{1}+\mathrm{i} X_{2}\right)^{\dagger} .
$$

In the case $k=1$, when $\zeta_{2}^{2}=1$ and the $\mathbb{Z}_{2}$-projection is given by $S^{3} \rightarrow \mathbb{R} P^{3}$, one has only nonvanishing blocks $\left(X_{1} \pm \mathrm{i} X_{2}\right)^{\ell, \ell},\left(X_{3}\right)^{\ell, \ell} \in \operatorname{End}_{\mathbb{C}}\left(\mathbb{C}^{N_{\ell}}\right)$ for $\ell=0,1$. Analysis of the explicit form of the matrices (3.19) and of the corresponding quivers shows that the general cases of even and odd rank $k$ should be treated separately.

a) $\underline{k=2 q, S^{3} / \mathbb{Z}_{2 q+1} \text { : }}$

Using the property $\zeta_{k+1}^{2 q+1}=1$, one can show that the matrix

$$
\operatorname{diag}\left(1, \zeta_{k+1}^{2}, \ldots, \zeta_{k+1}^{2 k}\right)=\operatorname{diag}\left(1, \zeta_{k+1}^{2}, \ldots, \zeta_{k+1}^{2 q}, \zeta_{k+1}, \zeta_{k+1}^{3}, \ldots, \zeta_{k+1}^{2 q-1}\right)
$$

is equivalent to the matrix (3.15) with permuted diagonal elements. Then by using the matrix

$\gamma(h)=\operatorname{diag}\left(\mathbf{1}_{\mathbb{C}^{N_{0}}} \otimes 1, \mathbf{1}_{\mathbb{C}^{N_{1}}} \otimes \zeta_{k+1}^{2}, \ldots, \mathbf{1}_{\mathbb{C}^{N_{q}}} \otimes \zeta_{k+1}^{2 q}, \mathbf{1}_{\mathbb{C}^{N_{q+1}}} \otimes \zeta_{k+1}, \mathbf{1}_{\mathbb{C}^{N_{q}+2}} \otimes \zeta_{k+1}^{3}, \ldots, \mathbf{1}_{\mathbb{C}^{N_{2 q}}} \otimes \zeta_{k+1}^{2 q-1}\right)$

in (3.18) we obtain the solution

$$
\begin{aligned}
& \left(X_{1}+\mathrm{i} X_{2}\right)^{\ell, \ell+1}=: \quad \phi_{\ell+1} \in \operatorname{Hom}_{\mathbb{C}}\left(\mathbb{C}^{N_{\ell+1}}, \mathbb{C}^{N_{\ell}}\right) \quad \text { and } \quad\left(X_{1}+\mathrm{i} X_{2}\right)^{k, 0}=: \phi_{k+1},(3 \\
& \left(X_{1}-\mathrm{i} X_{2}\right)^{\ell+1, \ell}=: \quad-\phi_{\ell+1}^{\dagger} \in \operatorname{Hom}_{\mathbb{C}}\left(\mathbb{C}^{N_{\ell}}, \mathbb{C}^{N_{\ell+1}}\right) \quad \text { and } \quad\left(X_{1}-\mathrm{i} X_{2}\right)^{0, k}=:-\phi_{k+1}^{\dagger}
\end{aligned}
$$

with $\ell=0,1, \ldots, 2 q-1$, and

$$
\left(X_{3}\right)^{\ell, \ell}=: \varrho_{\ell} \in \operatorname{End}_{\mathbb{C}}\left(\mathbb{C}^{N_{\ell}}\right)
$$

with $\ell=0,1, \ldots, 2 q$, where $\varrho_{\ell}^{\dagger}=-\varrho_{\ell}$. Note that in these equations we use the same symbol $N_{\ell}$ as in (3.19), but they are in fact related by permutation, as are $\chi_{\ell}$ and $\varrho_{\ell}$, and $\varphi_{\ell}$ and $\phi_{\ell}$. Finally we obtain the irreducible affine $\widehat{A}_{2 q}$-type quivers $\mathrm{Q}_{\widehat{A}_{2 q}}$ given by 


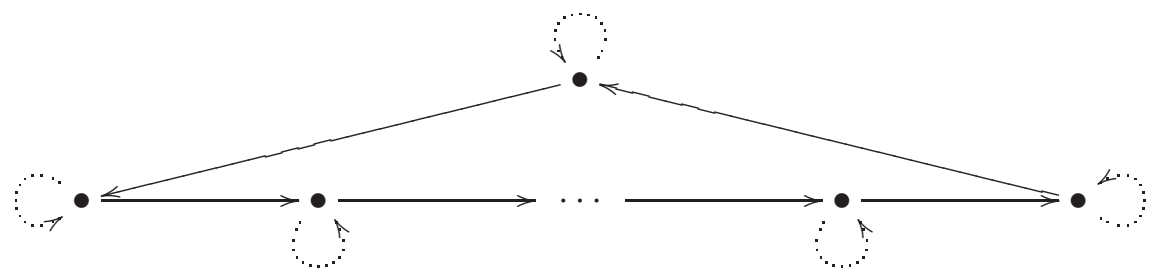

with $2 q+1$ vertices, arrows and loop edges. For clarity, throughout we designate arrows associated to the horizontal components $X_{1}+\mathrm{i} X_{2}$ by solid lines and arrows associated to the vertical components $X_{3}$ with dashed lines; in particular, here the loop edges are associated with the bundle endomorphisms $\varrho_{\ell}$. The underlying graph of this quiver is the extended affine Dynkin diagram $A_{2 q}$ from (2.35).

b) $\underline{k=2 q+1, S^{3} / \mathbb{Z}_{2 q+2} \text { : }}$

In this case one finds that the $\mathbb{Z}_{2 q+2}$-equivariant vector bundle over $S^{3} / \mathbb{Z}_{2 q+2}$ is a direct sum of two irreducible bundles and the associated quiver splits into two connected quivers of the type (3.25). Arguing in a similar way as above, we now have

$\gamma(h)=\operatorname{diag}\left(\mathbf{1}_{\mathbb{C}^{N_{0}}} \otimes 1, \mathbf{1}_{\mathbb{C}^{N_{1}}} \otimes \zeta_{k+1}^{2}, \ldots, \mathbf{1}_{\mathbb{C}^{N_{q}}} \otimes \zeta_{k+1}^{2 q}, \mathbf{1}_{\mathbb{C}^{N_{q+1}}} \otimes 1, \mathbf{1}_{\mathbb{C}^{N_{q+2}}} \otimes \zeta_{k+1}^{2}, \ldots, \mathbf{1}_{\mathbb{C}^{N_{2 q+1}}} \otimes \zeta_{k+1}^{2 q}\right)$

with $\zeta_{k+1}^{2 \ell}=\zeta_{q+1}^{\ell}$, and the corresponding reduced quivers are given by

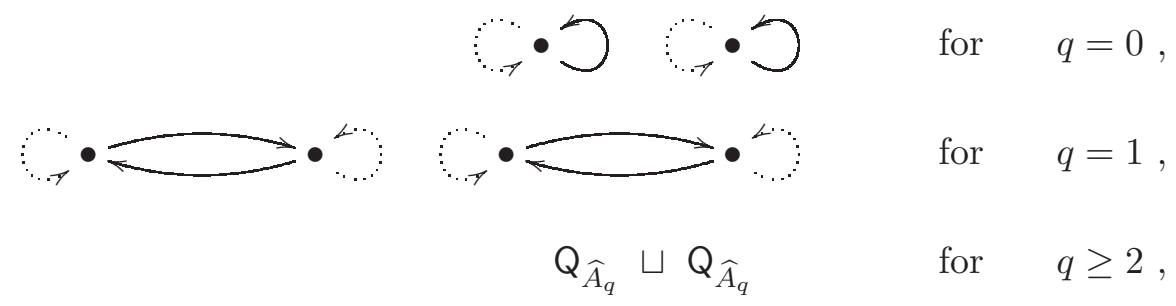

with the quiver $(3.29)$ having $(q+1)+(q+1)$ vertices, arrows and loop edges.

$\boldsymbol{D}_{\boldsymbol{k}}$. The dicyclic group $\mathbb{D}_{k-2}^{*}$ has $k-1$ two-dimensional representations $W_{j} \cong \mathbb{C}^{2}$ on which the generators $\xi_{2}$ and $\xi_{3}$ act as the matrices

$$
W_{j}\left(\xi_{2}\right)=\left(\begin{array}{cc}
0 & 1 \\
(-1)^{j} & 0
\end{array}\right) \quad \text { and } \quad W_{j}\left(\xi_{3}\right)=\left(\begin{array}{cc}
\zeta_{2(k-2)}^{j} & 0 \\
0 & \zeta_{2(k-2)}^{-j}
\end{array}\right)
$$

for $j=0,1, \ldots, k-2$; in particular, $W_{1}$ is the fundamental representation $(2.46)$. For $\ell=1, \ldots, k-3$ the representations $V_{\ell}:=W_{\ell}$ are irreducible, while $W_{0}=V_{0} \oplus V_{k}$ and $W_{k-2}=V_{k-2} \oplus V_{k-1}$ simultaneously diagonalize into two eigenlines, with $V_{0}$ the trivial representation and

$$
V_{k}\left(\xi_{2}\right)=V_{k-2}\left(\xi_{3}\right)=V_{k-1}\left(\xi_{3}\right)=-1=-V_{k}\left(\xi_{3}\right) \quad \text { and } \quad V_{k-2}\left(\xi_{2}\right)=-\mathrm{i}^{k}=-V_{k-1}\left(\xi_{2}\right) .
$$

The covariant representations of $\mathbb{D}_{k-2}^{*}$ are characterized by the equations

$$
\gamma\left(\xi_{2}\right)\left(X_{1}+\mathrm{i} X_{2}\right) \gamma\left(\xi_{2}\right)^{-1}=\left(X_{1}+\mathrm{i} X_{2}\right)^{\dagger} \quad \text { and } \quad \gamma\left(\xi_{2}\right) X_{3} \gamma\left(\xi_{2}\right)^{-1}=-X_{3}
$$

together with

$$
\gamma\left(\xi_{3}\right)\left(X_{1}+\mathrm{i} X_{2}\right) \gamma\left(\xi_{3}\right)^{-1}=\zeta_{2(k-2)}^{-2}\left(X_{1}+\mathrm{i} X_{2}\right) \quad \text { and } \quad \gamma\left(\xi_{3}\right) X_{3} \gamma\left(\xi_{3}\right)^{-1}=X_{3},
$$


where we have used the relation (3.20). By working in the canonical basis of $W_{j} \cong \mathbb{C}^{2}$, from these equations it is straightforward to see that all representation spaces $W_{j}$ for $j=0,1, \ldots, k-2$ are $X_{3}$-invariant, while under the horizontal connection components they transform as

$$
\left(X_{1}+\mathrm{i} X_{2}\right)\left(W_{0}\right) \subset W_{2} \quad \text { and } \quad\left(X_{1}+\mathrm{i} X_{2}\right)\left(W_{1}\right) \subset W_{3}
$$

and

$$
\left(X_{1}+\mathrm{i} X_{2}\right)\left(W_{k-3}\right) \subset W_{k-5} \quad \text { and } \quad\left(X_{1}+\mathrm{i} X_{2}\right)\left(W_{k-2}\right) \subset W_{k-4},
$$

together with

$$
\left(X_{1}+\mathrm{i} X_{2}\right)\left(W_{j}\right) \subset W_{j-2} \oplus W_{j+2} \quad \text { for } \quad j=2, \ldots, k-4 .
$$

After diagonalising the reducible representations $W_{0}$ and $W_{k-2}$ into their simultaneous eigenlines, some straightforward linear algebra shows that $\left(X_{1}+\mathrm{i} X_{2}\right)\left(V_{\ell}\right) \subset V_{2}$ for $\ell=0, k$ and $\left(X_{1}+\right.$ i $\left.X_{2}\right)\left(V_{\ell}\right) \subset V_{k-4}$ for $\ell=k-2, k-1$, while $X_{3}\left(V_{0}\right) \subset V_{k}, X_{3}\left(V_{k}\right) \subset V_{0}$ and $X_{3}\left(V_{k-2}\right) \subset V_{k-1}$, $X_{3}\left(V_{k-1}\right) \subset V_{k-2}$.

Following our treatment of the $A_{2 q+1}$ family above, we use the block diagonal matrices

$$
\gamma\left(\xi_{j}\right)=\operatorname{diag}\left(\mathbf{1}_{\mathbb{C}^{N_{0}}} \oplus \mathbf{1}_{\mathbb{C}^{N_{k}}}, \mathbf{1}_{\mathbb{C}^{N_{1}}} \otimes W_{2}\left(\xi_{j}\right), \ldots, \mathbf{1}_{\mathbb{C}^{N_{k-3}}} \otimes W_{2(k-3)}\left(\xi_{j}\right), \mathbf{1}_{\mathbb{C}^{N_{k-2}}} \oplus \mathbf{1}_{\mathbb{C}^{N_{k-1}}}\right)
$$

in (3.32) and (3.33) to obtain the solution

$$
\begin{aligned}
& \left(X_{1}+\mathrm{i} X_{2}\right)^{\ell, \ell-1}=: \phi_{\ell-1}^{+} \in \operatorname{Hom}_{\mathbb{C}}\left(\mathbb{C}^{2 N_{\ell-1}}, \mathbb{C}^{2 N_{\ell}}\right), \\
& \left(X_{1}-\mathrm{i} X_{2}\right)^{\ell-1, \ell}=: \quad-\phi_{\ell-1}^{+} \dagger^{\dagger} \in \operatorname{Hom}_{\mathbb{C}}\left(\mathbb{C}^{2 N_{\ell}}, \mathbb{C}^{2 N_{\ell-1}}\right), \\
& \left(X_{1}+\mathrm{i} X_{2}\right)^{\ell, \ell+1}=: \phi_{\ell+1}^{-} \in \operatorname{Hom}_{\mathbb{C}}\left(\mathbb{C}^{2 N_{\ell+1}}, \mathbb{C}^{2 N_{\ell}}\right), \\
& \left(X_{1}-\mathrm{i} X_{2}\right)^{\ell+1, \ell}=: \quad-\phi_{\ell+1}^{-} \dagger \in \operatorname{Hom}_{\mathbb{C}}\left(\mathbb{C}^{2 N_{\ell}}, \mathbb{C}^{2 N_{\ell+1}}\right)
\end{aligned}
$$

for $\ell=1, \ldots, k-4$, together with

$$
\begin{aligned}
& \left(X_{1}+\mathrm{i} X_{2}\right)^{\ell^{\prime}, \ell}=: \quad \varphi_{\ell} \in \operatorname{Hom}_{\mathbb{C}}\left(\mathbb{C}^{N_{\ell}}, \mathbb{C}^{2 N_{\ell^{\prime}}}\right), \\
& \left(X_{1}-\mathrm{i} X_{2}\right)^{\ell, \ell^{\prime}}=:-\varphi_{\ell}^{\dagger} \in \operatorname{Hom}_{\mathbb{C}}\left(\mathbb{C}^{2 N_{\ell^{\prime}}}, \mathbb{C}^{N_{\ell}}\right),
\end{aligned}
$$

for $\ell^{\prime}=1$ (resp. $\ell^{\prime}=k-3$ ) and $\ell=0, k$ (resp. $\ell=k-2, k-1$ ), while for the vertical components we find

$$
\begin{aligned}
\left(X_{3}\right)^{\ell, \ell} & =: \quad \varrho_{\ell}=-\varrho_{\ell}^{\dagger} \in \operatorname{End}_{\mathbb{C}}\left(\mathbb{C}^{2 N_{\ell}}\right), \\
\left(X_{3}\right)^{\ell^{\prime}, \ell^{\prime \prime}} & =: \quad \chi_{\ell^{\prime}} \in \operatorname{Hom}_{\mathbb{C}}\left(\mathbb{C}^{N_{\ell^{\prime \prime}}}, \mathbb{C}^{N_{\ell^{\prime}}}\right), \\
\left(X_{3}\right)^{\ell^{\prime \prime}, \ell^{\prime}} & =:-\chi_{\ell^{\prime}}^{\dagger} \in \operatorname{Hom}_{\mathbb{C}}\left(\mathbb{C}^{N_{\ell^{\prime}}}, \mathbb{C}^{N_{\ell^{\prime \prime}}}\right)
\end{aligned}
$$

for $\ell=1, \ldots, k-3$, and $\ell^{\prime}=k$ (resp. $\left.\ell^{\prime \prime}=0\right)$ and $\ell^{\prime}=k-1$ (resp. $\left.\ell^{\prime \prime}=k-2\right)$. We thereby arrive at the quiver

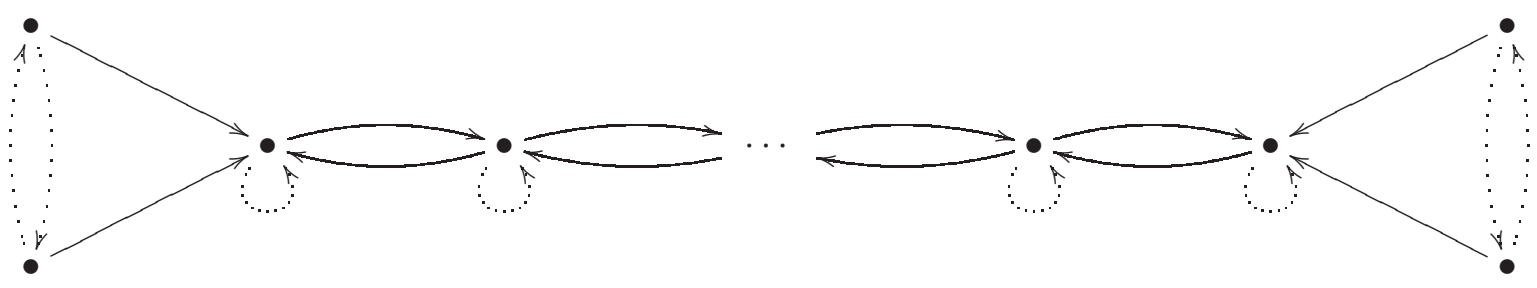


with $k+1$ vertices, $2 k$ arrows and $k-3$ loop edges; its underlying graph is the affine Dynkin diagram $\mathrm{D}_{k}$ from (2.45). Note that the horizontal segment of this quiver consists of a chain of $k-4$ connected $\widehat{A}_{1}$-type quivers from (3.28).

$\boldsymbol{E}_{\boldsymbol{k}}$. The constructions above can in principle be extended to the exceptional series. For example, the binary tetrahedral group $\mathbb{T}^{*}$ has seven irreducible representations consisting of three one-dimensional representations given by the quotient $\mathbb{T}^{*} \rightarrow \mathbb{Z}_{3}$, three two-dimensional representations obtained by taking tensor products of these one-dimensional representations with the fundamental representation of $\mathbb{T}^{*} \subset \mathrm{SU}(2)$, and one three-dimensional representation given by the quotient $\mathbb{T}^{*} \rightarrow \mathbb{T} \subset \mathrm{SO}(3)$; we leave it to the interested reader to work out the details of the corresponding $\mathrm{E}_{6}$ quiver diagram. For the extended Dynkin diagrams $\mathrm{E}_{7}$ and $\mathrm{E}_{8}$ the representation theory becomes somewhat more complicated.

\section{Equivariant dimensional reduction and quiver bundles}

Equivariant vector bundles. In this section we consider the dimensional reduction of invariant connections on equivariant vector bundles over product manifolds. Let $\mathcal{E}$ be an $\mathrm{SU}(2)$-equivariant Hermitian vector bundle of $\operatorname{rank} N$ over $M \times S^{3}$, where $M$ is a smooth, closed and oriented manifold of real dimension $D$; the group $\mathrm{SU}(2)$ acts trivially on $M$ and by isometries on $S^{3} \cong \mathrm{SU}(2)$. The sphere $S^{3}$ can be regarded as a coset space $G / H$ with $G=\mathrm{SU}(2)$ and the trivial stabilizer subgroup $H=\{1\}$; for the relevant background on equivariant dimensional reduction over coset spaces, see e.g. $[1,2,3]$.

We will use the same symbol $\mathcal{E}$ for the vector bundle

$$
\mathcal{E} \longrightarrow M \times S^{3} / \Gamma
$$

obtained by projection to the orbifold $M \times S^{3} / \Gamma$. By standard induction and reduction, there is an equivalence between $\mathrm{SU}(2)$-equivariant vector bundles (4.1) and $\Gamma$-equivariant vector bundles over $M$ which are described by the quivers $Q_{\Gamma}$ from Sect. 3 ; the finite orbifold group $\Gamma \subset \mathrm{SU}(2)$ also acts trivially on $M$. A representation of the quiver $\mathrm{Q}_{\Gamma}$ in the category $\operatorname{Bun}(M)$ of complex vector bundles on $M$ is called a quiver bundle on $M$.

Every SU(2)-equivariant complex vector bundle (4.1) can be decomposed uniquely up to isomorphism into isotopical components as a Whitney sum

$$
\mathcal{E}=\bigoplus_{\ell=0}^{r_{\Gamma}} E_{\ell} \otimes \mathcal{V}_{\ell}
$$

where $E_{\ell} \rightarrow M$ are Hermitian vector bundles of rank $N_{\ell}$ with $\sum_{\ell=0}^{r_{\Gamma}} n_{\ell} N_{\ell}=N$ and trivial $\Gamma$-action, and the homogeneous bundles $\mathcal{V}_{\ell} \rightarrow S^{3} / \Gamma$ are defined in (3.4). As we showed in Sect. 3, the gauge group $\mathcal{G}(R)$ of the bundle (4.2) is given by (3.9).

$\Gamma$-equivariant connections. Let $\mathcal{A}$ be an $\mathrm{SU}(2)$-equivariant gauge connection on $\mathcal{E}$ and $\mathcal{F}=\mathrm{d} \mathcal{A}+\mathcal{A} \wedge \mathcal{A}$ its curvature, both with values in the Lie algebra $\mathfrak{u}(N)$. It has the form

$$
\mathcal{A}=A+X=A_{\hat{\mu}} e^{\hat{\mu}}+X_{a} e^{a},
$$

where $e^{\hat{\mu}}$ and $e^{a}$ are basis one-forms on $M$ and $S^{3}$, respectively, and $A_{\hat{\mu}}$ and $X_{a}$ are $\mathfrak{u}(N)$-valued matrices which depend only on the coordinates of $M$ with $\hat{\mu}, \hat{\nu}, \ldots=1, \ldots, D$. Since $S^{3}$ is a group manifold, there are no further restrictions on $A_{\hat{\mu}}$ and $X_{a}$ coming from $\mathrm{SU}(2)$-invariance. 
We shall also use the same symbol $\mathcal{A}$ for the connection obtained by projecting (4.3) to the orbifold $M \times S^{3} / \Gamma$. The gauge potential projection from $M \times S^{3}$ to $M \times S^{3} / \Gamma$ is defined by the equations

$$
\gamma(g) A_{\hat{\mu}} \gamma(g)^{-1}=A_{\hat{\mu}}
$$

for all $g \in \Gamma$, together with the equations (3.10) for $X_{a}$ which are resolved by the matrices (3.11).

The calculation of the curvature $\mathcal{F}=\mathrm{d} \mathcal{A}+\mathcal{A} \wedge \mathcal{A}$ for $\mathcal{A}$ of the form (4.3) yields

$$
\mathcal{F}=F+\left(\mathrm{d} X_{a}+\left[A, X_{a}\right]\right) \wedge e^{a}+\frac{1}{2}\left(\left[X_{a}, X_{b}\right]-2 \varepsilon_{a b}^{c} X_{c}\right) e^{a} \wedge e^{b},
$$

where $F=\mathrm{d} A+A \wedge A$ is the curvature of the gauge potential $A$ on $M$ with gauge group $\mathcal{G}(R)$. Given local real coordinates $x^{\hat{\mu}}$ on $M$ one can choose $\mathrm{d} x^{\hat{\mu}}$ as basis one-forms $e^{\hat{\mu}}$ on $M$. Then from (4.5) we find the non-vanishing components of the field strength tensor

$$
\begin{aligned}
\mathcal{F}_{\hat{\mu} \hat{\nu}} & =\partial_{\hat{\mu}} A_{\hat{\nu}}-\partial_{\hat{\nu}} A_{\hat{\mu}}+\left[A_{\hat{\mu}}, A_{\hat{\nu}}\right], \\
\mathcal{F}_{\hat{\mu} a} & =: \quad D_{\hat{\mu}} X_{a}=\partial_{\hat{\mu}} X_{a}+\left[A_{\hat{\mu}}, X_{a}\right], \\
\mathcal{F}_{a b} & =\left[X_{a}, X_{b}\right]-2 \varepsilon_{a b}^{c} X_{c} .
\end{aligned}
$$

Quiver gauge theory. The dimensional reduction of the Yang-Mills equations on $M \times S^{3} / \Gamma$ can be seen at the level of the Yang-Mills Lagrangian; reduction of the Yang-Mills action functional defines a quiver gauge theory on $M$ associated to the quiver $\mathrm{Q}_{\Gamma}$. Let $\mathrm{d}_{\operatorname{vol}_{M}}$ be the Riemannian volume form with respect to an arbitrarily chosen metric on the manifold $M$, and let $\mathrm{d} \operatorname{vol}_{S^{3} / \Gamma}$ denote the Riemannian volume form associated to the metric (2.16) on the Sasaki-Einstein manifold $S^{3} / \Gamma$; the corresponding Hodge duality operator for the product metric on $M \times S^{3} / \Gamma$ is denoted $\star$. With $\operatorname{tr}_{N}$ denoting the trace in the fundamental representation of the $\mathrm{U}(N)$ gauge group, by substituting in (4.6)-(4.8) and integrating over $S^{3} / \Gamma$ we arrive at the action

$$
\begin{aligned}
S_{\mathrm{YM}}:= & -\frac{1}{4} \int_{M \times S^{3} / \Gamma} \operatorname{tr}_{N} \mathcal{F} \wedge \star \mathcal{F} \\
= & -\frac{1}{8} \int_{M \times S^{3} / \Gamma} \mathrm{d} \mathrm{vol}_{M} \wedge \mathrm{d}_{\operatorname{vol}_{S^{3} / \Gamma}} \operatorname{tr}_{N}\left(\mathcal{F}_{\hat{\mu} \hat{\nu}} \mathcal{F}^{\hat{\mu} \hat{\nu}}+2 \mathcal{F}_{\hat{\mu} a} \mathcal{F}^{\hat{\mu} a}+\mathcal{F}_{a b} \mathcal{F}^{a b}\right) \\
= & -\frac{\pi r^{3}}{6 d} c_{1}\left(\mathcal{L}_{\Gamma}\right) \int_{M} \mathrm{~d} \mathrm{vol}_{M} \operatorname{tr}_{N}\left(F_{\hat{\mu} \hat{\nu}} F^{\hat{\mu} \hat{\nu}}+\frac{2}{r^{2}} D_{\hat{\mu}} X_{a} D^{\hat{\mu}} X_{a}\right. \\
& \left.+\frac{1}{r^{4}} \sum_{a, b=1}^{3}\left(\left[X_{a}, X_{b}\right]-2 \varepsilon_{a b}^{c} X_{c}\right)^{2}\right) .
\end{aligned}
$$

In the sector of this field theory with $A_{\hat{\mu}}=0$ and locally translationally-invariant scalar fields $X_{a}$, the global minima of the action are described by the matrix equations

$$
\mathcal{F}_{a b}=\left[X_{a}, X_{b}\right]-2 \varepsilon_{a b}^{c} X_{c}=0 .
$$

In general these equations will contain both holomorphic F-term constraints on the scalar fields, which define the set of relations $\mathrm{R}_{\Gamma}$ among the arrows for the quiver $\mathrm{Q}_{\Gamma}$, and also non-holomorphic D-term constraints, which yield stability conditions for the corresponding moduli variety of quiver representations. Hence solutions of the BPS equations are determined by stable representations of the quiver with relations $\left(\mathrm{Q}_{\Gamma}, \mathrm{R}_{\Gamma}\right)$. The corresponding stable quotient $\operatorname{Rep}_{\mathrm{Q}_{\Gamma}, \mathrm{R}_{\Gamma}}(R) / / \mathrm{PG}(R)$ is a finite set whose points are in one-to-one correspondence with representations of the Lie algebra $\mathfrak{s u}(2)$ in $\operatorname{Rep}_{Q_{\Gamma}}(R) \subset \mathfrak{u}(N)$. 
$\boldsymbol{A}_{\boldsymbol{k}}$. In this case from (3.22), (3.26) and (4.4) we obtain a block diagonal form similar to $X_{3}$ for the gauge potential $A=A_{\hat{\mu}} e^{\hat{\mu}}$ given by

$$
A=\operatorname{diag}\left(A^{0}, A^{1}, \ldots, A^{k}\right) .
$$

After projection from $M \times S^{3}$ to $M \times S^{3} / \mathbb{Z}_{k+1}$ the scalar field $X=X_{a} e^{a}$ from (4.3) has horizontal components

$$
X_{1}+\mathrm{i} X_{2}=\left(\begin{array}{ccccc}
0 & \phi_{1} & 0 & \cdots & 0 \\
0 & 0 & \phi_{2} & \ddots & \vdots \\
\vdots & \vdots & \ddots & \ddots & 0 \\
0 & 0 & \cdots & 0 & \phi_{k} \\
\phi_{k+1} & 0 & \cdots & 0 & 0
\end{array}\right) \quad \text { and } \quad X_{1}-\mathrm{i} X_{2}=-\left(X_{1}+\mathrm{i} X_{2}\right)^{\dagger}
$$

and vertical component

$$
X_{3}=\operatorname{diag}\left(\varrho_{0}, \varrho_{1}, \ldots, \varrho_{k}\right)
$$

for $k=2 q$. We will not consider here the case $k=2 q+1$ given by the formulas (3.26)-(3.29), as it can be simply reduced to a doubling of the matrices (4.12)-(4.13) with $k$ substituted by $q$.

From (4.11)-(4.13) one sees that the $\mathfrak{u}(N)$-valued gauge potential $\mathcal{A}$ splits into $N_{\ell} \times N_{\ell^{\prime}}$ blocks $\mathcal{A}^{\ell \ell^{\prime}}$ as

$$
\mathcal{A}=\left(\mathcal{A}^{\ell \ell^{\prime}}\right) \quad \text { with } \quad \mathcal{A}^{\ell \ell^{\prime}} \in \operatorname{Hom} \mathbb{C}\left(\mathbb{C}^{N_{\ell^{\prime}}}, \mathbb{C}^{N_{\ell}}\right)
$$

where the indices $\ell, \ell^{\prime}, \ldots$ run over $0,1, \ldots, k$, and

$$
\begin{aligned}
\mathcal{A}^{\ell \ell} & =A^{\ell} \otimes 1+\varrho_{\ell} \otimes e^{3}, \\
\mathcal{A}^{\ell \ell+1} & =: \quad \Phi_{\ell+1}=\frac{1}{2} \phi_{\ell+1} \otimes\left(e^{1}-\mathrm{i} e^{2}\right), \quad \mathcal{A}^{k 0}=: \Phi_{k+1}=\frac{1}{2} \phi_{k+1} \otimes\left(e^{1}-\mathrm{i} e^{2}\right), \\
\mathcal{A}^{\ell+1 \ell} & =-\Phi_{\ell+1}^{\dagger}=-\frac{1}{2} \phi_{\ell+1}^{\dagger} \otimes\left(e^{1}+\mathrm{i} e^{2}\right), \quad \mathcal{A}^{0 k}=-\Phi_{k+1}^{\dagger}=-\frac{1}{2} \phi_{k+1}^{\dagger} \otimes\left(e^{1}+\mathrm{i} e^{2}\right),
\end{aligned}
$$

with all other components vanishing.

For the curvature

$$
\mathcal{F}=\left(\mathcal{F}^{\ell \ell^{\prime}}\right) \quad \text { with } \quad \mathcal{F}^{\ell \ell^{\prime}}=\mathrm{d} \mathcal{A}^{\ell \ell^{\prime}}+\sum_{\ell^{\prime \prime}=0}^{k} \mathcal{A}^{\ell \ell^{\prime \prime}} \wedge \mathcal{A}^{\ell^{\prime \prime} \ell},
$$

we obtain the non-vanishing field strength components

$$
\begin{aligned}
\mathcal{F}^{\ell \ell} & =F^{\ell}-\frac{1}{4}\left(2 \mathrm{i} \varrho_{\ell}+\phi_{\ell}^{\dagger} \phi_{\ell}-\phi_{\ell+1} \phi_{\ell+1}^{\dagger}\right) \beta \wedge \bar{\beta}+\mathrm{d} \varrho_{\ell} \wedge e^{3}, \\
\mathcal{F}^{\ell \ell+1} & =\frac{1}{2} D \phi_{\ell+1} \wedge \bar{\beta}+\frac{1}{2}\left(\mathrm{i} \phi_{\ell+1}+\varrho_{\ell} \phi_{\ell+1}-\phi_{\ell+1} \varrho_{\ell+1}\right) e^{3} \wedge \bar{\beta}, \\
\mathcal{F}^{\ell+1 \ell} & =-\left(\mathcal{F}^{\ell \ell+1}\right)^{\dagger}=-\frac{1}{2}\left(D \phi_{\ell+1}\right)^{\dagger} \wedge \beta-\frac{1}{2}\left(\mathrm{i} \phi_{\ell+1}^{\dagger}+\phi_{\ell+1}^{\dagger} \varrho_{\ell}-\varrho_{\ell+1} \phi_{\ell+1}^{\dagger}\right) e^{3} \wedge \beta, \\
\mathcal{F}^{k 0} & =\frac{1}{2} D \phi_{k+1} \wedge \bar{\beta}+\frac{1}{2}\left(\mathrm{i} \phi_{k+1}+\varrho_{k} \phi_{k+1}-\phi_{k+1} \varrho_{0}\right) e^{3} \wedge \bar{\beta} \\
\mathcal{F}^{0 k} & =-\left(\mathcal{F}^{k 0}\right)^{\dagger}=-\frac{1}{2}\left(D \phi_{k+1}\right)^{\dagger} \wedge \beta-\frac{1}{2}\left(\mathrm{i} \phi_{k+1}^{\dagger}+\phi_{k+1}^{\dagger} \varrho_{k}-\varrho_{0} \phi_{k+1}^{\dagger}\right) e^{3} \wedge \beta
\end{aligned}
$$

Here we defined $F^{\ell}:=\mathrm{d} A^{\ell}+A^{\ell} \wedge A^{\ell}=\frac{1}{2} F_{\hat{\mu} \hat{\nu}}^{\ell}(x) \mathrm{d} x^{\hat{\mu}} \wedge \mathrm{d} x^{\hat{\nu}}$ and introduced the bifundamental covariant derivatives

$$
D \phi_{\ell+1}:=\mathrm{d} \phi_{\ell+1}+A^{\ell} \phi_{\ell+1}-\phi_{\ell+1} A^{\ell+1},
$$


with $A^{k+1}:=A^{0}, \varrho_{k+1}:=\varrho_{0}$ and $\ell=0,1, \ldots, k$.

The quiver gauge theory action functional (4.9) is obtained by substituting in (4.18)-(4.23) to get

$$
\begin{aligned}
S_{\mathrm{YM}}=\frac{\pi r^{3}}{6(k+1)} \int_{M} \mathrm{~d} \operatorname{vol}_{M} & \sum_{\ell=0}^{k} \operatorname{tr}_{N_{\ell}}\left(F_{\hat{\mu} \hat{\nu}}^{\ell} F^{\ell \hat{\mu} \hat{\nu}}+\frac{1}{r^{2}}\left(D_{\hat{\mu}} \phi_{\ell+1}\right)\left(D^{\hat{\mu}} \phi_{\ell+1}\right)^{\dagger}\right. \\
& +\frac{1}{r^{2}}\left(D_{\hat{\mu}} \phi_{\ell}\right)^{\dagger}\left(D^{\hat{\mu}} \phi_{\ell}\right)+\frac{1}{2 r^{4}}\left(2 \mathrm{i} \varrho_{\ell}+\phi_{\ell}^{\dagger} \phi_{\ell}-\phi_{\ell+1} \phi_{\ell+1}^{\dagger}\right)^{2} \\
& +\frac{1}{r^{4}}\left(\mathrm{i} \phi_{\ell+1}+\varrho_{\ell} \phi_{\ell+1}-\phi_{\ell+1} \varrho_{\ell+1}\right)\left(\mathrm{i} \phi_{\ell+1}+\varrho_{\ell} \phi_{\ell+1}-\phi_{\ell+1} \varrho_{\ell+1}\right)^{\dagger} \\
& \left.+\frac{1}{r^{4}}\left(\mathrm{i} \phi_{\ell}+\varrho_{\ell-1} \phi_{\ell}-\phi_{\ell} \varrho_{\ell}\right)^{\dagger}\left(\mathrm{i} \phi_{\ell}+\varrho_{\ell-1} \phi_{\ell}-\phi_{\ell} \varrho_{\ell}\right)\right) .
\end{aligned}
$$

The corresponding F-term relations are

$$
\phi_{\ell+1} \varrho_{\ell+1}=\varrho_{\ell} \phi_{\ell+1}+\mathrm{i} \phi_{\ell+1}
$$

for $\ell=0,1, \ldots, k$, which give the relations $\mathrm{R}_{k}$ for the quiver $\mathrm{Q}_{\widehat{A}_{k}}$. The $\mathrm{D}$-term constraints are given by

$$
\phi_{\ell+1} \phi_{\ell+1}^{\dagger}-\phi_{\ell}^{\dagger} \phi_{\ell}=2 \mathrm{i} \varrho_{\ell}
$$

for $\ell=0,1, \ldots, k$.

Reduction to $\boldsymbol{A}_{\boldsymbol{k}+1}$ quiver gauge theory. We will now compare the $\widehat{A}_{k}$-type quiver gauge theory for $k=2 q$ with that based on the $A_{k+1}$ quiver which arises from SU(2)-equivariant dimensional reduction over $\mathbb{C} P^{1}[22,9]$. For this, we notice that for $\varphi=0$ in (2.34) the field $B$ becomes an $\mathfrak{s u}(2)$-valued one-form on $\mathbb{C} P^{1} \hookrightarrow S^{3} / \mathbb{Z}_{k+1}$ with $e^{3}=-\mathrm{i} a_{1}$, i.e. fixing $\varphi=0$ reduces our geometry to the base $\mathbb{C} P^{1}$ of the fibration (2.10). The same effect can also be achieved by taking the limit $k \rightarrow \infty$ as was discussed in e.g. [23]; however, here we will keep $k$ finite since we want to compare the $\widehat{A}_{k}$-type and $A_{k+1}$ quiver gauge theories.

Let us now describe the dynamical transition from the cyclic $\widehat{A}_{k}$-type quiver (3.25) to the linear $A_{k+1}$ quiver

$$
\bullet \longrightarrow \bullet \longrightarrow \longrightarrow
$$

which arises by restricting an irreducible representation of $\mathrm{SU}(2)$ on $\mathbb{C}^{k+1}$ to the subgroup $\mathrm{U}(1) \subset$ $\mathrm{SU}(2)$ [9]. In terms of the matrices (3.23) and (3.24) it can be realized by putting

$$
\phi_{k+1}=0
$$

and fixing

$$
\varrho_{\ell}=-\frac{\mathrm{i}}{2}(k-2 \ell) \mathbf{1}_{\mathbb{C}^{N_{\ell}}} \quad \text { for } \quad \ell=0,1, \ldots, k .
$$

For this choice the relations (4.26) are automatically satisfied, and the expressions (4.19)-(4.25) reduce to the expressions for the $A_{k+1}$ quiver gauge theory derived in [9].

Note that the case $k=2 q+1$ reduces in this limit to an $A_{q+1} \sqcup A_{q+1}$ quiver gauge theory, which can be obtained within the framework of [9] by restricting instead a reducible representation of $\mathrm{SU}(2)$ on $\mathbb{C}^{q+1} \oplus \mathbb{C}^{q+1}$.

Reductions to $\boldsymbol{D}_{\boldsymbol{k}}$ and $\boldsymbol{E}_{\boldsymbol{k}}$ quiver gauge theories. For a general ADE group quotient $\Gamma \rightarrow \Gamma_{0}$, setting $\varphi=0$ in $(2.14)$ again reduces the geometry to the base $\mathbb{C} P^{1} / \Gamma_{0}$ of the Seifert fibration (2.6). Similar reductions as in the $\widehat{A}_{k}$ case above then define a quiver gauge theory 
on $M$ based on the ordinary (unextended) Dynkin diagram of ADE type, which arises from a putative equivalence between $\mathrm{SU}(2)$-equivariant vector bundles on $M \times \mathbb{C} P^{1} / \Gamma_{0}$ and $\Gamma_{0} \times \mathrm{U}(1)$ equivariant vector bundles on $M$, where $\mathrm{SU}(2)$ acts trivially on the manifold $M$; this V-bundle equivalence generalizes the equivalences of $[22,9]$ to the equivariant dimensional reduction over two-dimensional orbifolds $\mathbb{C} P^{1} / \Gamma_{0}$.

\section{Instantons on orbifolds and quiver varieties}

McKay quivers. We begin by describing a class of quivers of paramount importance to the study of instantons on the Calabi-Yau cones $C\left(S^{3} / \Gamma\right)$ and their relevance to the McKay correspondence; see e.g. $[24,25]$ for further details. Given the representation $V_{R}$ of the orbifold group $\Gamma$ from (3.2), consider the decomposition

$$
V_{R} \otimes V_{\ell}=\bigoplus_{\ell^{\prime}=0}^{r_{\Gamma}} a_{\ell \ell^{\prime}} V_{\ell^{\prime}}
$$

with tensor product multiplicities $a_{\ell \ell^{\prime}}=\operatorname{dim}_{\mathbb{C}} \operatorname{Hom}_{\Gamma}\left(V_{\ell}, V_{R} \otimes V_{\ell^{\prime}}\right) \in \mathbb{Z}_{\geq 0}$. The McKay quiver $Q_{\Gamma, R}$ has vertices labelled by the irreducible representations of the orbifold group $\Gamma$, i.e. the vertices of the associated extended ADE Dynkin diagram, and $a_{\ell \ell^{\prime}}$ arrows from vertex $\ell$ to vertex $\ell^{\prime}$. In general, the matrix $A=\left(a_{\ell \ell^{\prime}}\right)$ is not symmetric unless $V_{R}$ is a self-dual representation of $\Gamma$; in that case $\mathrm{Q}_{\Gamma, R}$ is the double $\overline{\mathrm{Q}}$ of some quiver $\mathrm{Q}$, i.e. the quiver with the same set of nodes $\overline{\mathrm{Q}}_{0}=\mathrm{Q}_{0}$ and with arrow set $\bar{Q}_{1}=Q_{1} \sqcup Q_{1}^{\text {op }}$, where $Q^{\text {op }}$ is the opposite quiver obtained from $\mathrm{Q}$ by reversing the orientation of the edges. The quiver $Q_{\Gamma, R}$ contains no loop edges if and only if the trivial representation $V_{0}$ does not appear in the decomposition (3.2) of $V_{R}$ into irreducible $\Gamma$-modules, i.e. $N_{0}=0$.

The McKay correspondence is the observation that for the self-dual fundamental representation $V_{R}=\mathbb{C}^{2}$ of $\Gamma \subset \mathrm{SU}(2)$, the matrix $A=A_{\Gamma}$ is the adjacency matrix of the simply-laced extended Dynkin diagram corresponding to $\Gamma$; hence the McKay quiver $Q_{\Gamma, \mathbb{C}^{2}}$ associated to the fundamental representation is the double quiver of the affine ADE Dynkin graph with any choice of orientation. A simple application of Schur's lemma shows that the representations of $Q_{\Gamma, \mathbb{C}^{2}}$ into $R$ correspond bijectively to $\Gamma$-equivariant homomorphisms $V_{R} \rightarrow \mathbb{C}^{2} \otimes V_{R}$, since by (3.2) and (5.1) one has

$$
\operatorname{Hom}_{\Gamma}\left(V_{R}, \mathbb{C}^{2} \otimes V_{R}\right)=\bigoplus_{\ell, \ell^{\prime}=0}^{r_{\Gamma}} a_{\ell \ell^{\prime}} \operatorname{Hom}_{\mathbb{C}}\left(R_{\ell}, R_{\ell^{\prime}}\right),
$$

and so given a morphism in $\operatorname{Hom}_{\Gamma}\left(V_{R}, \mathbb{C}^{2} \otimes V_{R}\right)$ one can pair it with an arrow from $\ell$ to $\ell^{\prime}$ to get a map $R_{\ell} \rightarrow R_{\ell^{\prime}}$; hence we have

$$
\operatorname{Hom}_{\Gamma}\left(V_{R}, \mathbb{C}^{2} \otimes V_{R}\right)=\operatorname{Rep}_{\mathrm{Q}_{\Gamma, \mathbb{C}^{2}}}(R)
$$

The McKay quiver also comes equiped with a set of relations $\mathrm{R}_{\Gamma, \mathbb{C}^{2}}$ that are determined by mapping $B \in \operatorname{Rep}_{Q_{\Gamma, \mathbb{C}^{2}}}(R) \cong \mathfrak{u}(N)$ to the corresponding matrices $B_{1}, B_{2} \in \operatorname{End}_{\mathbb{C}}\left(V_{R}\right)$ under (5.3) with respect to the canonical basis of $\mathbb{C}^{2}$, which obey $\Gamma$-equivariance conditions derived from (2.20) and (3.3) as

$$
\gamma(g) B_{1} \gamma(g)^{-1}=\left(g^{-1}\right)_{1}{ }^{\alpha} B_{\alpha} \quad \text { and } \quad \gamma(g) B_{2} \gamma(g)^{-1}=\left(g^{-1}\right)_{2}{ }^{\alpha} B_{\alpha}
$$

for all $g \in \Gamma$. Then the relations $\mathrm{R}_{\Gamma, \mathbb{C}^{2}}$ for $\mathrm{Q}_{\Gamma, \mathbb{C}^{2}}$ are given by the commutation relations

$$
\left[B_{1}, B_{2}\right]=0 \text {. }
$$

Since $\Gamma \subset \mathrm{SU}(2)$, the commutator $\left[B_{1}, B_{2}\right]$ is $\Gamma$-invariant and hence is valued in the Lie algebra

$$
\mathfrak{g}(R):=\operatorname{End}_{\Gamma}^{a}\left(V_{R}\right)=\bigoplus_{\ell=0}^{r_{\Gamma}} \mathfrak{u}\left(N_{\ell}\right)
$$


of the broken gauge group (3.9).

Generalized instanton equations. Introduce closed two-forms on $\mathbb{R}^{4}$ given by

$$
\omega^{a}:=\frac{1}{2} \eta_{\mu \nu}^{a} \mathrm{~d} y^{\mu} \wedge \mathrm{d} y^{\nu}
$$

where $\eta_{\mu \nu}^{a}$ are components of the self-dual 't Hooft tensor given by

$$
\eta_{b c}^{a}=\varepsilon_{b c}^{a} \quad \text { and } \quad \eta_{b 4}^{a}=-\eta_{4 b}^{a}=\delta_{b}^{a} .
$$

The two-forms $\omega^{a}, a=1,2,3$, are self-dual,

$$
* \omega^{a}=\omega^{a},
$$

where $*$ is the Hodge duality operator for the standard flat Euclidean metric on $\mathbb{R}^{4}$. They define a hyper-Kähler structure on $\mathbb{R}^{4}$ with complex structures

$$
\left(J^{a}\right)_{\nu}^{\mu}=\omega_{\nu \lambda}^{a} \delta^{\lambda \mu}
$$

where the complex structure $J^{3}$ identifies $\mathbb{R}^{4}=\mathbb{C}^{2}$ with the complex coordinates $(2.17)$.

Let $\underline{A}=W_{\mu} \mathrm{d} y^{\mu}$ be a connection on the (trivial) V-bundle $\underline{V_{R}}:=\mathbb{R}^{4} \times V_{R}$ of rank $N$ over $\mathbb{R}^{4} / \Gamma$ with curvature $\underline{F}=\mathrm{d} \underline{A}+\underline{A} \wedge \underline{A}$. In the following we shall study the moduli space of solutions (with finite topological charge) to the generalized instanton equations [18]

$$
* \underline{F}+\underline{F}=2 \omega^{a} \Xi_{a},
$$

where

$$
\Xi_{a}:=\operatorname{diag}\left(\mathrm{i} \xi_{0}^{a} \mathbf{1}_{N_{0}}, \mathrm{i} \xi_{1}^{a} \mathbf{1}_{N_{1}}, \ldots, \mathrm{i} \xi_{r_{\Gamma}}^{a} \mathbf{1}_{N_{r_{\Gamma}}}\right)
$$

for $a=1,2,3$ are elements of the center of the Lie algebra (5.6) and the Fayet-Iliopoulos parameter $\xi=\left(\xi_{\ell}^{a}\right)$ is the linearization of the bundle action. For $\xi=0$ the equations (5.11) are the antiself-dual Yang-Mills equations on the orbifold $\mathbb{R}^{4} / \Gamma$, while for $\xi^{1}=\xi^{2}=0, \xi^{3} \neq 0$ they become the Hermitian Yang-Mills equations which imply that the V-bundle $V_{R}$ is a (semi-)stable vector bundle $[26,27]$; for generic $\xi \neq 0$ they correspond to BPS-type equations for Yang-Mills theory with sources [18].

Moduli spaces of translationally-invariant instantons. We will provide a geometric interpretation of the McKay quiver in terms of moduli spaces of translationally-invariant $\Gamma$-equivariant instantons on the V-bundle $\underline{V_{R}}$. Dimensional reduction of the equations (5.11) leads to the matrix equations

$$
\begin{aligned}
& {\left[W_{2}, W_{3}\right]+\left[W_{1}, W_{4}\right]=\Xi_{1},} \\
& {\left[W_{3}, W_{1}\right]+\left[W_{2}, W_{4}\right]=\Xi_{2},} \\
& {\left[W_{1}, W_{2}\right]+\left[W_{3}, W_{4}\right]=\Xi_{3},}
\end{aligned}
$$

where the constant matrices $W_{\mu}$ with $\mu=1,2,3,4$ take values in the Lie algebra $\mathfrak{u}(N)$ and can be regarded as components of the gauge potential along the internal space of the dimensional reduction. The reduced equations (5.13) can be interpreted as hyper-Kähler moment map equations, and hence the moduli space of translationally-invariant instantons is given by a hyper-Kähler quotient [18].

When $R=\widehat{V}$ is the multiplicity space (3.1) of the self-dual regular representation of $\Gamma$ (so that $N=|\Gamma|$ ), this finite-dimensional hyper-Kähler quotient construction was used by Kronheimer in [12] to construct a family of four-dimensional hyper-Kähler manifolds $M_{\xi}$. The representation theory of the orbifold group $\Gamma$ and the McKay correspondence are encoded in the property that 
$M_{\xi}$ for generic $\xi \neq 0$ is an ALE gravitational instanton, i.e. it is diffeomorphic to the minimal smooth resolution of the Kleinian singularity $M_{0}=\mathbb{C}^{2} / \Gamma$. The birational morphisms $\pi: M_{\xi} \rightarrow M_{0}$ are isomorphisms over the cone $C\left(S^{3} / \Gamma\right)$ whose exceptional fibre $\pi^{-1}(0)$ is a graph of rational curves $\Sigma_{\ell} \cong \mathbb{C} P^{1}, \ell=1, \ldots, r_{\Gamma}$ which is dual to the ordinary (unextended) ADE Dynkin graph associated to $\Gamma$; the parameters $\xi=\left(\xi_{\ell}^{a}\right)$ are the periods of the trisymplectic structure over $\Sigma_{\ell}$ under the isomorphism $\mathrm{H}^{2}\left(M_{\xi} ; \mathbb{R}\right) \cong \mathbb{R}^{r_{\Gamma}+1}[12]$. The variety $M_{\xi}$ also inherits a natural hyperKähler metric over $C\left(S^{3} / \Gamma\right)$ parameterized by $\xi$ which is asymptotically locally Euclidean (ALE), i.e. it approximates the flat Euclidean metric on the orbifold $\mathbb{R}^{4} / \Gamma$ at the end of $M_{\xi}$ up to order $r^{-4}$.

One way to regard the complex deformation $M_{\xi}$ is by modifying the polynomial equation (2.23) in $\mathbb{C}^{3}$ which deforms the cone $C\left(S^{3} / \Gamma\right)$ to

$$
f_{\Gamma}(x, y, z ; \vec{t})=0
$$

where $\vec{t}=\left(t_{0}, t_{1}, \ldots, t_{r_{\Gamma}}\right)$ are coordinates on the base of the deformation related to the periods $\xi_{\ell}^{a}$; see e.g. [7, Sect. 2.1] for details of this construction. Explicitly, for the five classes of Sasaki-Einstein three-manifolds we have

$$
\begin{aligned}
f_{\mathbb{Z}_{k+1}}(x, y, z ; \vec{t}) & =P_{k+1}(x ; \vec{t})+y^{2}+z^{2}, \\
f_{\mathbb{D}_{k-2}^{*}}(x, y, z ; \vec{t}) & =x^{k-1}+Q_{k-2}(x ; \vec{t})+t_{0} y+x y^{2}+z^{2}, \\
f_{\mathbb{T}^{*}}(x, y, z ; \vec{t}) & =y^{3}+Q_{2}(x ; \vec{t})+P_{4}(x ; \vec{t})+z^{2}, \\
f_{\mathbb{O}^{*}}(x, y, z ; \vec{t}) & =y^{3}+P_{3}(x ; \vec{t})+Q_{4}(x ; \vec{t})+z^{2}, \\
f_{\mathbb{I}^{*}}(x, y, z ; \vec{t}) & =y^{3}+Q_{3}(x ; \vec{t})+P_{5}(x ; \vec{t})+z^{2},
\end{aligned}
$$

where $P_{k}(x ; \vec{t})=x^{k}+\sum_{\ell=0}^{k} t_{\ell} x^{k-\ell}$ and $Q_{k}(x ; \vec{t})=\sum_{\ell=1}^{k+1} t_{\ell} x^{k-\ell+1}$. This realizes $M_{\xi}$ as a fibration over the $x$-plane with generic fibre $\mathbb{C}^{*}$ (for $\mathrm{A}$ and $\mathrm{D}$ series) or elliptic curves (for E series).

Nakajima quiver varieties. Kronheimer's construction can be interpreted in terms of moduli spaces of representations of the McKay quiver with relations $\left(Q_{\Gamma, \mathbb{C}^{2}}, \mathrm{R}_{\Gamma, \mathbb{C}^{2}}\right)$ into the regular representation space (3.1) of $\Gamma$; see [28] for further details. This moduli space is a particular example of a Nakajima quiver variety [29].

For a quiver $\mathrm{Q}$ based on $\Gamma$ and a $\mathrm{Q}_{0}$-graded vector space $R$, introduce stability parameters $\xi: Q_{0} \rightarrow \mathbb{R}^{3} \cong \mathbb{C} \oplus \mathbb{R}$ and identify the Lie algebra $\mathfrak{g}(R)$ with its dual $\mathfrak{g}(R)^{*}$ using the Cartan-Killing form. The vector space $\operatorname{Rep}_{\bar{Q}}(R)$ carries a metric defined by the Hilbert-Schmidt norm

$$
\|B\|^{2}:=\sum_{e \in \overline{\mathrm{Q}}_{1}} \operatorname{tr}_{N_{\mathrm{s}(e)}} B_{e} B_{e}^{\dagger}
$$

and a holomorphic symplectic form

$$
\omega_{\mathbb{C}}\left(B, B^{\prime}\right):=\sum_{e \in \overline{\mathbf{Q}}_{1}} \epsilon(e) \operatorname{tr}_{N_{\mathrm{s}(e)}} B_{e} B_{\bar{e}}^{\prime}
$$

where $\bar{e} \in \mathrm{Q}_{1}^{\mathrm{op}}$ is the reverse edge of $e$ with $\epsilon(e)=1$ and $\epsilon(\bar{e})=-1$ for $e \in \mathrm{Q}_{1}$. It decomposes as a sum of Lagrangian subspaces $\operatorname{Rep}_{\bar{Q}}(R)=\operatorname{Rep}_{\mathbf{Q}}(R) \oplus \operatorname{Rep}_{Q_{\text {op }}}(R)$ with $\operatorname{Rep}_{\mathrm{Q}_{\mathrm{op}}}(R) \cong \operatorname{Rep}_{\mathrm{Q}}(R)^{*}$, which geometrically identifies it as the cotangent bundle $\operatorname{Rep}_{\bar{Q}}(R) \cong T^{*} \mathcal{R e p}_{\mathrm{Q}}(R)$; hence it is naturally a quaternionic vector space which gives it the structure of a flat hyper-Kähler manifold that is preserved by the bifundamental action (3.14) of the gauge group (3.9). The corresponding $(1,1)$ form is

$$
\omega_{\mathbb{R}}\left(B, B^{\prime}\right):=\frac{1}{2} \sum_{e \in \overline{\mathrm{Q}}_{1}} \epsilon(e) \operatorname{tr}_{N_{\mathrm{s}(e)}}\left(B_{e} B_{e}^{\prime \dagger}-B_{\bar{e}}^{\dagger} B_{\bar{e}}^{\prime}\right) .
$$


Then the quiver variety associated to $Q$ and $R$ is the hyper-Kähler quotient

$$
\mathcal{X}_{\xi}(\mathrm{Q}, R):=\operatorname{Rep}_{\overline{\mathrm{Q}}}(R) / / \xi_{\xi} \mathrm{PG}(R)
$$

by the corresponding hyper-Kähler moment map $\mu=\left(\mu_{\mathbb{C}}, \mu_{\mathbb{R}}\right): \mathcal{R e p}_{\overline{\mathrm{Q}}}(R) \rightarrow \mathbb{R}^{3} \otimes \mathfrak{g}(R)$ vanishing at the origin, where $\mu_{\mathbb{C}}: \operatorname{Rep}_{\overline{\mathbf{Q}}}(R) \rightarrow \mathfrak{g}(R) \otimes \mathbb{C}$ is defined by its components

$$
\mu_{\mathbb{C}}(B)_{\ell}=\sum_{e \in \mathbf{s}^{-1}(\ell)} \epsilon(e) B_{e} B_{\bar{e}}
$$

while $\mu_{\mathbb{R}}: \operatorname{Rep}_{\bar{Q}}(R) \rightarrow \mathfrak{g}(R)$ is defined by

$$
\mu_{\mathbb{R}}(B)_{\ell}=\frac{\mathrm{i}}{2} \sum_{e \in \mathrm{s}^{-1}(\ell)}\left(B_{e} B_{e}^{\dagger}-B_{\bar{e}}^{\dagger} B_{\bar{e}}\right)
$$

for $\ell=0,1, \ldots, r_{\Gamma}$. There are relations

$$
\sum_{\ell=0}^{r_{\Gamma}} \operatorname{tr}_{N_{\ell}} \mu_{\mathbb{C}}(B)_{\ell}=0=\sum_{\ell=0}^{r_{\Gamma}} \operatorname{tr}_{N_{\ell}} \mu_{\mathbb{R}}(B)_{\ell}
$$

which follow from cyclicity of the traces.

The quiver variety is then constructed via suitable quotients of the level set $\mu^{-1}(\Xi)$; this necessitates the traceless condition

$$
\sum_{\ell=0}^{r_{\Gamma}} \xi_{\ell}^{a} N_{\ell}=0 \quad \text { for } \quad a=1,2,3 .
$$

The canonical map $\mathcal{X}_{\xi}(\mathrm{Q}, R) \rightarrow \mathcal{X}_{0}(\mathrm{Q}, R)$ is a smooth (hyper-Kähler) resolution of singularities for generic values of $\xi$. By choosing $\xi$ such that the gauge group $\mathrm{PG}(R)$ acts freely, the dimension of the hyper-Kähler quotient (and of the vacuum moduli space of the corresponding quiver gauge theory) is given by [30, Sect. 3.3]

$$
\operatorname{dim}_{\mathbb{R}} \mathcal{X}_{\xi}(\mathrm{Q}, R)=\operatorname{dim}_{\mathbb{R}} \operatorname{Rep}_{\overline{\mathrm{Q}}}(R)-4 \operatorname{dim}_{\mathbb{R}} \mathrm{PG}(R)=4-2 \vec{N} \cdot C_{\mathrm{Q}} \vec{N}
$$

where the generalized Cartan matrix $C_{\mathrm{Q}}$ of the quiver $\mathrm{Q}$ is defined by

$$
\vec{N} \cdot C_{\mathrm{Q}} \vec{N}^{\prime}=2 \sum_{\ell=0}^{r_{\Gamma}} N_{\ell} N_{\ell}^{\prime}-\sum_{e \in \overline{\mathrm{Q}}_{1}} N_{\mathrm{s}(e)} N_{\mathrm{t}(e)}^{\prime}
$$

for dimension vectors $\vec{N}:=\left(N_{0}, N_{1}, \ldots, N_{r_{\Gamma}}\right)$ and $\vec{N}^{\prime}:=\left(N_{0}^{\prime}, N_{1}^{\prime}, \ldots, N_{r_{\Gamma}}^{\prime}\right)$ of two quiver representations $R$ and $R^{\prime}$; note that $\vec{N} \cdot C_{\mathrm{Q}} \vec{N} \in 2 \mathbb{Z}$. It follows that if $\vec{N} \cdot C_{\mathrm{Q}} \vec{N}>2$ then the representation $R$ is decomposable, while if $\vec{N} \cdot C_{\mathrm{Q}} \vec{N}=2$ the representation is rigid, i.e. it has no moduli. Dimension vectors $\vec{N}$ of indecomposable quiver representations are called roots of the quiver (see [31, Sect. 2]); in particular rigid representations correspond to real roots. Imaginary roots have Cartan form with $\vec{N} \cdot C_{\mathrm{Q}} \vec{N} \leq 0$ and parameterize moduli spaces of $\operatorname{dimension} \operatorname{dim}_{\mathbb{R}} \mathcal{X}_{\xi}(\mathrm{Q}, R) \geq 4$.

McKay quiver varieties. The relations (5.5) for the McKay quiver $\bar{Q}=Q_{\Gamma, \mathbb{C}^{2}}$ can be written using the isomorphism (5.3) as $\mu_{\mathbb{C}}(B)=0$; in fact, one may set $\Xi_{1}=\Xi_{2}=0$ by a non-analytic change of coordinates on the representation space (3.13) $[12,32]$. On the other hand, the real moment map equations $\mu_{\mathbb{R}}(B)=\Xi_{3}$ in this case can be written as

$$
\left[B_{1}, B_{1}^{\dagger}\right]+\left[B_{2}, B_{2}^{\dagger}\right]=\frac{\mathrm{i}}{2} \Xi_{3} .
$$


In this instance the generalized Cartan matrix $C_{\mathrm{Q}}$ coincides with the extended Cartan matrix $\widehat{C}_{\Gamma}=2 \mathbf{1}_{\mathbb{C}^{r}+1}-A_{\Gamma}$ of the simply-laced affine Lie algebra $\widehat{\mathfrak{g}}_{\Gamma}$ associated to $\Gamma$.

The Kronheimer construction is then obtained by specialising to the quiver variety associated to the McKay quiver with relations $\left(\mathrm{Q}_{\Gamma, \mathbb{C}^{2}}, \mathrm{R}_{\Gamma, \mathbb{C}^{2}}\right)$ and the multiplicity space $R=\widehat{V}$, so that

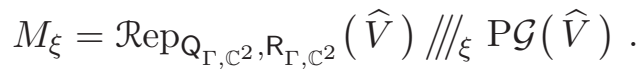

Explicitly, the equations (5.13) and $\mu(B)=\Xi$ are related by identifying the $\Gamma$-equivariant matrices $B_{1}:=\frac{1}{2}\left(-W_{4}+\mathrm{i} W_{3}\right)$ and $B_{2}:=\frac{1}{2}\left(W_{1}+\mathrm{i} W_{2}\right)$ with $B \in \mathcal{R e p}_{Q_{\Gamma, \mathbb{C}^{2}}}(\widehat{V})$ under the isomorphism (5.3). Since $\Gamma$ acts freely on the cone $C\left(S^{3}\right)=\mathbb{C}^{2} \backslash\{0\}$, the variety (5.27) is coordinatized by a fixed simultaneous eigenvalue pair $\left(z_{1}, z_{2}\right)$ of the commuting matrices $B_{1}, B_{2}$ modulo its $\Gamma$-orbit, where the orbit is the regular representation on the coordinates. Since the dimension vector $\vec{n}=$ $\left(n_{0}, n_{1}, \ldots, n_{r_{\Gamma}}\right)$ spans the kernel of the extended Cartan matrix $\widehat{C}_{\Gamma}$, it follows from the general dimension formula (5.24) that the quiver variety (5.27) has dimension $\operatorname{dim}_{\mathbb{R}} M_{\xi}=4$.

Superconformal quiver gauge theory. The construction of quiver varieties for $R=\widehat{V}$ has a natural interpretation in the four-dimensional $\mathcal{N}=2$ superconformal quiver gauge theory on the worldvolume of $n \mathrm{D} p$-branes probing a single $\mathrm{D}(p+4)$-brane placed at the orbifold singularity of $\mathbb{C}^{2} / \Gamma[4,5,7]$. The field theory has gauge group (3.9), with $N_{\ell}=n n_{\ell}$ the number of constituent fractional $\mathrm{D} p$-branes, and is based on the McKay quiver $\mathrm{Q}_{\Gamma, \mathbb{C}^{2}}$ : At each vertex $\ell=0,1, \ldots, r_{\Gamma}$ there is an $\mathcal{N}=2$ vector multiplet which corresponds to an $\mathcal{N}=1$ chiral multiplet $\varphi_{\ell}$ transforming in the adjoint representation of $\mathrm{U}\left(N_{\ell}\right)$, while for each edge $e$ there is a bifundamental hypermultiplet which corresponds to a pair of $\mathcal{N}=1$ chiral multiplets $\left(B_{e}, B_{\bar{e}}\right)$ with $B_{e}$ a complex matrix transforming as in (3.14). The cubic $\mathcal{N}=1$ superpotential is then determined from the complex moment map given by (5.20) as

$$
W_{\Gamma}(B, \varphi)=\sum_{\ell=0}^{r_{\Gamma}} \operatorname{tr}_{N_{\ell}} \mu_{\mathbb{C}}(B)_{\ell} \varphi_{\ell} .
$$

One can modify the superpotential by shifting it with complex Fayet-Iliopoulos terms to

$$
W_{\Gamma}(B, \varphi)_{\xi}=W_{\Gamma}(B, \varphi)-\sum_{\ell=0}^{r_{\Gamma}} \mathrm{i} \xi_{\ell}^{\mathbb{C}} \operatorname{tr}_{N_{\ell}} \varphi_{\ell}
$$

whilst still preserving $\mathcal{N}=2$ supersymmetry. In the supersymmetric vacuum state all fermion fields are set to zero, with the scalar fields taking constant vacuum expectation values. The F-term equations

$$
\frac{\partial W_{\Gamma}(B, \varphi)_{\xi}}{\partial \varphi_{\ell}}=0
$$

then reproduce the deformed McKay quiver relations $\mu_{\mathbb{C}}(B)_{\ell}=\mathrm{i} \xi_{\ell}^{\mathbb{C}}$ for $\ell=0,1, \ldots, r_{\Gamma}$. The Dterm equations instead encode qualitative stability information about the (non-degenerate) kinetic term given by the metric (5.16), and they correspond to the level sets (5.26) of the real moment map from (5.21). By factoring solutions to these equations by the action of the gauge group (3.9) we obtain the vacuum moduli space. It follows that the quiver variety $\mathcal{X}_{\xi}(\mathrm{Q}, R)$ for $\bar{Q}=\mathrm{Q}_{\Gamma, \mathbb{C}^{2}}$ and $R=\widehat{V}$ can be realized as the Higgs branch of this quiver gauge theory.

Moduli spaces of framed instantons. We can work instead with framed McKay quivers [14, $29,25]$ which are obtained by adding a node with doubled arrows to each vertex of the McKay quiver with relations $\left(Q_{\Gamma, \mathbb{C}^{2}}, R_{\Gamma, \mathbb{C}^{2}}\right)$, where in general the extra nodes correspond to $\Gamma$-modules 
$V_{S}$ which parameterize the holonomies of instanton gauge connections at infinity. The extended representation space is given by

$$
\operatorname{Rep}_{\bar{Q}}(R, S):=\operatorname{Rep}_{\overline{\mathbf{Q}}}(R) \oplus \bigoplus_{\ell=0}^{r_{\Gamma}} \operatorname{Hom}_{\mathbb{C}}\left(R_{\ell}, S_{\ell}\right) \oplus \operatorname{Hom}_{\mathbb{C}}\left(S_{\ell}, R_{\ell}\right) .
$$

The corresponding quiver varieties $\mathcal{X}_{\xi}(\mathrm{Q} ; R, S)$ parameterize moduli of framed instantons on the ALE spaces $M_{\xi}$, or alternatively the Higgs branches of quiver gauge theories of $N>1 \mathrm{D}(p+4)$ branes at the orbifold singularity with framing corresponding to the addition of probe $\mathrm{D} p$-branes to the $\mathrm{D}(p+4)$-branes [4]; in this case the moduli arise as ADHM data. A connection on a framed vector bundle $E \rightarrow M_{\xi}$ of rank $N$ is specified by the Chern classes of $E$ and a flat connection at the end of $M_{\xi}$, which is isomorphic to the Sasaki-Einstein space $S^{3} / \Gamma$. The McKay correspondence can then be stated as a one-to-one correspondence between flat $\mathrm{U}(N)$ connections on $S^{3} / \Gamma$, which correspond to representations $V_{S}$ of the fundamental group $\pi_{1}\left(S^{3} / \Gamma\right)=\Gamma$ in $\mathrm{U}(N)$, and integrable highest weight representations of the affine Lie algebra $\widehat{\mathfrak{g}}_{\Gamma}$ of ADE type associated to $\Gamma$ at level $N$. In [29] Nakajima constructs natural representations of $\widehat{\mathfrak{g}}_{\Gamma}$ at level $N$ on the cohomology of the quiver varieties in terms of geometric Hecke correspondences.

In general, although the ADHM construction of Yang-Mills instantons on ALE spaces is a finitedimensional hyper-Kähler quotient construction, it cannot be presented as translationally-invariant anti-self-duality equations with Fayet-Iliopoulos terms $\xi$ as in (5.13). However, for the instanton configuration of minimal topological charge $c_{1}(E)=0$ and $c_{2}(E)=(|\Gamma|-1) /|\Gamma|$ corresponding to $N_{\ell}=n_{\ell}$ and a single $\mathrm{D}(p+4)$-brane above, the "outer" framing fields decouple [4, Sect. 8.1] and the relevant quiver is the unframed McKay quiver with relations, i.e. the ADHM equations in this case reduce to (5.13) [14, Ex. 3]. This fact is consistent with the constructions of moduli spaces $\mathcal{X}_{\xi}(\mathrm{Q} ; \widehat{V}, S)$ of minimal charge Yang-Mills instantons on the ALE spaces $M_{\xi}$, described in [33] and [32, Sect. 7], as the four-dimensional non-compact hyper-Kähler manifold $M_{\xi}$ itself. Note that the orbifold group $\Gamma$ acts non-trivially on the ADHM matrices but trivially on the vector bundle $E \rightarrow M_{\xi}$, while the embedding of $\Gamma$ in $\mathrm{U}(N)$ is defined by the asymptotic holonomy of the gauge connection rather than by covariance equations such as (3.10).

$\boldsymbol{A}_{\boldsymbol{k}}$. For the cyclic group $\Gamma=\mathbb{Z}_{k+1}$, the fundamental representation (2.37) is reducible with splitting $\mathbb{C}^{2}=V_{1} \oplus V_{k}$, and the decomposition (5.1) reads as

$$
\mathbb{C}^{2} \otimes V_{\ell}=V_{\ell+1} \oplus V_{\ell-1}
$$

for $\ell=0,1, \ldots, k$. Thus only $a_{\ell, \ell \pm 1}=1$ are non-zero, which gives precisely the adjacency matrix of the $\widehat{A}_{k^{-}}$type Dynkin diagram $(2.35)$. The McKay quiver $\mathrm{Q}_{\Gamma, \mathbb{C}^{2}}$ is the corresponding double quiver

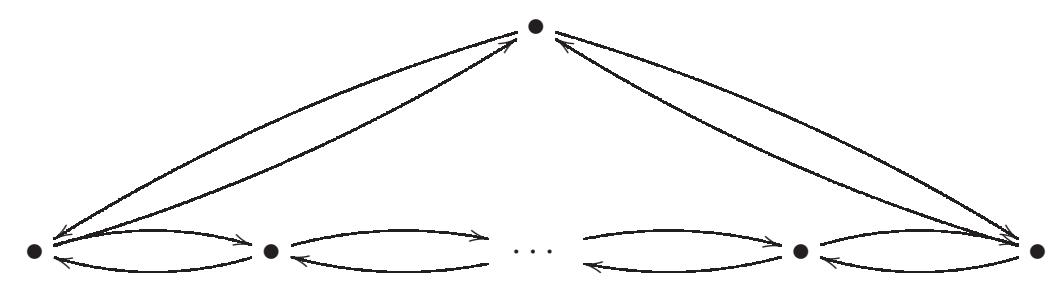

The solutions to the $\Gamma$-equivariance conditions are matrices

$$
B_{1}=\left(\begin{array}{ccccc}
0 & 0 & \cdots & 0 & \psi_{k+1} \\
\psi_{1} & 0 & \ddots & 0 & 0 \\
0 & \psi_{2} & \ddots & \vdots & \vdots \\
\vdots & \ddots & \ddots & 0 & 0 \\
0 & \cdots & 0 & \psi_{k} & 0
\end{array}\right) \quad \text { and } \quad B_{2}=\left(\begin{array}{ccccc}
0 & \varphi_{1} & 0 & \cdots & 0 \\
0 & 0 & \varphi_{2} & \ddots & \vdots \\
\vdots & \vdots & \ddots & \ddots & 0 \\
0 & 0 & \cdots & 0 & \varphi_{k} \\
\varphi_{k+1} & 0 & \cdots & 0 & 0
\end{array}\right)
$$


corresponding respectively to the two summands in (5.32), where the arrows between vertices of the quiver (5.33) are represented by linear maps $\psi_{\ell+1} \in \operatorname{Hom}_{\mathbb{C}}\left(R_{\ell}, R_{\ell+1}\right)$ and $\varphi_{\ell+1} \in \operatorname{Hom}_{\mathbb{C}}\left(R_{\ell+1}, R_{\ell}\right)$ for $\ell=0,1, \ldots, k$. The relations $(5.5)$ read as

$$
\varphi_{\ell+1} \psi_{\ell+1}=\psi_{\ell} \varphi_{\ell}
$$

while the stability conditions (5.26) are given by

$$
\varphi_{\ell+1} \varphi_{\ell+1}^{\dagger}-\varphi_{\ell}^{\dagger} \varphi_{\ell}=\psi_{\ell+1}^{\dagger} \psi_{\ell+1}-\psi_{\ell} \psi_{\ell}^{\dagger}-\frac{1}{2} \xi_{\ell}^{3} \mathbf{1}_{\mathbb{C}^{N_{\ell}}}
$$

on $R_{\ell}$ for each $\ell=0,1, \ldots, k$. By (5.24) the dimension of the corresponding Nakajima quiver variety is given by

$$
\operatorname{dim}_{\mathbb{R}} \mathcal{X}_{\xi}\left(\mathrm{Q}_{\Gamma, \mathbb{C}^{2}}, R\right)=4+4 \sum_{\ell=0}^{k} N_{\ell} N_{\ell+1}-4 \sum_{\ell=0}^{k} N_{\ell}^{2} .
$$

This is equal to four when $N_{\ell}=n$ for all $\ell=0,1, \ldots, k$, and for $n=1$ the quiver variety is a minimal resolution $M_{\xi}$ of the quotient singularity $\mathcal{X}_{0}\left(\mathrm{Q}_{\Gamma, \mathbb{C}^{2}}, \widehat{V}\right)=\mathbb{C}^{2} / \mathbb{Z}_{k+1}$.

\section{Instantons on cones and Nahm equations}

Moduli spaces of spherically symmetric instantons. In this section we describe the moduli spaces of representations of the "Sasakian" quivers from Sect. 3 in an analogous way to those of the McKay quivers from Sect. 5. Comparing the two quiver diagrams (5.33) and (3.25), the two translationally-invariant $\Gamma$-equivariant complex Higgs fields (5.34) yield a quiver based on the Dynkin diagram of type $\widehat{A}_{k}$ involving an arrow between vertices for each Higgs field, while the SU(2)-equivariant Higgs fields (4.12)-(4.13) yield a quiver based on the same Dynkin graph involving an arrow for the complex horizontal Higgs field together with a loop edge for the vertical Higgs field.

The SU(2)-invariant (spherically symmetric) reduction of $\Gamma$-invariant connections on the trivial V-bundle $\underline{V_{R}}$ over $\mathbb{R}^{4} / \Gamma$ is most conveniently done by exploiting conformal invariance of the $\xi$ deformed anti-self-duality equations (5.11) and considering them on the cylinder $\mathbb{R} \times S^{3} / \Gamma$. The connection in these coordinates is written as

$$
\underline{A}=W_{\tau} \mathrm{d} \tau+W_{a} \hat{e}^{a}
$$

where, in contrast to (2.2), the basis of left SU(2)-invariant one-forms

$$
\hat{e}^{a}:=-\frac{1}{r^{2}} \eta_{\mu \nu}^{a} y^{\mu} \mathrm{d} y^{\nu}
$$

on $S^{3}$ are invariant with respect to the action of the orbifold group $\Gamma \subset \mathrm{SU}(2)$; this $\mathrm{SU}(2)$ group is the right-acting factor of the Lorentz group $\mathrm{SO}(4)=\mathrm{SU}(2) \times \mathrm{SU}(2)$ on $\mathbb{R}^{4}$ which preserves the complex structure $J^{3}$ from (5.10), while the left-acting $\mathrm{SU}(2)$ factor does not (only a $\mathrm{U}(1)$ subgroup preserves $J^{3}$ ). Now the matrices $W_{a}$ with $a=1,2,3$ and $W_{\tau}$ depend on the radial coordinate $r=\mathrm{e}^{\tau}$, and by defining

$$
Y_{a}:=\mathrm{e}^{2 \tau} W_{a}, \quad Y_{\tau}:=\mathrm{e}^{2 \tau} W_{\tau} \quad \text { and } \quad s=\mathrm{e}^{-2 \tau}
$$

the equations (5.11) reduce to the ordinary differential equations [18]

$$
2 \frac{\mathrm{d} Y_{a}}{\mathrm{~d} s}=\left[Y_{\tau}, Y_{a}\right]-\frac{1}{2} \varepsilon_{a}^{b c}\left[Y_{b}, Y_{c}\right]+\frac{1}{s^{2}} \Xi_{a}
$$


for the $\Gamma$-invariant functions $Y_{a}, Y_{\tau}: \mathbb{R}_{>0} \rightarrow \mathfrak{g}(R)$ with $a=1,2,3$. For $\Xi_{a}=0$ these equations coincide with the Nahm equations. In contrast to the ADHM-type matrix model, no stability parameters $\Xi$ will be required to define the resolution of singularities of the moduli space of solutions $[15,16,30]$ which will instead arise from the chosen boundary conditions at large $s$; henceforth we set $\Xi=0$ without loss of generality (see also [18, Sect. 5$]$ ).

Let us momentarily consider the case with trivial orbifold group $\Gamma=\{1\}$, and let $\mathcal{G}$ be a compact simple Lie group with maximal torus $\mathcal{T}$; let $\mathcal{G}^{\mathbb{C}}=\mathcal{G} \otimes \mathbb{C}$ and $\mathcal{T}^{\mathbb{C}}=\mathcal{T} \otimes \mathbb{C}$ denote the corresponding complexified groups. Then the equations (6.4) are equivalent to the equations considered by Kronheimer [15, 16] (see also [17]) in the description of SU(2)-invariant instantons [18]. Kronheimer shows in [15] that, for the spherically symmetric Yang-Mills instantons which have minimal topological charge on the cone $\mathbb{R}^{4} \backslash\{0\}=C\left(S^{3}\right)$, under certain boundary conditions the moduli space is again a Calabi-Yau twofold $M_{\xi}^{\prime}$ which is a minimal resolution of $C\left(S^{3} / \Gamma^{\prime}\right)$. This is the moduli space of $\mathrm{SU}(2)$-invariant framed $\mathcal{G}$-instantons on $C\left(S^{3}\right)$ with a "pole" type singularity at the origin, which in terms of Nahm data is characterized by the space of smooth solutions to (6.4) satisfying the boundary conditions

$$
\lim _{s \rightarrow \infty} Y_{\tau}(s)=0, \quad \lim _{s \rightarrow \infty} Y_{a}(s)=T_{a} \quad \text { and } \quad \lim _{s \rightarrow 0} s Y_{a}(s)=I_{a} \quad \text { for } \quad a=1,2,3,
$$

where $T_{a}$ are fixed elements of the Cartan subalgebra $\mathfrak{t}$ of the Lie algebra $\mathfrak{g}$ of $\mathcal{G}$ whose common centralizer is $\mathcal{T}$, and

$$
\left[I_{a}, I_{b}\right]=\varepsilon_{a b}^{c} I_{c}
$$

where the generators $I_{a}$ define an embedding of $\mathrm{SU}(2)$ in $\mathcal{G}$. The elements $T_{a}$ parameterize a hyperKähler structure on the regular coadjoint orbit $\mathcal{G}^{\mathbb{C}} / \mathcal{T}^{\mathbb{C}}$, while the elements $I_{a}$ specify the holonomy of the gauge connection at infinity which generically reduces the orbit to a smaller space. The quotient defining the moduli space is taken by the action of the gauge group $\widehat{\mathcal{G}}$ consisting of gauge transformations $g: \mathbb{R}_{>0} \rightarrow \mathcal{G}$ which are trivial at infinity.

One can solve (6.4) by taking $Y_{a}, Y_{\tau}$ as constant matrices from $\mathfrak{t}$, which produces singular abelian instanton solutions with delta-function sources in the Maxwell equations [15, 16, 18]. By considering $s$-dependent solutions with boundary conditions (6.5), the moduli space can be obtained by an infinite-dimensional hyper-Kähler quotient construction [30, Sect. 5]. For the subregular representation $I=\left(I_{a}\right)$, the resulting manifold is again a four-dimensional hyper-Kähler ALE space $M_{\xi}^{\prime}$ which is a resolution of singularities of the orbifold $M_{0}^{\prime}=\mathbb{C}^{2} / \Gamma^{\prime}$ [15], where the finite subgroup $\Gamma^{\prime} \subset \mathrm{SU}(2)$ is obtained from the homogeneous Dynkin diagram of $\mathcal{G}$; if $\mathcal{G}$ is not simply-laced then this graph is understood as the associated homogeneous Dynkin diagram of ADE-type whose quotient by a finite group of diagram symmetries yields the Dynkin diagram of $\mathcal{G}$, see [34]. The resolution parameters $\xi=\left(\xi_{\ell}^{a}\right)$ for $a=1,2,3$ and $\ell=0,1, \ldots, r_{\Gamma^{\prime}}$ are the periods of the trisymplectic structure determined by $T_{a}$ under the isomorphism $\mathrm{H}^{2}\left(\mathcal{G}^{\mathbb{C}} / \mathcal{T}^{\mathbb{C}} ; \mathbb{R}\right)=\mathrm{H}^{2}(\mathcal{G} / \mathcal{T} ; \mathbb{R}) \cong \mathfrak{t}$; if $\mathcal{G}$ is not of ADE-type then the hyper-Kähler cohomology classes are pullbacks of those associated to $T_{a}$ by the surjective quotient map $\mathfrak{t}^{\prime} \rightarrow \mathfrak{t}$ from the Cartan subalgebra $\mathfrak{t}^{\prime}$ of the associated Lie group with homogeneous Dynkin diagram. For nilpotent orbits with $T_{a}=0$, the moduli spaces of dimension four are cones $C\left(S^{3} / \Gamma^{\prime}\right)$, while in dimensions $\geq 8$ they correspond to the minimal nilpotent orbit, i.e. the orbit of the highest root vector in $\mathfrak{g}$, which is the cone over the 3-Sasakian homogeneous manifold associated to $\mathcal{G}$ [17]. For generic non-regular orbits and smaller $\mathrm{SU}(2)$ representations, if the moduli space is four-dimensional then by [13] it is a disjoint union of ALE spaces and cones.

For a non-trivial orbifold group $\Gamma$, we can repeat this construction with $\mathcal{G}=\mathcal{G}(R)$. Then the moduli space of solutions to the Nahm equations (6.4) with the boundary conditions (6.5) is a product of $r_{\Gamma}+1$ moduli spaces of Nahm data associated with each factor $\mathrm{U}\left(N_{\ell}\right)$ of the gauge group (3.9) for $\ell=0,1, \ldots, r_{\Gamma}$. If each block of $I$ in $\mathcal{G}(R)$ is the subregular representation of $\mathrm{SU}(2)$ in $\mathrm{U}\left(N_{\ell}\right)$, then the moduli space is a product of ALE spaces $M_{\xi_{0}}^{\prime} \times M_{\xi_{1}}^{\prime} \times \cdots \times M_{\xi_{\Gamma_{\Gamma}}}^{\prime}$ where $M_{\xi_{\ell}}^{\prime}$ 
is a minimal resolution of the orbifold $\mathbb{C}^{2} / \mathbb{Z}_{N_{\ell}}$ for $\ell=0,1, \ldots, r_{\Gamma}$; note that $M_{\xi_{\ell}}^{\prime}$ is a point if $N_{\ell}=1$. Due to $\Gamma$-invariance of the frame $\left(\mathrm{d} \tau, \hat{e}^{a}\right)$ and hence of the components of $\underline{A}$, irreducible connections occur only for irreducible actions of $\Gamma$ on the $\mathrm{V}$-bundle $\underline{V_{R}}$, i.e. when $N_{\ell}=N$ for some $\ell \in\left\{0,1, \ldots, r_{\Gamma}\right\}$ and $N_{\ell^{\prime}}=0$ for $\ell^{\prime} \neq \ell$, in which case the moduli space is the four-dimensional hyper-Kähler manifold $M_{\xi_{\ell}}^{\prime} \rightarrow \mathbb{C}^{2} / \mathbb{Z}_{N}$; for example, the four-parameter family of 't Hooft oneinstanton solutions [18, Sect. 5]

$$
Y_{\tau}=0 \quad \text { and } \quad Y_{a}=\frac{1}{s+\lambda} I_{a} \quad \text { with } \quad \lambda \in \mathbb{R}_{\geq 0}
$$

live on the base of this deformation for $\lambda=0$. In dimensions $\geq 8$ with $T_{a}=0$ the moduli space is the cone over the 3-Sasakian homogeneous manifold $\mathrm{SU}(N) / \mathrm{S}(\mathrm{U}(N-2) \times \mathrm{U}(1))$ of dimension $4(N-1)$ with $N \geq 3$.

Sasakian quiver gauge theory. In Sect. 4 we considered the $\mathrm{SU}(2)$-equivariant dimensional reduction of Yang-Mills theory from $M \times S^{3} / \Gamma$ to $M$. For the cones we consider instead the reduction from $\tilde{M} \times C\left(S^{3} / \Gamma\right)$ to $\tilde{M} \times \mathbb{R}_{>0}$, which by the $\Gamma$-action in (3.7) is described by the same quiver (in applications to holography $\tilde{M}$ is the boundary of $M$ ).

The only change in the resulting quiver gauge theory action is the addition of a term proportional to $\operatorname{tr}_{N} \mathcal{F}_{\tau a} \mathcal{F}^{\tau a}$ in (4.9). This additional term allows for a vacuum state of the quiver gauge theory on $\tilde{M}$ with not only flat gauge fields $\mathcal{F}_{a b}=0=\mathcal{F}_{\tau a}$ but also anti-self-dual gauge fields $\mathcal{F}$ on $C\left(S^{3} / \Gamma\right)$. The reduction of the anti-self-dual Yang-Mills equations on the cone $C\left(S^{3} / \Gamma\right)$, with the conformally equivalent metric (2.32), for $\mathrm{SU}(2)$-invariant connections

$$
\mathcal{A}=X_{\tau} \mathrm{d} \tau+X_{a} e^{a}
$$

on the V-bundle $\underline{V_{R}}$ is carried out in [18, Sect. 5] and shown to be given by Nahm-type equations

$$
\frac{\mathrm{d} X_{a}}{\mathrm{~d} \tau}=2 X_{a}-\frac{1}{2} \varepsilon_{a}^{b c}\left[X_{b}, X_{c}\right]-\left[X_{\tau}, X_{a}\right] .
$$

As the one-forms $e^{a}$ are not $\Gamma$-invariant, the matrices $X_{a}$ now decompose as in (3.11), allowing for reducible $\Gamma$-actions on $\underline{V_{R}}$.

These equations have a solution with constant matrices $X_{a}=2 I_{a}$ and $X_{\tau}=0$ which yields vanishing curvature $\mathcal{F}=0$, where $I_{a} \in \mathcal{R e p}_{\mathrm{Q}_{\Gamma}, \mathrm{R}_{\Gamma}}(R) / / \mathrm{PG}(R)$ are $\mathrm{SU}(2)$ generators in the $N$ dimensional irreducible representation on the vector space $V_{R}$; this is in contrast to the case of flat space considered earlier where all such matrices are necessarily diagonal [12]. For the multiplicity space $R=\widehat{V}$ of the regular representation of $\Gamma$, i.e. when $N_{\ell}=n_{\ell}$ for all $\ell=0,1, \ldots, r_{\Gamma}$, the moduli space of all constant matrices is the orbifold $M_{0}=\mathbb{C}^{2} / \Gamma$, as discussed above. We shall find that the moduli space of $\tau$-dependent solutions with suitable boundary conditions at $\tau \rightarrow \pm \infty$ is also an orbifold $M_{0}^{\prime}=\mathbb{C}^{2} / \Gamma^{\prime}$ for $\Gamma^{\prime}=\mathbb{Z}_{|\Gamma|}$, where $\Gamma^{\prime}=\Gamma$ only for the A series; in this instance $I_{a}$ embed the group $\mathrm{SU}(2)$ irreducibly into $\mathrm{PG}=\mathrm{SU}(|\Gamma|)$. This expectation is supported by the explicit BPSTtype instanton solutions $X_{a}=f(\tau) I_{a}, X_{\tau}=0$ constructed by [18, Sect. 5] in this case (see below) which are parameterized by a four-dimensional moduli space consisting of one dilatation parameter valued in $\mathbb{R}_{\geq 0}$ and three gauge rotational $\mathrm{SU}(2)$ parameters, analogously to [32, Sect. 7]; since the subgroup of $\mathrm{SU}(|\Gamma|)$ which commutes with $I_{a}$ in this case is its center $\mathbb{Z}_{|\Gamma|}$, the gauge rotations are actually valued in $\mathrm{SU}(2) / \mathbb{Z}_{|\Gamma|}=S^{3} / \mathbb{Z}_{|\Gamma|}$ and so the moduli space is the cone $C\left(S^{3} / \mathbb{Z}_{|\Gamma|}\right)$.

Sasakian quiver varieties. To substantiate and extend these statements, we generalize the infinite-dimensional hyper-Kähler quotient construction of the moduli space of SU(2)-invariant instantons on $C\left(S^{3}\right)$ (see e.g. [30, Sect. 5]) to the general setting of quiver varieties. We put

$$
C_{1}=-Z_{t}+\mathrm{i} Z_{3}:=\frac{1}{2 t}\left(-X_{\tau}+\mathrm{i} X_{3}\right) \quad \text { and } \quad C_{2}=Z_{1}+\mathrm{i} Z_{2}:=\frac{1}{2 t}\left(X_{1}+\mathrm{i} X_{2}\right)
$$


where $t:=\mathrm{e}^{2 \tau}=r^{2}$. For a $\Gamma$-module (3.2), we may identify the $t$-dependent $\Gamma$-equivariant matrices $C_{1}, C_{2}: \mathbb{R}_{>0} \rightarrow \operatorname{End}_{\mathbb{C}}\left(V_{R}\right)$ with maps $\mathbb{R}_{>0} \rightarrow \mathcal{R e p}_{Q_{\Gamma}^{\text {op }}}(R)$ into the representation space of the opposite quiver associated to the Sasakian quiver $\mathrm{Q}_{\Gamma}$; we denote this infinite-dimensional affine space by $\widehat{\operatorname{Rep}}_{Q_{\Gamma}^{\text {op }}}(R)$. For this, let

$$
\mathbb{C}_{\Gamma}^{2}=\bigoplus_{\ell=0}^{r_{\Gamma}} d_{\ell}^{\Gamma} V_{\ell}
$$

be a two-dimensional representation of the orbifold group $\Gamma$ determined by the $\Gamma$-equivariance conditions on $C_{1}, C_{2}$ described in Sects. 2-3. Let $A^{\Gamma}=\left(a_{\ell \ell^{\prime}}\right)$ be the adjacency matrix of the quiver $\mathrm{Q}_{\Gamma}$, i.e. $a_{\ell \ell^{\prime}}^{\Gamma}$ is the number of arrows joining vertex $\ell$ to vertex $\ell^{\prime}$; note that $A^{\Gamma}$ is not a symmetric matrix in general. Then the multiplicities $d_{\ell}^{\Gamma}$ can be determined by using the tensor product multiplicities $m_{\ell \ell^{\prime}} \ell^{\prime \prime}$ appearing in the Clebsch-Gordan decomposition

$$
V_{\ell} \otimes V_{\ell^{\prime}}=\bigoplus_{\ell^{\prime \prime}=0}^{r_{\Gamma}} m_{\ell \ell^{\prime}} \ell^{\prime \prime} V_{\ell^{\prime \prime}}
$$

via the relations

$$
\sum_{\ell^{\prime \prime}=0}^{r_{\Gamma}} d_{\ell^{\prime \prime}}^{\Gamma} m_{\ell^{\prime \prime} \ell^{\ell^{\prime}}}=a_{\ell \ell^{\prime}}^{\Gamma} \quad \text { for } \quad \ell, \ell^{\prime}=0,1, \ldots, r_{\Gamma} .
$$

Then by analogous arguments to those which led to (5.2), we find

$$
\operatorname{Hom}_{\Gamma}\left(V_{R}, \mathbb{C}_{\Gamma}^{2} \otimes V_{R}\right)=\bigoplus_{\ell, \ell^{\prime}=0}^{r_{\Gamma}} a_{\ell \ell^{\prime}}^{\Gamma} \operatorname{Hom}_{\mathbb{C}}\left(R_{\ell^{\prime}}, R_{\ell}\right)=\mathcal{R e p}_{\mathrm{Q}_{\Gamma}^{\mathrm{op}}}(R)
$$

from which the identification follows.

The space $\widehat{\mathcal{R e p}}_{Q_{\Gamma}^{\text {op }}}(R)$ has the natural structure of an infinite-dimensional quaternionic vector space by identifying the $\Gamma$-module $\mathbb{C}_{\Gamma}^{2}$ as a module of rank one over the quaternions $\mathbb{H}$. With suitable boundary conditions that we describe below, it carries a metric defined by the $\mathrm{L}^{2}$-norm

$$
\left\|\left(c_{1}, c_{2}\right)\right\|^{2}:=\int_{\mathbb{R}_{>0}} \mathrm{~d} t \operatorname{tr}_{N}\left(c_{1}^{\dagger} c_{1}+c_{2}^{\dagger} c_{2}\right)
$$

and a holomorphic symplectic form

$$
\omega_{\mathbb{C}}\left(\left(c_{1}, c_{2}\right),\left(c_{1}^{\prime}, c_{2}^{\prime}\right)\right):=\int_{\mathbb{R}_{>0}} \mathrm{~d} t \operatorname{tr}_{N}\left(c_{1} c_{2}^{\prime}-c_{1}^{\prime} c_{2}\right),
$$

where $c_{i}:=\delta C_{i}$ are solutions of the linearised (around $C_{i}$ given by (6.10)) equations (6.9). Let $\widehat{\mathcal{G}}(R)$ be the group of gauge transformations $g: \mathbb{R}_{>0} \rightarrow \mathcal{G}(R)$ which are trivial at infinity; it acts on $\widehat{\operatorname{Rep}}_{Q_{\Gamma}^{\text {op }}}(R)$ as

$$
C_{1} \longmapsto g C_{1} g^{-1}-\frac{1}{2} \frac{\mathrm{d} g}{\mathrm{~d} t} g^{-1} \quad \text { and } \quad C_{2} \longmapsto g C_{2} g^{-1}
$$

These ingredients endow $\widehat{\operatorname{Rep}}_{Q_{\Gamma}^{\text {op }}}(R)$ with the structure of a flat hyper-Kähler Banach manifold which is invariant under the action of $\widehat{\mathcal{G}}(R)$. The corresponding $(1,1)$-form is

$$
\omega_{\mathbb{R}}\left(\left(c_{1}, c_{2}\right),\left(c_{1}^{\prime}, c_{2}^{\prime}\right)\right):=\frac{1}{2} \int_{\mathbb{R}_{>0}} \mathrm{~d} t \operatorname{tr}_{N}\left(c_{1} c_{1}^{\dagger}-c_{1}^{\prime} c_{1}^{\dagger}+c_{2} c_{2}^{\prime \dagger}-c_{2}^{\prime} c_{2}^{\dagger}\right)
$$


With $\widehat{\mathfrak{u}}(N)$ denoting the Lie algebra of infinitesimal gauge transformations $\mathbb{R}_{>0} \rightarrow \mathfrak{u}(N)$ which are trivial at infinity, the corresponding hyper-Kähler moment map $\mu=\left(\mu_{\mathbb{C}}, \mu_{\mathbb{R}}\right): \widehat{\mathcal{R e p}}_{Q_{\Gamma}^{\text {op }}}(R) \rightarrow$ $\mathbb{R}^{3} \otimes \widehat{\mathfrak{u}}(N)$ is given by

$$
\begin{aligned}
& \mu_{\mathbb{C}}\left(C_{1}, C_{2}\right)=\frac{\mathrm{d} C_{2}}{\mathrm{~d} t}+\left[C_{1}, C_{2}\right] \\
& \mu_{\mathbb{R}}\left(C_{1}, C_{2}\right)=\frac{\mathrm{i}}{2}\left(\frac{\mathrm{d} C_{1}}{\mathrm{~d} t}+\frac{\mathrm{d} C_{1}^{\dagger}}{\mathrm{d} t}+\left[C_{1}, C_{1}^{\dagger}\right]+\left[C_{2}, C_{2}^{\dagger}\right]\right) .
\end{aligned}
$$

The vanishing locus $\mu^{-1}(0)$ then coincides with the solution space of the Nahm equations (6.9). Notice how this moment map formally compares with that of the McKay quiver variety from Sect. 5 by setting $W_{4}=\frac{\mathrm{d}}{\mathrm{d} t}+Z_{t}$ and $W_{a}=Z_{a}$ for $a=1,2,3$. Its image belongs to the set of gauge equivalence classes of elements valued in the subspace $\mathbb{R}^{3} \otimes \widehat{\mathcal{R e p}}_{Q_{\Gamma}^{\text {op }}}(R) \subset \mathbb{R}^{3} \otimes \widehat{\mathfrak{u}}(N)$ : Via a gauge transformation (6.17) one can go to a temporal gauge with $Z_{t}=0$ in which the components of the moment map are given by

$$
\mu_{a}(Z)=\frac{\mathrm{d} Z_{a}}{\mathrm{~d} t}+\frac{1}{2} \varepsilon_{a}^{b c}\left[Z_{b}, Z_{c}\right]
$$

for $a=1,2,3$.

However, in contrast to the moment maps used in the construction of Nakajima quiver varieties, here $\mu$ is not $\Gamma$-invariant. Hence our vacuum moduli are generically parameterized by an orbifold $\mu^{-1}(0) / \mathrm{P} \widehat{\mathcal{G}}(R)$ which cannot be described as a hyper-Kähler quotient. As long as the action of the Lie group $\mathrm{P} \widehat{\mathcal{G}}(R)$ is proper, the space of orbits $\mu^{-1}(0) / \mathrm{P} \widehat{\mathcal{G}}(R)$ has the structure of a stratified Hausdorff differential space (see e.g. [35]). Here we shall take an explicit and illuminating route that avoids the intricate technical stacky issues involved in taking such quotients: We first describe the hyper-Kähler quotient corresponding to the full unbroken gauge group $\mathcal{G}=\mathrm{U}(N)$, and then implement $\Gamma$-equivariance; we denote the (singular) quotient space obtained in this way by $\widehat{\operatorname{Rep}}_{\mathrm{Q}_{\Gamma}^{\mathrm{op}}}(R) / / \mathrm{P} \widehat{\mathcal{G}}(R)$.

It remains to specify suitable boundary conditions for the equations $\mu_{\mathbb{C}}=\mu_{\mathbb{R}}=0$. Using results of [36], one can put solutions of the Nahm equations into a Coulomb gauge such that $C_{1}, C_{2}$ converge as $t \rightarrow \infty$ to the exact "model" solution

$$
\tilde{C}_{1}=\frac{\mathrm{i}}{2} T_{3}+\frac{\mathrm{i}}{t+\lambda} J_{3} \quad \text { and } \quad \tilde{C}_{2}=\frac{1}{2} T_{+}+\frac{1}{t+\lambda} J_{+}
$$

where $\lambda \in \mathbb{R}_{\geq 0}$ is a scale parameter, the elements $T_{3}, T_{+}=T_{1}+\mathrm{i} T_{2}$ are valued in the Cartan subalgebra of the Lie algebra (5.6), and $J_{3}, J_{+}=J_{1}+$ i $J_{2}$ generate a representation of $\mathrm{SU}(2)$ in $\mathrm{U}(N)$ which resides in the representation space (6.14) and which commutes with $T_{a}$, i.e. $J_{a}$ for $a=1,2,3$ take values in the Lie algebra of the common centralizer of $T_{a}$ in $\mathrm{U}(N)$. Suitable moduli spaces of Nahm data asymptotic to this model solution yield complex coadjoint orbits of $\mathrm{U}(N)[15,16,36]$. The model solution with $T_{a}=0$ is exactly the BPST-type instanton solution on the orbifold $\mathbb{R}^{4} / \Gamma$ which is discussed in $[18$, Sect. 5]; it generates nilpotent orbits [15]. On the other hand, if the joint centralizer of $T_{a}$ is the maximal torus $\mathrm{U}(1)^{N}$, then $J_{a}=0$ and the orbits are regular [16]. Henceforth we set $J_{a}=0$ but keep $T_{a}$ arbitrary corresponding to generic semisimple orbits.

Thus analogously to the boundary conditions in (6.5), we consider solutions with the asymptotics

$$
\lim _{t \rightarrow \infty} C_{1}(t)=\frac{\mathrm{i}}{2} T_{3} \quad \text { and } \quad \lim _{t \rightarrow \infty} C_{2}(t)=\frac{1}{2} T_{+},
$$

and which acquire simple poles

$$
\lim _{t \rightarrow 0} t C_{1}(t)=\frac{\mathrm{i}}{2} I_{3} \quad \text { and } \quad \lim _{t \rightarrow 0} t C_{2}(t)=\frac{1}{2} I_{+}
$$


at $t=0$ with residues defining a representation $I_{3}, I_{+}=I_{1}+\mathrm{i} I_{2}$ of $\mathfrak{s u}(2)$ in (6.14). We denote by $\widehat{\mathcal{R e p}}_{\mathrm{Q}_{\Gamma}^{\mathrm{op}}}(R)_{\xi, I}$ the subspace of $\widehat{\mathcal{R e p}}_{\mathrm{Q}_{\Gamma}^{\text {op }}}(R)$ consisting of pairs $\left(C_{1}, C_{2}\right)$ satisfying these boundary conditions and with suitable analytic behaviour, and by $\widehat{\mathcal{G}}(R)_{I} \subset \widehat{\mathcal{G}}(R)$ the subgroup of gauge transformations preserving the boundary conditions (6.24). Here $\xi=\left(\xi_{\ell}^{a}\right)$ with $\xi_{\ell}^{a} \in \mathbb{R}$ for $a=1,2,3$ and $\ell=0,1, \ldots, r_{\Gamma}$ parameterize the periods of the trisymplectic structure determined by $T_{a}$, while fixing the singular part of the Nahm data to $I=\left(I_{a}\right)$ guarantees that tangent vectors (infinitesimal deformations) are regular and square-integrable so that the $\mathrm{L}^{2}$-metric (6.15) is well-defined; these boundary conditions are the main distinguishing feature from the construction of moduli spaces of (framed) monopoles. In terms of our original SU(2)-invariant instantons which are parameterized by the connections (6.8), the boundary conditions (6.23)-(6.24) mean that they have regular values at the origin which determine an $\mathrm{SU}(2)$ representation $I=\left(I_{a}\right)$ in $\mathrm{U}(N)$, whereas their behaviour at infinity is governed by a pole at $r=\infty$ with residue which gives the Fayet-Iliopoulos parameters of the quiver gauge theory; this somewhat undesirable asymptotic behaviour will be eliminated by our construction below.

The corresponding quiver variety is then the infinite quotient

$$
\mathcal{M}_{\xi, I}\left(\mathrm{Q}_{\Gamma}, R\right):=\widehat{\operatorname{Rep}}_{\mathrm{Q}_{\Gamma}^{\mathrm{op}}}(R)_{\xi, I} / / \mathrm{P} \widehat{\mathcal{G}}(R)_{I} .
$$

This quotient space is finite-dimensional: In the temporal gauge the moment map equations $\mu_{a}=0$ from (6.21) can be written as

$$
\left[Z_{a}, Z_{b}\right]+\varepsilon_{a b}^{c} \frac{\mathrm{d} Z_{c}}{\mathrm{~d} t}=0
$$

for $a, b=1,2,3$; this leaves the space of solutions to the ordinary differential equations (6.26) modulo the action of the finite-dimensional gauge group (3.9). Putting $Z_{a}=\frac{1}{2 t} X_{a}$ for constant $X_{a}$ sets up a one-to-one correspondence between such solutions of the equations (6.26) and solutions of the BPS equations (4.10) describing the vacuum moduli space of quiver gauge theory based on the Sasakian quiver with relations $\left(Q_{\Gamma}, R_{\Gamma}\right)$.

Orbits and slices. Let us now describe the Higgs branch of vacuum states $\mathcal{M}_{\xi, I}\left(\mathrm{Q}_{\Gamma}, R\right)$ more explicitly. By a standard symplectic quotient argument [17], the hyper-Kähler quotient by $\mathrm{P} \widehat{\mathcal{G}}_{I}$ is equivalent to the holomorphic symplectic quotient $\mu_{\mathbb{C}}^{-1}(0) / \mathrm{P} \widehat{\mathcal{G}}_{I}^{\mathbb{C}}$ by the action (6.17) of the complexification $\widehat{\mathcal{G}}_{I}^{\mathbb{C}}$ of the gauge group $\widehat{\mathcal{G}}_{I}$. The complex Nahm equation $\mu_{\mathbb{C}}\left(C_{1}, C_{2}\right)=0$ implies that the path $C_{2}(t)$ lies in the same adjoint orbit in the complex Lie algebra $\mathfrak{g}^{\mathbb{C}}:=\mathfrak{g} \otimes \mathbb{C}$ for all $t \in \mathbb{R}_{>0}$. It also implies that the Casimir invariants of $C_{2}$ are independent of $t$. Since we quotient only by gauge transformations which are trivial at infinity, the boundary condition (6.23) then implies that the Casimir invariants of $C_{2}$ coincide with those of $T_{+}$.

It follows that the moduli space of solutions to the Nahm equations with the boundary conditions (6.23) is the closure $\overline{\mathcal{O}_{T_{+}}}$of the adjoint orbit $\mathcal{O}_{T_{+}}$of $T_{+}$in $\mathfrak{g}^{\mathbb{C}}$ obtained by adding the (finitely many) orbits of elements that have the same Casimir invariants as $T_{+}$. The orbit $\mathcal{O}_{T_{+}}$is a complex symplectic manifold, with the standard Kirillov-Kostant-Souriau symplectic form, of dimension $\operatorname{dim}_{\mathbb{C}} \mathcal{O}_{T_{+}}=\operatorname{dim}_{\mathbb{C}} \mathcal{G}^{\mathbb{C}}-\operatorname{dim}_{\mathbb{C}} \mathcal{Z}_{T_{+}}$where $\mathcal{Z}_{T_{+}} \subset \mathcal{G}^{\mathbb{C}}$ is the subgroup that commutes with $T_{+}$; note that $\mathcal{T}^{\mathbb{C}} \subseteq \mathcal{Z}_{T_{+}}$. If $T_{+}$is regular, i.e. $\operatorname{dim}_{\mathbb{C}} \mathcal{Z}_{T_{+}}=\operatorname{dim}_{\mathbb{C}} \mathcal{T}^{\mathbb{C}}$, then $\overline{\mathcal{O}_{T_{+}}}=\mathcal{O}_{T_{+}}$as every element of $\mathfrak{g}^{\mathbb{C}}$ with the same Casimir invariants is conjugate to $T_{+}$in this case; in general, the closure $\overline{\mathcal{O}_{T_{+}}}$ always contains a regular orbit.

Next we have to implement the correct pole structure (6.24) at $t=0$ which is determined by a representation $I$ of $\mathrm{SU}(2)$ in $\mathcal{G}$; representations of $\mathrm{SU}(2)$ in $\mathrm{U}(N)$ are in one-to-one correspondence with ordered partitions $\vec{\lambda}=\left(\lambda_{1}, \ldots, \lambda_{s}\right)$ of $N$ with at most $N$ parts, which correspond 
combinatorially to Young diagrams with $N$ boxes and at most $N$ rows. By definition they satisfy

$$
\sum_{i=1}^{s} \lambda_{i}=N \quad \text { with } \quad \lambda_{1} \geq \lambda_{2} \geq \cdots \geq \lambda_{s}>0
$$

where the part $\lambda_{i}$ is the dimension of the $i$-th irreducible $\mathrm{SU}(2)$-module occuring in the decomposition of the fundamental representation $\mathbb{C}^{N}$ of $\mathrm{U}(N)$ as a representation of $\mathrm{SU}(2)$; the integer $s:=\ell(\vec{\lambda})$ is called the length of the partition $\vec{\lambda}$. Solutions of the complex Nahm equation with this singular behaviour are in a bijective correspondence with points in the Slodowy slice [15] which is the affine subspace of $\mathfrak{g}^{\mathbb{C}}$ given by

$$
\mathcal{S}_{I}=I_{+}+\mathfrak{z}\left(I_{-}\right),
$$

where $\mathfrak{z}\left(I_{-}\right)$is the centralizer of $I_{-}$in $\mathfrak{g}^{\mathbb{C}}$; the correspondence associates to points of $(6.28)$ the solution of the complex Nahm equation given by

$$
C_{1}(t)=\frac{\mathrm{i}}{2 t} I_{3} \quad \text { and } \quad C_{2}(t)=\frac{1}{2 t} I_{+}+\sum_{\alpha \in P_{-}} t^{-m_{\alpha}} c_{\alpha} v_{\alpha},
$$

where we used a complex gauge transformation to gauge fix $C_{1}$; here $c_{\alpha} \in \mathbb{C}$ and $P_{-}$parameterizes the lowest weight vectors $v_{\alpha} \in \mathfrak{g}^{\mathbb{C}}$ of weight $m_{\alpha} \in \frac{1}{2} \mathbb{Z}_{<0}$ for the adjoint action of $\mathrm{SU}(2)$ on $\mathfrak{g}^{\mathbb{C}}$, i.e. $\left[I_{-}, v_{\alpha}\right]=0$ and $\left[\mathrm{i} I_{3}, v_{\alpha}\right]=m_{\alpha} v_{\alpha}$, so that $\sum_{\alpha \in P_{-}} c_{\alpha} v_{\alpha} \in \mathfrak{z}\left(I_{-}\right)$. The Slodowy slice $\mathcal{S}_{I}$ intersects $\mathcal{O}_{I_{+}}$in the single point $I_{+}$transversally, i.e. $\mathcal{S}_{I} \oplus T_{I_{+}} \mathcal{O}_{I_{+}}=\mathfrak{g}^{\mathbb{C}}$, and it meets only those orbits whose closures contain $\mathcal{O}_{I_{+}}$where it has transverse intersections which are thereby submanifolds of $\mathfrak{g}^{\mathbb{C}}$.

It follows that the moduli space of solutions to the Nahm equations with boundary conditions (6.23)-(6.24) is the intersection $\overline{\mathcal{O}_{T_{+}}} \cap \mathcal{S}_{I}$ of dimension $\operatorname{dim}_{\mathbb{C}} \mathcal{Z}_{I_{-}}-\operatorname{dim}_{\mathbb{C}} \mathcal{T}^{\mathbb{C}}$; it is a complex symplectic manifold with the restriction of the Kirillov-Kostant-Souriau symplectic form. Here the dimension of the centralizer $\mathcal{Z}_{I_{-}}$of $I_{-}$coincides with the number of summands in the decomposition of $\mathfrak{g}^{\mathbb{C}}$ into irreducible representations of $\mathrm{SU}(2)$, as each irreducible representation has a one-dimensional subspace of lowest weight vectors; as $\mathrm{SU}(2)$-modules are self-dual, we can explicitly decompose $\mathfrak{g}^{\mathbb{C}} \cong \mathbb{C}^{N} \otimes \mathbb{C}^{N}$ under the $\mathrm{SU}(2)$ embedding $I$ by using the fact that for each positive integer $n=2 j+1$ with $j \in \frac{1}{2} \mathbb{Z}_{\geq 0}$ the Lie group $\mathrm{SU}(2)$ has a unique irreducible spin- $j$ representation on $\mathbb{C}^{n}$ which obey the Clebsch-Gordan rules

$$
\mathbb{C}^{n} \otimes \mathbb{C}^{n^{\prime}} \cong \bigoplus_{j^{\prime \prime}=\left|j-j^{\prime}\right|}^{j+j^{\prime}} \mathbb{C}^{n^{\prime \prime}}
$$

with $n^{\prime}=2 j^{\prime}+1$ and $n^{\prime \prime}=2 j^{\prime \prime}+1$. It is shown by [17] that this manifold can be naturally identified with the hyper-Kähler quotient $\overline{\mathcal{O}_{T_{+}}} \cap \mathcal{S}_{I} \cong\left(\overline{\mathcal{O}_{T_{+}}} \times \mathrm{PG} \mathcal{G}^{\mathbb{C}} \times \mathcal{S}_{I}\right) / / /_{0}$ PG which is interpreted as matching the two solutions to the Nahm equations coming from $\overline{\mathcal{O}_{T_{+}}}$on $\mathbb{R}_{>0}$ and from $\mathrm{PG} \mathcal{G}^{\mathbb{C}} \times \mathcal{S}_{I}$ on $(0,1]$; the latter moduli space consists of pairs $\left(g(1), C_{2}\right)$, where $g:(0,1] \rightarrow \mathrm{PG}^{\mathbb{C}}$ is the unique complex gauge transformation which gauge fixes $C_{1}$ in (6.29).

It remains to implement $\Gamma$-equivariance. This requires that the pair $\left(C_{1}, C_{2}\right)$ belong to the representation space (6.14), and hence our moduli space (6.25) can be described easily as the intersection

$$
\mathcal{M}_{\xi, I}\left(\mathrm{Q}_{\Gamma}, R\right) \cong \overline{\mathcal{O}_{T_{+}}} \cap \mathcal{S}_{I} \cap \operatorname{Rep}_{\mathrm{Q}_{\Gamma}^{\mathrm{op}}}(R)
$$

which is naturally a hyper-Kähler variety with the restricted hyper-Kähler structure of $\overline{\mathcal{O}_{T_{+}}} \cap \mathcal{S}_{I}$. Since the complex gauge transformation which fixes $C_{1}$ in $(6.29)$ resides in $\mathrm{PG}(R)^{\mathbb{C}}$, and with the understanding that the $\mathrm{SU}(2)$ representation $I$ already resides in (6.14) (or else (6.31) is empty), 
by restricting the gauge group $\mathcal{G}$ to the subgroup $\mathcal{G}(R) \subset \mathrm{U}(N)$ we can compute the dimension of the moduli space of vacua using the hyper-Kähler quotient construction above to get

$$
\begin{aligned}
\operatorname{dim}_{\mathbb{R}} \mathcal{M}_{\xi, I}\left(\mathrm{Q}_{\Gamma}, R\right)= & \operatorname{dim}_{\mathbb{R}}\left(\left(\overline{\mathcal{O}_{T_{+}}} \cap \mathcal{R e p}_{\mathrm{Q}_{\Gamma}^{\mathrm{op}}}(R)\right) \times \mathrm{PG}(R)^{\mathbb{C}} \times\left(\mathcal{S}_{I} \cap \mathcal{R e p}_{\mathrm{Q}_{\Gamma}^{\mathrm{op}}}(R)\right)\right) \\
& -4 \operatorname{dim}_{\mathbb{R}} \mathrm{P} \mathcal{G}(R) \\
= & \operatorname{dim}_{\mathbb{R}} \operatorname{Rep}_{\mathrm{Q}_{\Gamma}^{\text {op }}}(R)-2 \operatorname{dim}_{\mathbb{R}} \mathcal{T}(R)+\operatorname{dim}_{\mathbb{R}}\left(\mathfrak{z}\left(I_{-}\right) \cap \operatorname{Rep}_{\mathrm{Q}_{\Gamma}^{\text {op }}}(R)\right) \\
& -2 \operatorname{dim}_{\mathbb{R}} \mathcal{G}(R) \\
= & \operatorname{dim}_{\mathbb{R}}\left(\mathfrak{z}\left(I_{-}\right) \cap \mathcal{R e p}_{\mathrm{Q}_{\Gamma}^{\text {op }}}(R)\right)+2 \vec{N} \cdot\left(A^{\Gamma}\right)^{\top} \vec{N}-2 \sum_{\ell=0}^{r_{\Gamma}} N_{\ell}\left(N_{\ell}+1\right) .
\end{aligned}
$$

For Sasakian quivers this dimension is always a multiple of four.

Nilpotent cones. On imposing $\Gamma$-equivariance, one generically encounters further phenomena. The $\Gamma$-equivariance of $C_{2}$ generally requires $T_{+}=0$ and therefore the Casimir invariants of $T_{+}$all vanish; the corresponding spherically symmetric instanton solutions are then regular at $r=\infty$. The only elements $C_{2}$ of $\mathfrak{g}^{\mathbb{C}}$ which have vanishing Casimir elements are nilpotent elements. For $\mathfrak{g}=\mathfrak{u}(N)$, one can conjugate any $N \times N$ complex matrix to its Jordan normal form which for a traceless nilpotent matrix takes a block diagonal form $\boldsymbol{J}_{+}=\operatorname{diag}\left(\boldsymbol{J}_{d_{1}}, \ldots, \boldsymbol{J}_{d_{m}}\right)$ where $\boldsymbol{J}_{d_{p}}$ for $p=1, \ldots, m$ is a regular nilpotent $d_{p} \times d_{p}$ matrix of the form

$$
\boldsymbol{J}_{d_{p}}=\left(\begin{array}{ccccc}
0 & 1 & 0 & \cdots & 0 \\
0 & 0 & 1 & \ddots & \vdots \\
\vdots & \vdots & \ddots & \ddots & 0 \\
0 & 0 & \cdots & 0 & 1 \\
0 & 0 & \cdots & 0 & 0
\end{array}\right)
$$

with $\left(\boldsymbol{J}_{d_{p}}\right)^{d_{p}}=0$, whose centralizer is generated by $\boldsymbol{J}_{d_{p}},\left(\boldsymbol{J}_{d_{p}}\right)^{2}, \ldots,\left(\boldsymbol{J}_{d_{p}}\right)^{d_{p}-1}$; here we have decomposed $N=\sum_{p=1}^{m} d_{p}$ into integers satisfying $d_{1} \geq d_{2} \geq \cdots \geq d_{m}>0$. Note that $\vec{d}=\left(d_{1}, \ldots, d_{m}\right)$ defines an ordered partition of $N$ and hence corresponds to an embedding of $\mathrm{SU}(2)$ in $\mathrm{U}(N)$ with nilpotent generator $\boldsymbol{J}_{+}$; that every nilpotent element arises in this way is a consequence of the Jacobson-Morozov theorem.

In particular, there is a unique regular nilpotent element $\boldsymbol{J}_{N}$, corresponding to the irreducible representation of $\mathrm{SU}(2)$ on $\mathbb{C}^{N}$ with partition $\vec{d}=(N)$, which has vanishing Casimir invariants and which generates the regular nilpotent orbit $\mathcal{O}_{J_{N}}$ of maximal dimension. The closure of this orbit is the nilpotent cone $\mathcal{N}=\overline{\mathcal{O}_{J_{N}}}$, of dimension $\operatorname{dim}_{\mathbb{C}} \mathcal{N}=\operatorname{dim}_{\mathbb{C}} \mathcal{G}^{\mathbb{C}}-\operatorname{dim}_{\mathbb{C}} \mathcal{T}^{\mathbb{C}}$, consisting of all nilpotent elements of $\mathfrak{g}^{\mathbb{C}}$, each generating finitely many orbits; the irreducible subvariety $\mathcal{N}$ has singularities corresponding to non-regular nilpotent orbits. Among these orbits there is the unique subregular nilpotent orbit whose closure contains all non-regular nilpotent orbits and has complex codimension two in $\mathcal{N}$; it corresponds to the subregular representation of $\mathrm{SU}(2)$ in $\mathrm{U}(N)$ with $\vec{d}=(N-1,1)$ and appears as the locus of Kleinian quotient singularities $\mathbb{C}^{2} / \mathbb{Z}_{N}$ in $\mathcal{N}$ [37] (the cone over $\left.S^{3} / \mathbb{Z}_{N}\right)$. On the other hand, the element $T_{+}=0$ generates the unique nilpotent orbit consisting of a single singular point in $\mathcal{N}$. The minimal nilpotent orbit is the unique nilpotent orbit of smallest non-zero dimension, which is generated by the highest root vector of $\mathfrak{g}^{\mathbb{C}}$ and thereby consists of $N \times N$ matrices $H$ of rank one with $H^{2}=0$; it corresponds to the SU(2) embedding with $\vec{d}=(2,1,1, \ldots, 1)$ and is the cone over $\mathrm{SU}(N) / \mathrm{S}(\mathrm{U}(N-2) \times \mathrm{U}(1))$ of complex dimension $2(N-1)$.

It follows that our moduli space (6.31) in this case is the singular variety

$$
\mathcal{M}_{0, I}\left(\mathrm{Q}_{\Gamma}, R\right) \cong \mathcal{N} \cap \mathcal{S}_{I} \cap \operatorname{Rep}_{\mathrm{Q}_{\Gamma}^{\text {op }}}(R)
$$


of dimension given by the formula (6.32); the appearence of orbifold singularities here is not surprising given our earlier observation concerning the stacky nature of the quotient parameterizing the vacuum moduli. The structure of the singular locus and the dimension of the moduli space now depend on the embedding $I$ of $\mathfrak{s u}(2)$ in $\operatorname{Rep}_{Q_{\Gamma}^{\text {op }}}(R)$ that determines the transverse slice $\mathcal{S}_{I}$ to the orbit of the nilpotent element $I_{+}$, and on the particular Sasakian quiver $Q_{\Gamma}$. In general, our moduli spaces generically have higher dimension than the naive (non-equivariant) prediction because of two non-standard features: The representation varieties $\operatorname{Rep}_{\mathrm{Q}_{\Gamma}^{\text {op }}}(R) \subset \mathfrak{u}(N)$ contain the Lie algebras (5.6) as proper subalgebras, while the quotient defining the moduli space is taken with respect to the broken gauge group (3.9) which is a proper subgroup of $\mathrm{U}(N)$. For example, if $I=0$ is the trivial representation corresponding to the partition $\vec{\lambda}=(1,1, \ldots, 1)$, then $\mathcal{S}_{0}=\mathfrak{g}^{\mathbb{C}}$ and hence the moduli space is simply the cone

$$
\mathcal{M}_{0,0}\left(\mathrm{Q}_{\Gamma}, R\right) \cong \mathcal{N} \cap \operatorname{Rep}_{\mathrm{Q}_{\Gamma}^{\mathrm{op}}}(R)
$$

consisting of all $\Gamma$-equivariant nilpotent endomorphisms of $V_{R}$; it has dimension

$$
\operatorname{dim}_{\mathbb{R}} \mathcal{M}_{0,0}\left(\mathrm{Q}_{\Gamma}, R\right)=4 \vec{N} \cdot\left(A^{\Gamma}\right)^{\top} \vec{N}-2 \sum_{\ell=0}^{r_{\Gamma}} N_{\ell}\left(N_{\ell}+1\right) .
$$

$\boldsymbol{A}_{\boldsymbol{k}}$. For the cyclic group $\Gamma=\mathbb{Z}_{k+1}$ with $k=2 q$, the adjacency matrix of the quiver (3.25) is given by $a_{\ell \ell^{\prime}}^{\Gamma}=\delta_{\ell^{\prime} \ell}+\delta_{\ell^{\prime}, \ell+1}$ and the tensor product multiplicities are $m_{\ell \ell^{\prime}} \ell^{\ell^{\prime \prime}}=\delta_{\ell^{\prime \prime}, \ell+\ell^{\prime}}$. By (6.13) the two-dimensional $\Gamma$-module (6.11) decomposes into irreducible representations as $\mathbb{C}_{\Gamma}^{2}=V_{0} \oplus V_{1}$ so that

$$
\mathbb{C}_{\Gamma}^{2} \otimes V_{\ell}=V_{\ell} \oplus V_{\ell+1}
$$

for $\ell=0,1, \ldots, k$; note that $\mathbb{C}_{\Gamma}^{2}$ is not a self-dual representation of $\Gamma$. With $C_{\mathrm{Q}_{\Gamma}}=2 \mathbf{1}_{\mathbb{C}^{k+1}}-A^{\Gamma}-$ $\left(A^{\Gamma}\right)^{\top}$ the generalized Cartan matrix of the Sasakian quiver $\mathrm{Q}_{\Gamma}$, by (5.24) the dimension of the corresponding Nakajima quiver variety is given by

$$
\operatorname{dim}_{\mathbb{R}} \mathcal{X}_{\xi}\left(Q_{\Gamma}, R\right)=4+4 \sum_{\ell=0}^{k} N_{\ell} N_{\ell+1} .
$$

It follows that all representations $R$ of the Sasakian quiver in this case are indecomposable and correspond to imaginary roots; the Nakajima quiver variety is four-dimensional for irreducible quiver bundles (4.2) corresponding to simple representations $R$ with $N_{\ell}=N$ for some $\ell \in\left\{0,1, \ldots, r_{\Gamma}\right\}$ and $N_{\ell^{\prime}}=0$ for $\ell^{\prime} \neq \ell$.

The respective summands in (6.37) correspond to the matrix pairs $\left(C_{1}, C_{2}\right)$ which can be decomposed analogously to (4.12)-(4.13) as

$$
C_{1}=\operatorname{diag}\left(\rho_{0}, \rho_{1}, \ldots, \rho_{k}\right) \quad \text { and } \quad C_{2}=\left(\begin{array}{ccccc}
0 & \phi_{1} & 0 & \cdots & 0 \\
0 & 0 & \phi_{2} & \ddots & \vdots \\
\vdots & \vdots & \ddots & \ddots & 0 \\
0 & 0 & \cdots & 0 & \phi_{k} \\
\phi_{k+1} & 0 & \cdots & 0 & 0
\end{array}\right) \text {, }
$$

with $\rho_{\ell}: \mathbb{R}_{>0} \rightarrow \operatorname{End}_{\mathbb{C}}\left(R_{\ell}\right)$ and $\phi_{\ell+1}: \mathbb{R}_{>0} \rightarrow \operatorname{Hom}_{\mathbb{C}}\left(R_{\ell+1}, R_{\ell}\right)$ for $\ell=0,1, \ldots, k$. The complex Nahm equations determined by the complex moment map $\mu_{\mathbb{C}}$ from (6.19) read as

$$
\frac{\mathrm{d} \phi_{\ell+1}}{\mathrm{~d} t}=\phi_{\ell+1} \rho_{\ell+1}-\rho_{\ell} \phi_{\ell+1}
$$


in $\operatorname{Hom}_{\mathbb{C}}\left(R_{\ell+1}, R_{\ell}\right)$ for $t \in \mathbb{R}_{>0}$ and $\ell=0,1, \ldots, k$, while the real Nahm equations from $\mu_{\mathbb{R}}$ in $(6.20)$ are given by

$$
\frac{\mathrm{d} \rho_{\ell}}{\mathrm{d} t}+\frac{\mathrm{d} \rho_{\ell}^{\dagger}}{\mathrm{d} t}=\phi_{\ell+1} \phi_{\ell+1}^{\dagger}-\phi_{\ell}^{\dagger} \phi_{\ell}-\left[\rho_{\ell}, \rho_{\ell}^{\dagger}\right]
$$

in $\operatorname{End}_{\mathbb{C}}\left(R_{\ell}\right)$ for $t \in \mathbb{R}_{>0}$ and $\ell=0,1, \ldots, k$; here the boundary conditions (6.23) require setting $T_{+}=0$. Note the formal similarily between these equations and the constant F-term and D-term relations (4.26) and (4.27): In the temporal gauge $Z_{t}=0$ the path $\rho_{\ell}$ is Hermitian, and the right-hand sides of (4.26) and (4.27) are replaced with the corresponding radial variations in (6.40) and (6.41).

By (6.32) the dimension of the corresponding Sasakian quiver variety is given by

$$
\operatorname{dim}_{\mathbb{R}} \mathcal{M}_{0, I}\left(\mathrm{Q}_{\Gamma}, R\right)=\operatorname{dim}_{\mathbb{R}}\left(\mathfrak{z}\left(I_{-}\right) \cap \mathcal{R e p}_{\mathrm{Q}_{\Gamma}^{\mathrm{op}}}(R)\right)+2 \sum_{\ell=0}^{k} N_{\ell}\left(N_{\ell+1}-1\right) .
$$

In particular, when $N_{\ell}=1$ for all $\ell=0,1, \ldots, k$ (so that $N=k+1$ ) this dimension formula becomes

$$
\operatorname{dim}_{\mathbb{R}} \mathcal{M}_{0, I}\left(\mathrm{Q}_{\Gamma}, \widehat{V}\right)=\operatorname{dim}_{\mathbb{R}}\left(\mathfrak{z}\left(I_{-}\right) \cap \operatorname{Rep}_{\mathrm{Q}_{\Gamma}^{\text {op }}}(\widehat{V})\right),
$$

whereas the real dimension of the nilpotent cone $\mathcal{N}$ in $\mathfrak{s l}(k+1, \mathbb{C})$ is $2 k(k+1)$. Let us examine some particular cases for illustration.

For the trivial representation $I=0$, the moduli space has dimension $4(k+1) \geq 8$ and it contains the BPST-type instanton solutions from [18, Sect. 5], i.e. the model solution (6.22) with $T_{a}=0$, $\lambda>0$ and $J_{a}$ the generators of the regular embedding of the group $\mathrm{SU}(2)$ into $\mathrm{SU}(k+1)$; this four-parameter family lies in the subcone $C\left(S^{3} / \mathbb{Z}_{k+1}\right)$ along the subregular orbit of the equivariant nilpotent cone $\mathcal{M}_{0,0}\left(\mathrm{Q}_{\Gamma}, \widehat{V}\right)=\mathcal{N} \cap \operatorname{Rep}_{\mathrm{Q}_{\Gamma}^{\text {op }}}(\widehat{V})$ in the complex Lie algebra $\mathfrak{s l}(k+1, \mathbb{C})$.

Now let us consider the regular representation $I=I^{\text {reg }}$ of $\mathrm{SU}(2)$ in $\mathrm{SU}(k+1)$. To determine the centralizer $\mathfrak{z}\left(I_{-}\right)$in $\operatorname{Rep}_{\mathrm{Q}_{\Gamma}^{\text {op }}}(\widehat{V})$ in this case, we need to determine the space of matrices $(6.39)$ which commute with the nilpotent matrix $\boldsymbol{J}_{k+1}$ from (6.33). It is easy to see that the general form of such matrices is given by $C_{1}=\rho \mathbf{1}_{\mathbb{C}^{k+1}}$ and $C_{2}=\phi \boldsymbol{J}_{k+1}$ for arbitrary $\rho, \phi \in \mathbb{C}$; hence the moduli space in this case is four-dimensional. Upon intersecting with the nilpotent cone $\mathcal{N}$ in $\mathfrak{s l}(k+1, \mathbb{C})$, we expect to see the singular locus $\mathbb{C}^{2} / \mathbb{Z}_{k+1}$ by Brieskorn's theorem [37]. This can be checked directly: It remains to quotient the space $(\rho, \phi) \in \mathbb{C}^{2}$ by the commutant subgroup of $\mathrm{SU}(2)$ in $\mathrm{SU}(k+1)$, which in this case is simply the center $\mathbb{Z}_{k+1}$ of $\mathrm{SU}(k+1)$ and hence the moduli space is biholomorphic to the orbifold singularity

$$
\mathcal{M}_{0, I^{\mathrm{reg}}}\left(\mathrm{Q}_{\Gamma}, \widehat{V}\right) \cong C\left(S^{3} / \mathbb{Z}_{k+1}\right)
$$

This is in marked contrast to the non-equivariant case where the moduli space would consist of just the single element $I_{+}$. The model solution (6.22), with $T_{a}=0, \lambda=0$ and $J_{a}$ the generators of the regular embedding of $\mathrm{SU}(2)$, lives in this moduli space. Recall that this was precisely the situation for the solution (6.7); in this sense the 't Hooft and BPST instantons are "equivalent" for fixed $\mathrm{PG}(R)=\mathrm{SU}(N)$. Moreover, for the minimal charge instantons and suitable boundary conditions, by taking a trivial $\Gamma$-action one can extend instantons from $\mathbb{R}^{4}$ to $\mathbb{R}^{4} / \Gamma$ and its deformation $M_{\xi}$, giving the same four-dimensional moduli space. On the other hand, by an appropriate choice of non-trivial $\Gamma$-action on the rank $N$ vector bundle $E \rightarrow M_{\xi}$ and suitable embedding of $\mathrm{SU}(2)$ in $\mathrm{SU}(N)$ at infinity, one obtains BPST-type instantons on $\mathbb{C}^{2} / \Gamma$ in a four-dimensional moduli space via both ADHM and Nahm equations.

A completely analogous calculation shows that for the subregular representation $I=I^{\text {subreg }}$ the dimension of the moduli space is equal to 8, and upon dividing by the commutant subgroup the 
moduli space can be described topologically as the cone

$$
\mathcal{M}_{0, I \text { subreg }}\left(\mathrm{Q}_{\Gamma}, \widehat{V}\right) \cong C(\mathrm{SU}(3) / \mathrm{S}(\mathrm{U}(1) \times \mathrm{U}(1))) .
$$

One can carry on with smaller representations of $\mathrm{SU}(2)$ whose centralizers have complex dimension two or more; in general the commutant subgroup of $\mathrm{SU}(k+1)$ is a Lie group of rank $\ell(\vec{\lambda})-1$, where $\vec{\lambda}$ is the partition corresponding to the $\mathrm{SU}(2)$ embedding $I$, and it is abelian if and only if the parts of $\vec{\lambda}$ are all distinct integers. The construction continues until we reach the trivial representation $I=0$ with maximal moduli space dimension $4(k+1)$. When some $N_{\ell}>1$ one encounters moduli spaces of even higher dimensions.

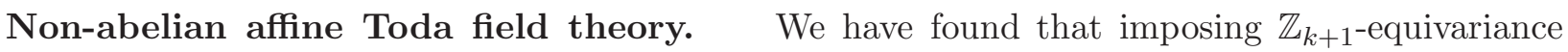
on the matrix pairs $\left(C_{1}, C_{2}\right)$ yields matrices (6.39) and reduces the anti-self-duality equations on $\mathbb{C}^{2} / \mathbb{Z}_{k+1}$ to the equations (6.40)-(6.41). With $N_{\ell}=1$ for all $\ell=0,1, \ldots, k$, the equation (6.40) can be written as

$$
\frac{\mathrm{d} \log \phi_{\ell+1}}{\mathrm{~d} t}=\rho_{\ell+1}-\rho_{\ell}
$$

By taking $\rho_{\ell}, \phi_{\ell} \in \mathbb{R}$ (where Hermiticity of $C_{1}$ is automatic in the temporal gauge $Z_{t}=0$ ), differentiating (6.46) with respect to $t$ and using (6.41) we get the equations

$$
2 \frac{\mathrm{d}^{2} \log \phi_{\ell+1}}{\mathrm{~d} t^{2}}=\left(\phi_{\ell+2}\right)^{2}-2\left(\phi_{\ell+1}\right)^{2}+\left(\phi_{\ell}\right)^{2}
$$

which are the equations of the affine Toda lattice associated with the $\widehat{A}_{k}$-type Lie algebra for rotationally symmetric fields in two-dimensions, see e.g. [38, App. A]. These equations are explicitly integrable and their solutions are parameterized in terms of $2(k+1)$ arbitrary constants, where the integrability is based on the underlying group theory structure and the solutions can be expressed as particular matrix elements in the fundamental representation of $\mathrm{SU}(k+1)$. Imposing the requirements that these solutions vanish as $t \rightarrow \infty$ and that they admit the appropriate pole structure (6.24) at $t=0$ reduces the number of free parameters accordingly. For example, the $2(k+1)$-parameter family of solutions presented in [38, eqs. (A31)-(A33)] vanish at $t=\infty$ and are regular at the origin $t=0$; for generic values of these parameters the solutions correspond to the trivial representation $I=0$ of $\mathrm{SU}(2)$. Solutions with residues at $t=0$ defining non-trivial representations of $\mathrm{SU}(2)$ in $\mathrm{SU}(k+1)$ require fixing some of these parameters appropriately. All of this agrees with our analysis of the moduli space of solutions above for the $\widehat{A}_{k}$-type quiver gauge theory.

For $N_{\ell}>1$, our equations (6.40)-(6.41) are a variant of non-abelian affine Toda lattice equations; however, they do not coincide exactly with the existing non-abelian generalizations considered previously in the literature, see e.g. [39]. Nevertheless, our equations have a Lax pair and zero curvature presentation from their origin as anti-self-duality equations in four dimensions, and in the abelian limit they coincide with the affine Toda lattice equations; hence we may refer to (6.40)-(6.41) as non-abelian affine Toda lattice equations. We also have an explicit description of their moduli spaces of solutions as real slices of the (singular) hyper-Kähler moduli spaces of our Sasakian quiver gauge theories. This description may have important uses as generalizations of the standard conformal two-dimensional $\widehat{A}_{k}$ Toda field theories which are well studied in the literature. In particular, our approach is reminescent of the recent AGT duality [40, 41] which relates them to four-dimensional $\mathcal{N}=2$ superconformal quiver gauge theories of $\widehat{A}_{k}$-type.

$\boldsymbol{A}_{n}$ quiver gauge theory. In the non-equivariant case $\Gamma=\{1\}$, Kronheimer's moduli spaces of $\mathrm{SU}(2)$-invariant $\mathrm{U}(N)$-instantons on $C\left(S^{3}\right)$ with $T_{a}=0$ can be regarded in certain cases as 
particular classes of Nakajima quiver varieties associated to a linear $A_{n}$ quiver (4.28) for some $n \leq N$ determined by the $\mathrm{SU}(2)$ embedding $I$ [29, Sect. 8]; the identification is based on the ADHM transform of instantons on $\mathbb{R}^{4}$. The ADHM moduli space is itself a quiver variety based on the Jordan quiver

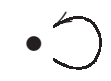

which is the oriented graph of the $\widehat{A}_{0}$ Dynkin diagram corresponding to the $k=0$ limit of the cyclic group $\mathbb{Z}_{k+1}$; the representation space of the corresponding double quiver is $\operatorname{Hom}_{\mathbb{C}}\left(W_{Q}, \mathbb{C}^{2} \otimes W_{Q}\right)$ where $W_{Q} \cong \mathbb{C}^{N}$. The Kronheimer moduli space is constructed from the $\mathrm{SU}(2)$-invariant part of $\operatorname{Hom}_{\mathbb{C}}\left(W_{Q}, \mathbb{C}^{2} \otimes W_{Q}\right)$, in much the same way that the representation space (5.3) parameterizes $\Gamma$-equivariant instantons on $\mathbb{R}^{4}$. Now we decompose the vector space $W_{Q}$ into irreducible representations of $\mathrm{SU}(2)$ on $\mathbb{C}^{\ell}$ as

$$
W_{Q}=\bigoplus_{\ell=1}^{n} Q_{\ell} \otimes \mathbb{C}^{\ell} \quad \text { with } \quad Q_{\ell} \cong \mathbb{C}^{v_{\ell}}
$$

where the dimension vector $\vec{v}=\left(v_{1}, \ldots, v_{n}\right)$ represents the $\mathrm{SU}(2)$-module structure of the instantons at the origin. From the Clebsch-Gordan decomposition (6.30) we have $\mathbb{C}^{2} \otimes \mathbb{C}^{\ell}=\mathbb{C}^{\ell-1} \oplus \mathbb{C}^{\ell+1}$, and hence by Schur's lemma $\operatorname{Hom}_{\mathrm{SU}(2)}\left(W_{Q}, \mathbb{C}^{2} \otimes W_{Q}\right)$ coincides with the representation variety of the double of the $A_{n}$ quiver (4.28). To accomodate a non-trivial holonomy of the anti-self-dual connection at infinity which is specified by an $\mathrm{SU}(2)$ embedding $I$ corresponding to an ordered partition $\vec{\lambda}$ of $N$, we consider the corresponding framed quiver with framing nodes specifying a representation $W_{S}$ of $\mathrm{SU}(2)$ in $\mathrm{U}(N)$ with $S_{\ell} \cong \mathbb{C}^{w_{\ell}}$, where the dimension vector $\vec{w}=\left(w_{1}, \ldots, w_{n}\right)$ labels the number $0 \leq w_{\ell} \leq N$ of parts of $\vec{\lambda}$ with $\lambda_{i}=\ell$ for $\ell=1, \ldots, n$; then

$$
N=\sum_{\ell=1}^{n} \ell w_{\ell} \quad \text { and } \quad \ell(\vec{\lambda})=\sum_{\ell=1}^{n} w_{\ell} .
$$

We denote this framed Nakajima quiver variety by $\mathcal{X}_{0}\left(\mathrm{Q}_{A_{n}} ; Q, S\right) \cong \mathcal{N} \cap \mathcal{S}_{I}$; the dimension vectors $\vec{v}$ and $\vec{w}$ obey certain consistency relations with the nilpotent orbits in $\mathcal{N}$ which are described in $[29$, Sect. 8].

In the $\Gamma$-equivariant case, we have to take the intersection (6.34). Moreover the roles of the vector spaces $W_{Q}$ and $W_{S}$ are interchanged: The $\mathrm{SU}(2)$-module $W_{Q}$ now describes the behaviour of the instanton connection at infinity, while $W_{S}$ describes the $\mathrm{SU}(2)$-module structure of its regular value at the origin $r=0$. Demanding as usual that the $\mathrm{SU}(2)$ representation fit into the $\Gamma$ equivariant structure, it follows that our moduli space admits a presentation as the subvariety

$$
\mathcal{M}_{0, I}\left(\mathrm{Q}_{\Gamma}, R\right) \cong \mathcal{X}_{0}\left(\mathrm{Q}_{A_{n}} ; Q, S\right) \cap \mathcal{R e p}_{\mathrm{Q}_{\Gamma}^{\mathrm{op}}}(R)
$$

of $\Gamma$-equivariant maps in a framed Nakajima quiver variety associated to a linear $A_{n}$ quiver. This gives a realization of the vacuum states of the Sasakian quiver gauge theory in the Higgs moduli spaces of certain $A_{n}$ quiver gauge theories.

Because of the role reversal of boundary conditions, we cannot interpret this presentation as an ADHM matrix model of the type which arises from systems of $\mathrm{D} p-\mathrm{D}(p+4)$ branes. Instead, as the scalar fields $Z_{a}$ for $a=1,2,3$ have a Nahm pole boundary condition parameterized by the partition $\vec{\lambda}$, we may give an (indirect) interpretation of our moduli of Nahm data in terms of configurations of $\mathrm{D} p-\mathrm{D}(p+2)-\mathrm{D}(p+4)$ branes following [19]. For this, we consider a system of $N$ parallel $\mathrm{D}(p+2)$-branes wrapping $C\left(S^{3} / \Gamma\right)$ which transversally intersect $\ell(\vec{\lambda}) \mathrm{D}(p+4)$-branes with $\lambda_{i} \mathrm{D}(p+2)$-branes ending on the $i$-th $\mathrm{D}(p+4)$-brane at the apex $r=0$ of the cone $C\left(S^{3} / \Gamma\right)$ for $i=1, \ldots, \ell(\vec{\lambda})$. The $\mathrm{D}(p+2)$-branes support a four-dimensional $\mathcal{N}=2$ quiver gauge theory based on the $A_{n}$ quiver, with scalar fields $Z_{a}$ for $a=1,2,3$ along the $\mathrm{D}(p+4)$-branes such that 
$\left(C_{1}, C_{2}\right) \in \operatorname{Hom}_{\mathrm{SU}(2)}\left(W_{Q}, \mathbb{C}^{2} \otimes W_{Q}\right)$; the gauge group at the $\ell$-th node of the quiver $(4.28)$ is $\mathrm{U}\left(w_{\ell}\right)$ for $\ell=1, \ldots, n$. Due to the orbifold singularity at $r=0$, there are an additional $N_{\ell}$ constituent fractional $\mathrm{D} p$-branes probing the $\mathrm{D}(p+4)$-branes. It would be interesting to give a more direct picture in terms of the original scalar fields $X_{a}$ from the $\mathrm{SU}(2)$-equivariant dimensional reduction over the cone $C\left(S^{3} / \Gamma\right)$.

\section{Acknowledgments}

The work of OL and ADP was partially supported by the Deutsche Forschungsgemeinschaft under Grant LE 838/13. The work of RJS was partially supported by the Consolidated Grant ST/L000334/1 from the UK Science and Technology Facilities Council. This work was completed while RJS was visiting the Hausdorff Research Institute for Mathematics in Bonn during the 2014 Trimester Program "Noncommutative Geometry and its Applications"; he would like to thank Alan Carey, Victor Gayral, Matthias Lesch, Walter van Suijlekom and Raimar Wulkenhaar for the invitation, and all the staff at HIM for the warm hospitality.

\section{References}

[1] L. Alvarez-Cónsul and O. García-Prada, "Dimensional reduction and quiver bundles," J. Reine Angew. Math. 556 (2003) 1 [arXiv:math-dg/0112160].

[2] O. Lechtenfeld, A.D. Popov and R.J. Szabo, "Quiver gauge theory and noncommutative vortices," Prog. Theor. Phys. Suppl. 171 (2007) 258 [arXiv:0706.0979 [hep-th]].

[3] B.P. Dolan and R.J. Szabo, "Equivariant dimensional reduction and quiver gauge theories," Gen. Rel. Grav. 43 (2010) 2453 [arXiv:1001.2429 [hep-th]].

[4] M.R. Douglas and G.W. Moore, "D-branes, quivers, and ALE instantons," arXiv:hepth/9603167.

[5] C.V. Johnson and R.C. Myers, "Aspects of type IIB theory on ALE spaces," Phys. Rev. D 55 (1997) 6382 [arXiv:hep-th/9610140].

[6] M.R. Douglas, B.R. Greene and D.R. Morrison, "Orbifold resolution by D-branes," Nucl. Phys. B 506 (1997) 84 [hep-th/9704151].

[7] S.S. Gubser, N.A. Nekrasov and S.L. Shatashvili, "Generalized conifolds and 4-dimensional $\mathcal{N}=1$ superconformal field theory," JHEP 9905 (1999) 003 [arXiv:hep-th/9811230].

[8] M.R. Douglas, B. Fiol and C. Romelsberger, "The spectrum of BPS branes on a noncompact Calabi-Yau," JHEP 0509 (2005) 057 [arXiv:hep-th/0003263].

[9] A.D. Popov and R.J. Szabo, "Quiver gauge theory of non-abelian vortices and noncommutative instantons in higher dimensions," J. Math. Phys. 47 (2006) 012306 [arXiv:hep-th/0504025].

[10] B.P. Dolan and R.J. Szabo, "Dimensional reduction, monopoles and dynamical symmetry breaking," JHEP 0903 (2009) 059 [arXiv:0901.2491 [hep-th]].

[11] R.J. Szabo and O. Valdivia, "Covariant quiver gauge theories," JHEP 1406 (2014) 144 [arXiv:1404.4319 [hep-th]].

[12] P.B. Kronheimer, "The construction of ALE spaces as hyper-Kähler quotients," J. Diff. Geom. 29 (1989) 665.

[13] P.B. Kronheimer, "A Torelli-type theorem for gravitational instantons," J. Diff. Geom. 29 (1989) 685.

[14] P.B. Kronheimer and H. Nakajima, "Yang-Mills instantons on ALE gravitational instantons," Math. Ann. 288 (1990) 263. 
[15] P.B. Kronheimer, "Instantons and the geometry of the nilpotent variety," J. Diff. Geom. 32 (1990) 473.

[16] P.B. Kronheimer, "A hyper-Kählerian structure on coadjoint orbits of a semisimple complex group," J. London Math. Soc. 42 (1990) 193.

[17] R. Bielawski, "Hyper-Kähler structures and group actions," J. London Math. Soc. 55 (1997) 400; "On the hyper-Kähler metrics associated to singularities of nilpotent varieties," Ann. Global Anal. Geom. 14 (1996) 177.

[18] T.A. Ivanova, O. Lechtenfeld, A.D. Popov and R.J. Szabo, "Orbifold instantons, moment maps and Yang-Mills theory with sources," Phys. Rev. D 88 (2013) 105026 [arXiv:1310.3028 [hep-th]].

[19] D. Gaiotto and E. Witten, "Supersymmetric boundary conditions in $\mathcal{N}=4$ super Yang-Mills theory," J. Statist. Phys. 135 (2009) 789 [arXiv:0804.2902 [hep-th]]; "S-duality of boundary conditions in $\mathcal{N}=4$ super Yang-Mills theory," Adv. Theor. Math. Phys. 13 (2009) 721 [arXiv:0807.3720 [hep-th]].

[20] C.P. Boyer and K. Galicki, Sasakian Geometry (Oxford University Press, 2008).

[21] A.D. Popov and R.J. Szabo, "Double quiver gauge theory and nearly Kähler flux compactifications," JHEP 1202 (2012) 033 [arXiv:1009.3208 [hep-th]].

[22] L. Alvarez-Cónsul and O. García-Prada, "Dimensional reduction, SL(2, C)-equivariant bundles and stable holomorphic chains," Int. J. Math. 12 (2001) 159.

[23] H. Lin and J.M. Maldacena, "Fivebranes from gauge theory," Phys. Rev. D 74 (2006) 084014 [arXiv:hep-th/0509235].

[24] M. Reid, "McKay correspondence," Proc. Alg. Geom. Symp. (Kinosaki) (1996) 14 [arXiv:alggeom/9702016].

[25] M. Cirafici and R.J. Szabo, "Curve counting, instantons and McKay correspondences," J. Geom. Phys. 72 (2013) 54 [arXiv:1209.1486 [hep-th]].

[26] S.K. Donaldson, "Anti-self-dual Yang-Mills connections over complex algebraic surfaces and stable vector bundles," Proc. London Math. Soc. 50 (1985) 1.

[27] K. Uhlenbeck and S.-T. Yau, "On the existence of Hermitian Yang-Mills connections in stable vector bundles," Commun. Pure Appl. Math. 39 (1986) 257.

[28] H. Cassens and P. Slodowy, "On Kleinian singularities and quivers," Prog. Math. 162 (1996) 263.

[29] H. Nakajima, "Instantons on ALE spaces, quiver varieties and Kac-Moody algebras," Duke Math. J. 76 (1994) 365.

[30] N.J. Hitchin, "Hyper-Kähler manifolds," Astérisque 206 (1992) 137.

[31] V.G. Kac, "Infinite root systems, representations of graphs and invariant theory," Invent. Math. 56 (1980) 57.

[32] M. Bianchi, F. Fucito, G. Rossi and M. Martellini, "Explicit construction of Yang-Mills instantons on ALE spaces," Nucl. Phys. B 473 (1996) 367 [arXiv:hep-th/9601162].

[33] H. Nakajima, "Moduli spaces of anti-self-dual connections on ALE gravitational instantons," Invent. Math. 102 (1990) 267.

[34] P. Slodowy, Simple Singularities and Simple Algebraic Groups (Springer, 1980).

[35] J. Śniatycki, Differential Geometry of Singular Spaces and Reduction of Symmetries (Cambridge University Press, 2013).

[36] O. Biquard, "Sur les équations de Nahm et la structure de Poisson des algèbres de Lie semisimples complexes," Math. Ann. 304 (1996) 253. 
[37] E. Brieskorn, "Singular elements of semisimple algebraic groups," Actes Congrés Intern. Math. 2 (1970) 279.

[38] G.V. Dunne, R. Jackiw, S.-Y. Pi and C.A. Trugenberger, "Self-dual Chern-Simons solitons and two-dimensional nonlinear equations," Phys. Rev. D 43 (1991) 1332.

[39] M. Gekhtman, "Hamiltonian structure of non-abelian Toda lattice," Lett. Math. Phys. 46 (1998) 189.

[40] L.F. Alday, D. Gaiotto and Y. Tachikawa, "Liouville correlation functions from fourdimensional gauge theories," Lett. Math. Phys. 91 (2010) 167 [arXiv:0906.3219 [hep-th]].

[41] N. Wyllard, " $A_{N-1}$ conformal Toda field theory correlation functions from conformal $\mathcal{N}=2$ $\mathrm{SU}(N)$ quiver gauge theories," JHEP 0911 (2009) 002 [arXiv:0907.2189 [hep-th]]. 